Aus der Abteilung Rechtsmedizin

(Prof. Dr. med. W. Grellner)

der Medizinischen Fakultät der Universität Göttingen

\title{
Zur Phänomenologie des Obduktionsguts der Rechtsmedizin Göttingen 1969 - 1978 und 1998 - 2007
}

\author{
INAUGURAL-DISSERTATION \\ zur Erlangung des Doktorgrades \\ der Medizinischen Fakultät der \\ Georg-August-Universität zu Göttingen \\ vorgelegt von \\ Babak Rostamzadeh \\ aus Essen
}

Göttingen 2015 
Dekan:

1. Berichterstatter:

2. Berichterstatter:

3. Berichterstatterin:
Prof. Dr. rer. nat. H. K. Kroemer

Prof. Dr. med. Wolfgang Grellner

Prof. Dr. med. Philipp Ströbel

Prof. Dr. hum. biol. Margarete Schön

Tag der mündlichen Prüfung: 09.11.2016 


\section{Inhaltsverzeichnis}

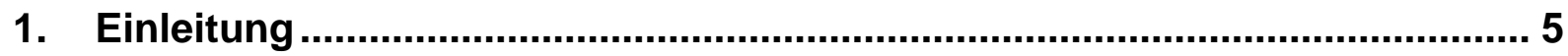

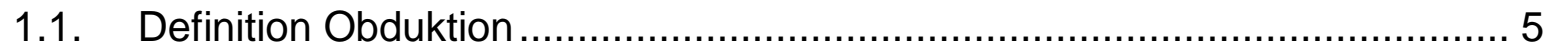

1.2. Bedeutung und Aufgabe der Obduktion ..................................... 5

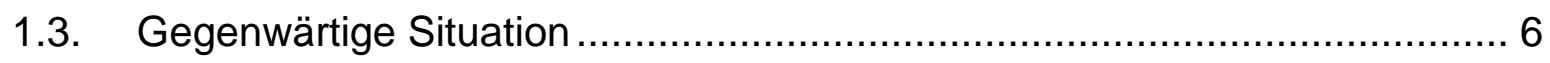

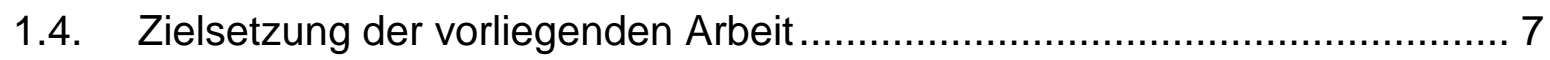

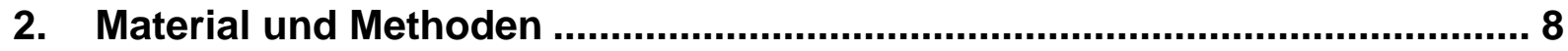

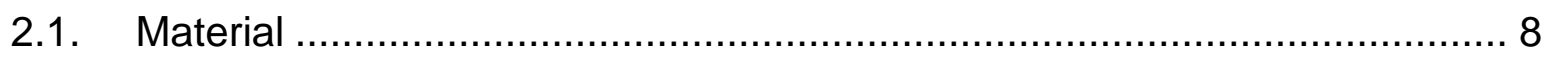

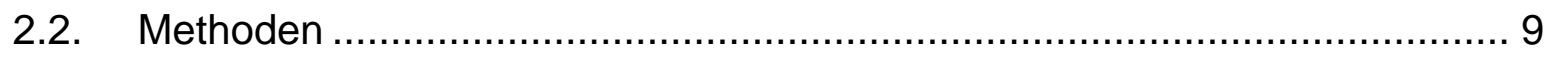

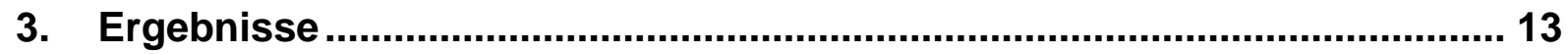

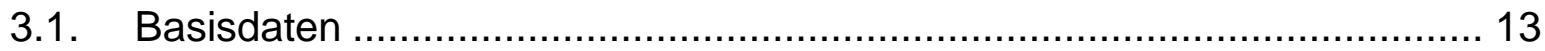

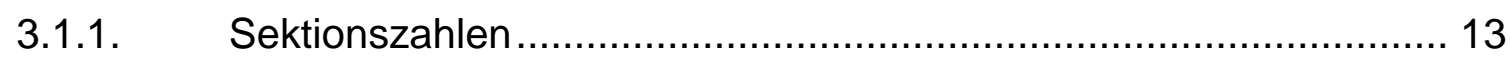

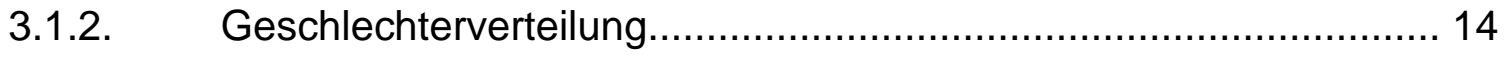

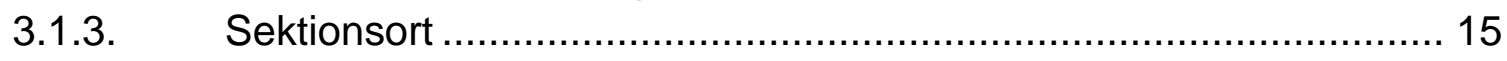

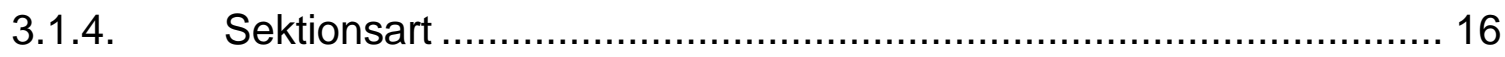

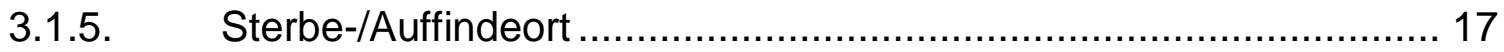

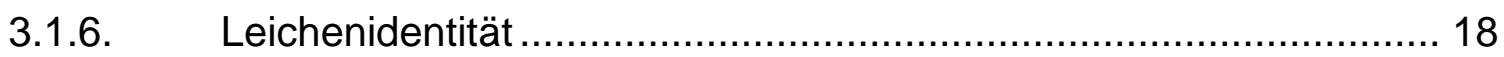

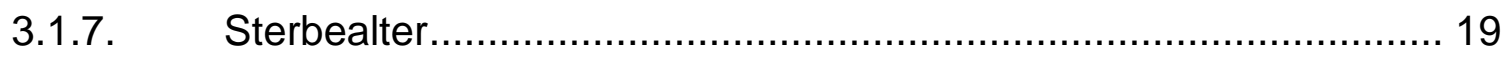

3.1.8. Intervall vom Auffindezeitpunkt bis zur Obduktion...................... 20

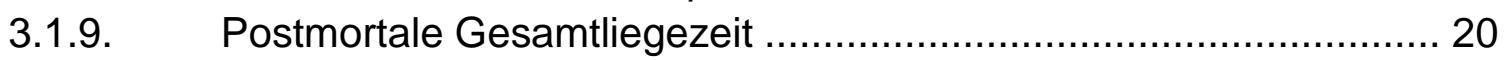

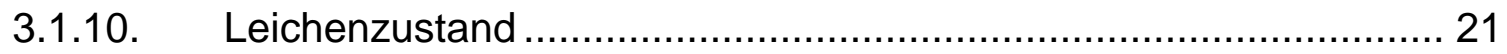

3.1.11. Todesart nach Obduktion .................................................... 22

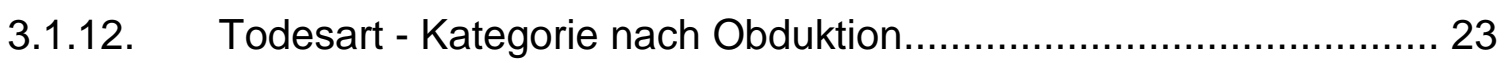

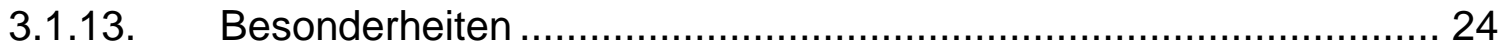

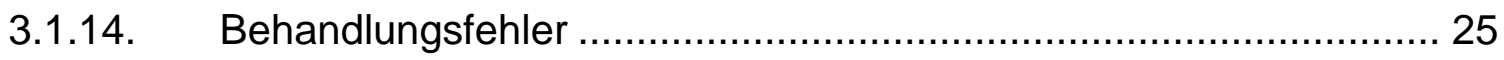

3.1.15. Evidenzgrad der Todesursache......................................... 26

3.1.16. Sterbenstyp bei natürlichem Tod ......................................... 27

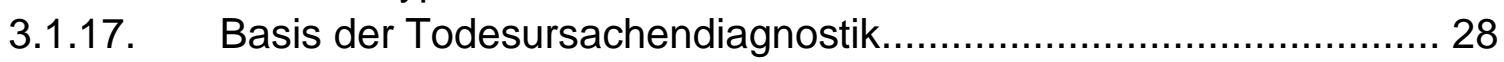

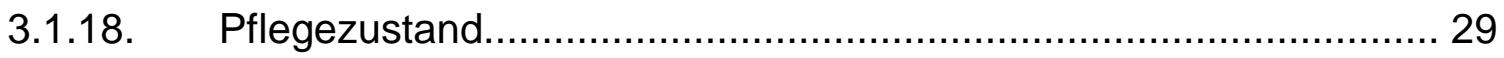

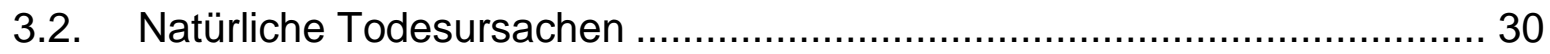

3.3. Nicht natürliche Todesursachen/-umstände .................................. 32

3.3.1. Formen der Gewalteinwirkung ......................................... 32

3.3.2. Anatomische Lokalisation bei stumpfer Gewalteinwirkung............. 33

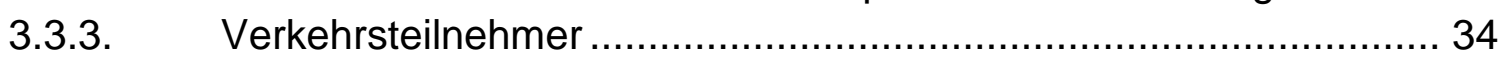

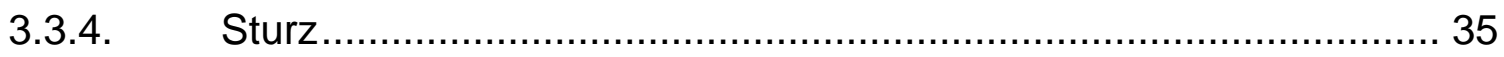

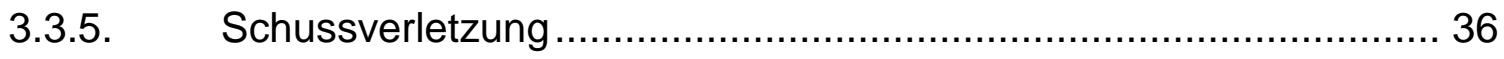

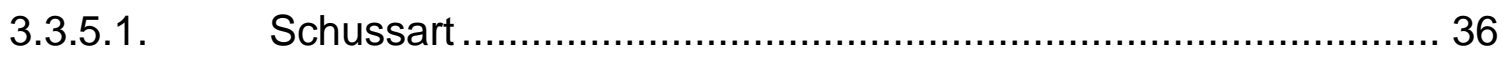


3.3.5.2. Tatwaffe (Schuss- bzw. Explosionseinwirkung) ...................... 37

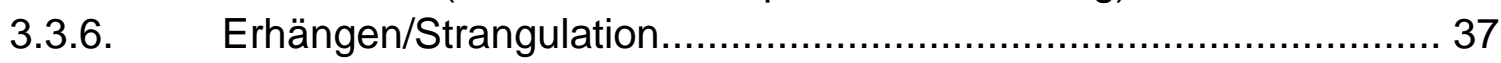

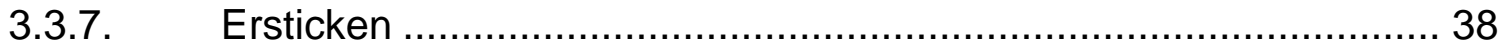

3.3.8. Ertrinken/Tod im Wasser ........................................... 38

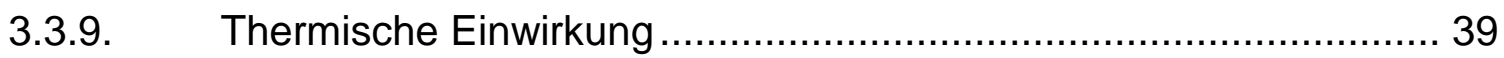

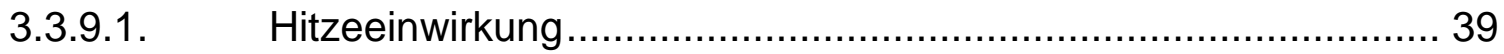

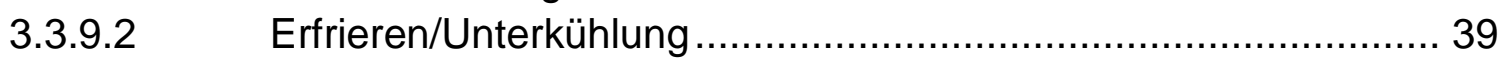

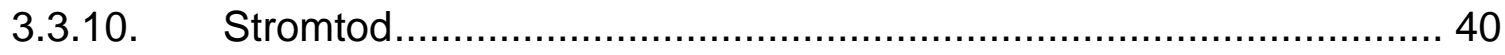

3.3.11. Sonstige Einwirkungen ..................................................... 40

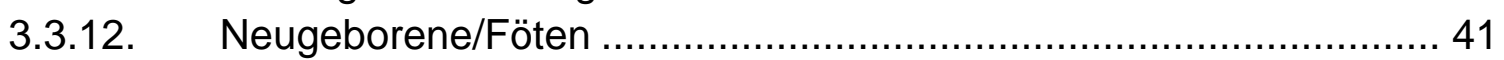

3.3.13. Tod nach medizinischen Maßnahmen.................................... 42

3.3.14. Systemische bzw. direkte Todesursachen .................................. 43

3.3.15. Mittelbare Todesursachen ...................................................... 44

3.3.16. Tötungsdelikte - Gewalteinwirkungen.................................. 45

3.3.17. Intoxikationen ...................................................................... 46

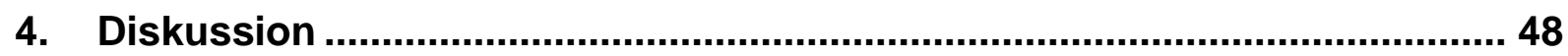

4.1. Diskussion der Methode .......................................................... 48

4.2. Allgemeine Betrachtung zum Obduktionsgut .................................. 48

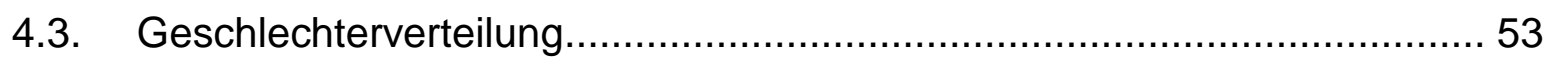

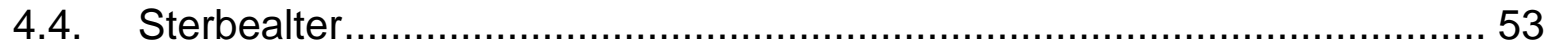

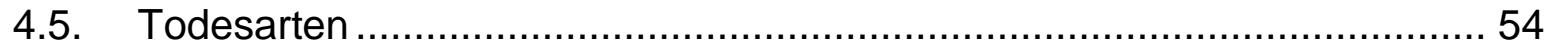

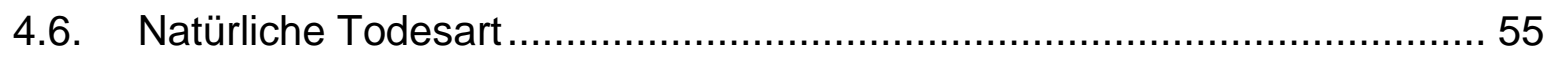

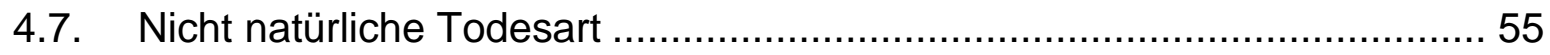

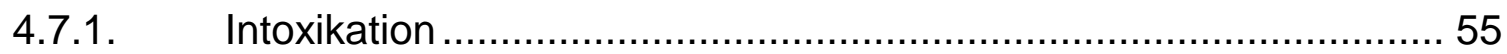

4.7.2. Unfälle (Arbeitsunfälle/Verkehrsunfälle) ................................ 56

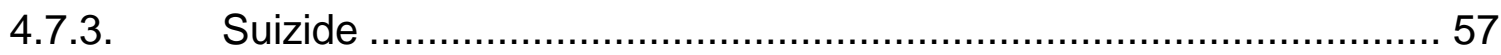

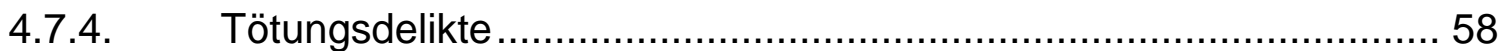

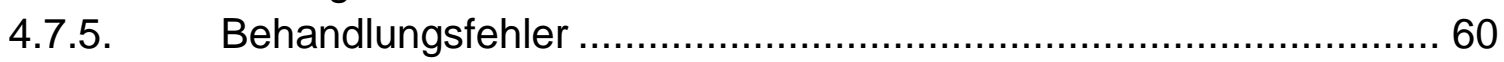

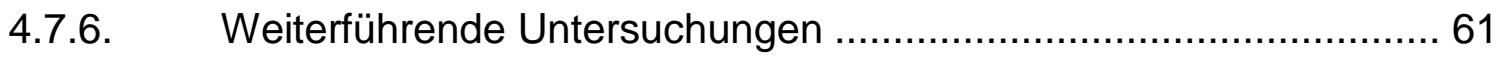

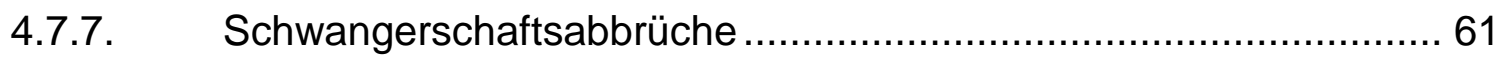

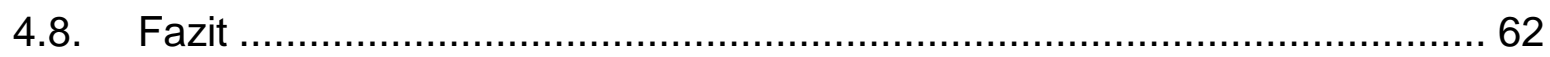

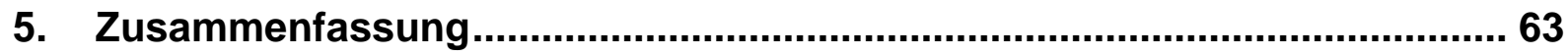

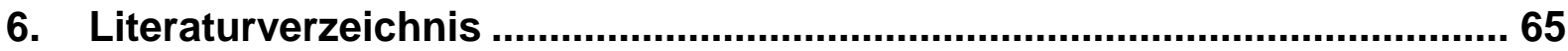

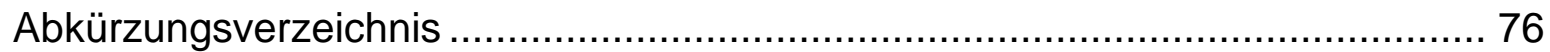

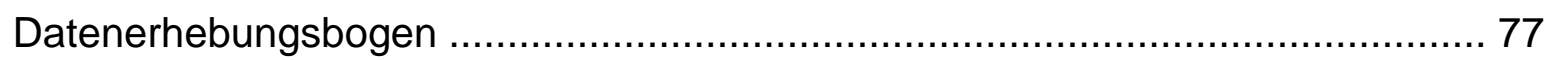




\section{Einleitung}

\subsection{Definition Obduktion}

Unter dem Begriff Obduktion versteht man die innere Leichenschau, die der postmortalen Feststellung von Todesursachen und Grunderkrankungen dient. Man unterscheidet grundsätzlich zwischen klinisch-pathologischen, rechtsmedizinischen und anatomischen Obduktionen.

\subsection{Bedeutung und Aufgabe der Obduktion}

Die Obduktion liefert unerlässliche Informationen zur Krankheitsursache, zum Krankheitsverlauf und zur Todesursache. Weiterhin kann sie als Instrument der Qualitätssicherung zur Überprüfung vorangegangener ärztlicher und pflegerischer Maßnahmen dienen (Brinkmann et al. 2002). Eine weitere wichtige Funktion ist die Validierung der Todesursachenstatistik (Nestler et al. 2008), deren Grundlage Informationen aus dem Leichenschauschein sind. Die Todesursachenstatistik spiegelt das Sterbegeschehen innerhalb einer Gesellschaft wider und bildet die Basis für gesundheitspolitische und -ökonomische Entscheidungen. Um zuverlässige Daten bereitzustellen, sollte eine ausreichend hohe Sektionsfrequenz (25 \% - $35 \%$ ) vorliegen (Schwarze 2001, Madea et al. 2007). Die Sektionsfrequenz (Autopsierate bzw. Obduktionsquote) innerhalb eines bestimmten Zeitraumes wird nach folgender Formel berechnet:

$$
\text { Sektionsfrequenz (\%): } \quad \frac{\text { Anzahl Obduktionen }}{\text { Anzahl Verstorbener }} \times 100
$$

Ferner beinhaltet die rechtsmedizinische Obduktion die „Sicherung von Spuren (an Kleidung und Körper), Körperflüssigkeiten (z. B. Blut, Urin oder Mageninhalt), Sachbeweisen (z.B. Projektilen oder Fremdkörpern), die Feststellung innerer und äußerer Identitätsmerkmale, die Schätzung der Todeszeit, die Beurteilung des Kausalzusammenhangs zwischen Schädigung und dem Tod sowie die Rekonstruktion eines Tathergangs bzw. eines Unfalls“ (Wirth und Strauch 2006, 
S. 58-59). Aufgrund rechtsmedizinischer Obduktionsergebnisse konnten präventive Maßnahmen (z.B. Gurt-/Helmpflicht, Methadonprogramm, „Fixerstuben“ etc.) geschaffen werden, die zu einem deutlichen Rückgang der Unfalltoten bzw. der Schwere von Verletzungen und Erkrankungen geführt haben. Weiterhin können rechtsmedizinische Untersuchungen unbekannte Gefahrenquellen aufdecken (z.B. Arbeitssicherheit, Wiederholungstat bei unerkannten Tötungsdelikten etc.), die von wesentlicher Bedeutung für die Sicherheit und Gesundheit der Gesellschaft sind (Thomsen und Schewe 1994).

\subsection{Gegenwärtige Situation}

In den letzten Jahren gibt es einen erheblichen Rückgang klinischer Obduktionen zu verzeichnen. In der Regel werden diese ausschließlich von Pathologen durchgeführt, sofern ein ärztliches bzw. wissenschaftliches Interesse an der Todesursache bzw. dem Grundleiden vorliegt (Madea 2006a). Voraussetzung für die Durchführung ist eine natürliche Todesart oder die Freigabe der Staatsanwaltschaft bei nicht natürlicher bzw. ungeklärter Todesart. So fiel der Anteil bundesweit von 10 \% (1980) auf 3,1 \% (1999), während der Anteil der rechtsmedizinisch ausgeführten Obduktionen nahezu konstant auf dem niedrigen Niveau von $2 \%$ stagnierte (Brinkmann et al. 2002). Für das Jahr 1999 konnte insgesamt eine Sektionsfrequenz von 5,3\% ermittelt werden. Trotz eines international anerkannten Indikationskataloges für eine Legalsektion nimmt Deutschland im internationalen Vergleich eine Schlussposition ein. Bundesweit werden etwa $8 \%$ aller Todesfälle als nicht natürlich bzw. unklar deklariert. Von diesen werden nur ein Viertel rechtsmedizinisch obduziert, da in den restlichen drei Vierteln Staatsanwälte bzw. Richter keine Hinweise auf ein Fremdverschulden ermitteln können (Brinkmann et al. 2002). Dem derzeitigen Leichenschau- und Sektionssystem wird eine nicht unerhebliche Anzahl unerkannter nicht natürlicher Todesfälle einschließlich Tötungsdelikten unterstellt (Brinkmann et al. 1997). Gleichzeitig wurden zahlreiche rechtsmedizinische Institute geschlossen bzw. Kürzungen innerhalb dieser vorgenommen (Latsch und Meyer 2005). Ausgelöst durch zahlreiche medienwirksame Publikationen im letzten Jahrzehnt erfreuten sich diese Themen zunehmend auch des öffentlichen Interesses: „Verordnete Vertuschung“ (Gatterburg und Koischwitz 2000), „Mord an der Rechtsmedizin“ (Rückert 2003), „Sparen mit tödlichen Folgen“ (Rückert 2000) und 
"Tote klagen nicht" (Latsch und Meyer 2005) sind nur einige Beispiele für Abhandlungen in der Presse.

\subsection{Zielsetzung der vorliegenden Arbeit}

In der vorliegenden Arbeit werden Sektionsprotokolle innerhalb eines 40-jährigen Zeitraumes untersucht. Retrospektiv lassen sich Aussagen über epidemiologische und phänomenologische Veränderungen treffen, die mit Ergebnissen anderer Institute verglichen werden können. Der Schwerpunkt dieser Arbeit liegt in der Betrachtung der Ursachen und Umstände nicht natürlicher Todesfälle. Die Entwicklungen werden vor dem Hintergrund der aktuellen Situation im Leichenschauund Obduktionswesen diskutiert. 


\section{Material und Methoden}

\subsection{Material}

Die Grundlage der Datenauswertung waren Sektionsprotokolle der Jahre von 1969 bis 1978 und von 1998 bis 2007 aus der Abteilung für Rechtsmedizin Göttingen. Anhand der Sektionsprotokolle, denen unter anderem auch die weiterführenden Untersuchungen (z.B. Mikrobiologie, Histologie, Alkohologie, Toxikologie, DNA, Neuropathologie etc.) beigefügt waren, wurden die relevanten Informationen erfasst. $\mathrm{Da}$ jedem eingelieferten Fall eine laufende Sektionsnummer zugeordnet wurde, musste zunächst festgestellt werden, bei welchen Fällen es sich um eine tatsächliche Obduktion oder lediglich um eine Leichenschau bzw. einen Knochenfund handelte. Dies konnte mithilfe der Sektionsbücher bestimmt werden. Die Anzahl der durchgeführten Obduktionen konnte nicht exakt ermittelt werden, weil die Sektionsbücher (mit Angabe des Einlieferungsgrundes und des Datums) für die Jahre von 1970 bis 1978 zum Untersuchungszeitpunkt nicht auffindbar waren. Daher gab es laufende Nummern, die einer Obduktion nicht sicher zuzuordnen waren. Diese wurden als „unklare Leichenfälle“ bezeichnet. Die Zahl der unklaren Leichenfälle wurde für den Zeitraum von 1970 bis 1978 mit 325 beziffert und für den von 2004 bis 2006 mit 10. Ursächlich für die 10 unklaren Leichenfälle der Jahre von 2004 bis 2006 waren, trotz eines vorhandenen Sektionsbuches, nicht nachvollziehbare Angaben oder durchgestrichene Einträge mit Leervergabe der Sektionsnummer. Von insgesamt 5.688 dokumentierten Fällen der Jahre von 1969 bis 1978 und 1998 bis 2007 konnten 5.353 als Obduktionsfälle kategorisiert werden, von denen 5.190 in diese Studie eingegangen sind. In dem Zeitraum von 1969 bis 1978 wurden 2.501 Fälle erfasst und in den Jahren 1998 bis 2007 weitere 2.689 Fälle. Die Zahl der nicht auswertbaren Obduktionen im gesamten Untersuchungszeitraum lag bei 163 (3 \%). Weitere 335 Sektionsnummern waren unbekannter Herkunft, unauffindbar oder wurden „leer" vergeben. 


\subsection{Methoden}

Die Datenerhebung der Jahre von 1969 bis 1978 und von 1998 bis 2007 erfolgte mittels Durchsicht der Obduktionsprotokolle. Diese waren zum Zeitpunkt der Datenerhebung ausschließlich in Form handschriftlicher und maschinengeschriebener Akten verfügbar. Um die studienrelevanten Daten in digitaler Form zu erfassen, wurden diese in eine Datenbank übertragen. Hierbei wurde mit dem Programm Microsoft ${ }^{\circledR}$ Access 2007 eine Eingabemaske erstellt, in die die notwendigen Informationen eingetragen wurden. Das Formular gliederte sich in fünf Teile. Zunächst wurden Basisdaten, Todesumstände sowie Besonderheiten des Falles eingetragen:

\footnotetext{
Sektionsnummer

Sektionsjahr

Sektionsmonat

Sektionsart

Sektionsort

Sterbe- oder Auffindeort

Leichenidentität

Geburtsjahr

Alter

Geschlecht

Leichenfundortbesichtigung

Intervall Tod/Auffindung bis zur Obduktion

postmortale Gesamtliegezeit

Leichenzustand

Todesart vor Obduktion

Todesart nach Obduktion

Todesart - Kategorie nach Obduktion

Besonderheiten

Behandlungsfehler
} 
- $\quad$ Evidenzgrad der Todesursache

- $\quad$ Sterbenstyp bei natürlichem Tod

- $\quad$ Basis der Todesursachendiagnostik

- Pflegezustand

Im zweiten Teil des Formulars wurden Angaben in Zusammenhang mit natürlichen Todesursachen eingetragen, die dem jeweiligen Organsystem zugeordnet und weiter spezifiziert wurden (s. Datenerhebungsbogen):

- $\quad$ Respirationstrakt

- $\quad$ Herz-Kreislauf-System

- $\quad$ zentrales Nervensystem

- $\quad$ Gastrointestinaltrakt

- Urogenitaltrakt

- $\quad$ endokrine Erkrankung

- $\quad$ tödliche Embolie

- Verbluten

- Infektionsgeschehen

- $\quad$ Stoffwechselentgleisung

- Tumor

- $\quad$ Fruchttod

- $\quad$ SIDS (Sudden Infant Death Syndrome)

Darauf folgte die Kategorie der nicht natürlichen Todesursachen mit den dazugehörigen Todesumständen, Formen der Gewalteinwirkung, Tatwerkzeugen und anatomischen Lokalisationen tödlicher Verletzungen: 
Form der Gewalteinwirkung:

- $\quad$ stumpfe Gewalt

o Tatwerkzeug bei Schlag

- $\quad$ Sturz

- $\quad$ scharfe Gewalt

- $\quad$ halbscharfe Gewalt

- $\quad$ Steckschuss/Durchschuss

o Waffenart

o Munition

o Entfernung des Schusses in Metern

- $\quad$ Strangulation

o Strangulationswerkzeug

- $\quad$ sonstiges Ersticken

- Bolustod

- $\quad$ Ertrinken

- $\quad$ thermische Schädigung

- $\quad$ Unterkühlung

- Stromtod/Blitzschlag

- Verhungern/Verdursten

- Intoxikation

Anatomische Lokalisation:

- $\quad$ verletzte Körperregion

Weitere Angaben zur Todesursache:

- $\quad$ systemische bzw. direkte Todesursachen

- $\quad$ mittelbare Todesursachen 
Todesumstand:

- $\quad$ Verkehrsunfall

- $\quad$ Neugeborene/Föten

- $\quad$ Tod nach medizinischen Maßnahmen

Im vierten Teil wurden qualitative und quantitative Angaben zu toxikologischen Untersuchungen dokumentiert. Schließlich wurden in dem fünften und somit letzten Teil zusätzliche Notizen, ergänzende Informationen oder Daten eingegeben, die den o.g. Kategorien nicht zuzuordnen waren (z.B. Sonstiges).

Die Daten wurden nach der Eingabe in Microsoft ${ }^{\circledR}$ Access in das Programm Statistica ${ }^{\circledR}$ übertragen, mit dem weitere Auswertungen durchgeführt wurden.

Die Ergebnisse wurden als absolute und prozentuale Werte vergleichsweise gegenübergestellt. Die Basis der prozentualen Werte bilden die absoluten Werte innerhalb der Gruppierung eines Untersuchungszeitraumes, bezogen auf die Gesamtzahl der Fälle des Untersuchungszeitraumes. Zur Ermittlung der Veränderung dieser relativen Anteile zwischen den Untersuchungszeiträumen wurde die relative Änderung $(\Delta)$ der prozentualen Anteile ermittelt.

Studienrelevante Informationen zur Todesursache und zu den Todesumständen wurden der Vorgeschichte bzw. dem vorläufigen/endgültigen Gutachten entnommen. Es erfolgte keine Neubewertung bzw. Neuinterpretation von Befunden/Diagnosen. Da zum Untersuchungszeitpunkt staatsanwaltliche Ermittlungsakten bzw. Gerichtsurteile nicht immer vorlagen, konnte die juristische Zuordnung des Falles nicht berücksichtigt werden (z. B. Behandlungsfehler, Kindstötung, Vernachlässigung oder Tötungsdelikte). In einigen Fällen wurde jedoch auf Ermittlungsergebnisse bzw. Urteile verwiesen, die entsprechend mit erfasst wurden.

Der Datenerhebungsbogen (mit Eingabemöglichkeiten) ist dem Anhang zu entnehmen. 


\section{Ergebnisse}

\subsection{Basisdaten}

\subsubsection{Sektionszahlen}

Die Verteilung der Sektionszahlen ist in Tabelle 1 wiedergegeben.

Mit 2.501 (48,2 \%) Sektionen im ersten sowie 2.689 (51,8 \%) im zweiten Zeitraum verteilten sich die Fälle nahezu gleichmäßig auf beide Zeiträume. Innerhalb des gesamten Betrachtungszeitraumes wurden durchschnittlich 432,5 Sektionen monatlich durchgeführt. Die höchste Obduktionsquote ergab sich für den Monat Dezember $(n=476,9,2 \%)$ und die niedrigste für den Monat September $(n=387$, $7,5 \%)$.

\begin{tabular}{|l|c|c|c|c|c|c|c|}
\cline { 2 - 8 } \multicolumn{1}{c|}{} & Gesamt & & $\mathbf{1 9 6 9 - 1 9 7 8}$ & & $\mathbf{1 9 9 8 - 2 0 0 7}$ & & rel. Änderung \\
\cline { 2 - 8 } \multicolumn{1}{c|}{} & Anzahl & in \% & Anzahl & in \% & Anzahl & in \% & in \% \\
\hline Januar & 447 & 8,6 & 198 & 7,9 & 249 & 9,2 & $\mathbf{+ 1 7}$ \\
\hline Februar & 452 & 8,7 & 221 & 8,9 & 231 & 8,6 & $\mathbf{- 2 , 8}$ \\
\hline März & 440 & 8,5 & 207 & 8,3 & 233 & 8,7 & $\mathbf{+ 4 , 7}$ \\
\hline April & 434 & 8,4 & 211 & 8,4 & 223 & 8,3 & $\mathbf{- 1 , 7}$ \\
\hline Mai & 451 & 8,7 & 206 & 8,2 & 245 & 9,1 & $\mathbf{+ 1 0 , 6}$ \\
\hline Juni & 422 & 8,1 & 207 & 8,3 & 215 & 8 & $\mathbf{- 3 , 4}$ \\
\hline Juli & 411 & 7,9 & 210 & 8,4 & 201 & 7,5 & $\mathbf{- 1 1}$ \\
\hline August & 425 & 8,2 & 183 & 7,3 & 242 & 9 & $\mathbf{+ 2 3}$ \\
\hline September & 387 & 7,5 & 176 & 7 & 211 & 7,8 & $\mathbf{+ 1 1 , 5}$ \\
\hline Oktober & 418 & 8 & 200 & 8 & 218 & 8,1 & $\mathbf{+ 1 , 4}$ \\
\hline November & 427 & 8,2 & 232 & 9,3 & 195 & 7,3 & $\mathbf{- 2 1 , 8}$ \\
\hline Dezember & 476 & 9,2 & 250 & 10 & 226 & 8,4 & $\mathbf{- 1 5 , 9}$ \\
\hline Gesamt & $\mathbf{5 . 1 9 0}$ & 100 & $\mathbf{2 . 5 0 1}$ & 100 & $\mathbf{2 . 6 8 9}$ & 100 & \\
\hline
\end{tabular}

Tab. 1: Sektionszahlen 1969-1978 und 1998-2007 


\subsubsection{Geschlechterverteilung}

Die Geschlechterverteilung ist in Abbildung 1 dargestellt.

Für den gesamten Untersuchungszeitraum zeigte sich eine Verteilung der Sektionsfälle von 65,6 \% (n=3.404) für das männliche sowie von 34,4\% $(n=1.786)$ für das weibliche Geschlecht. Die relative Änderung des Anteils männlicher Sterbefälle betrug $+1,5 \%$, die der weiblichen $-2,7 \%$.

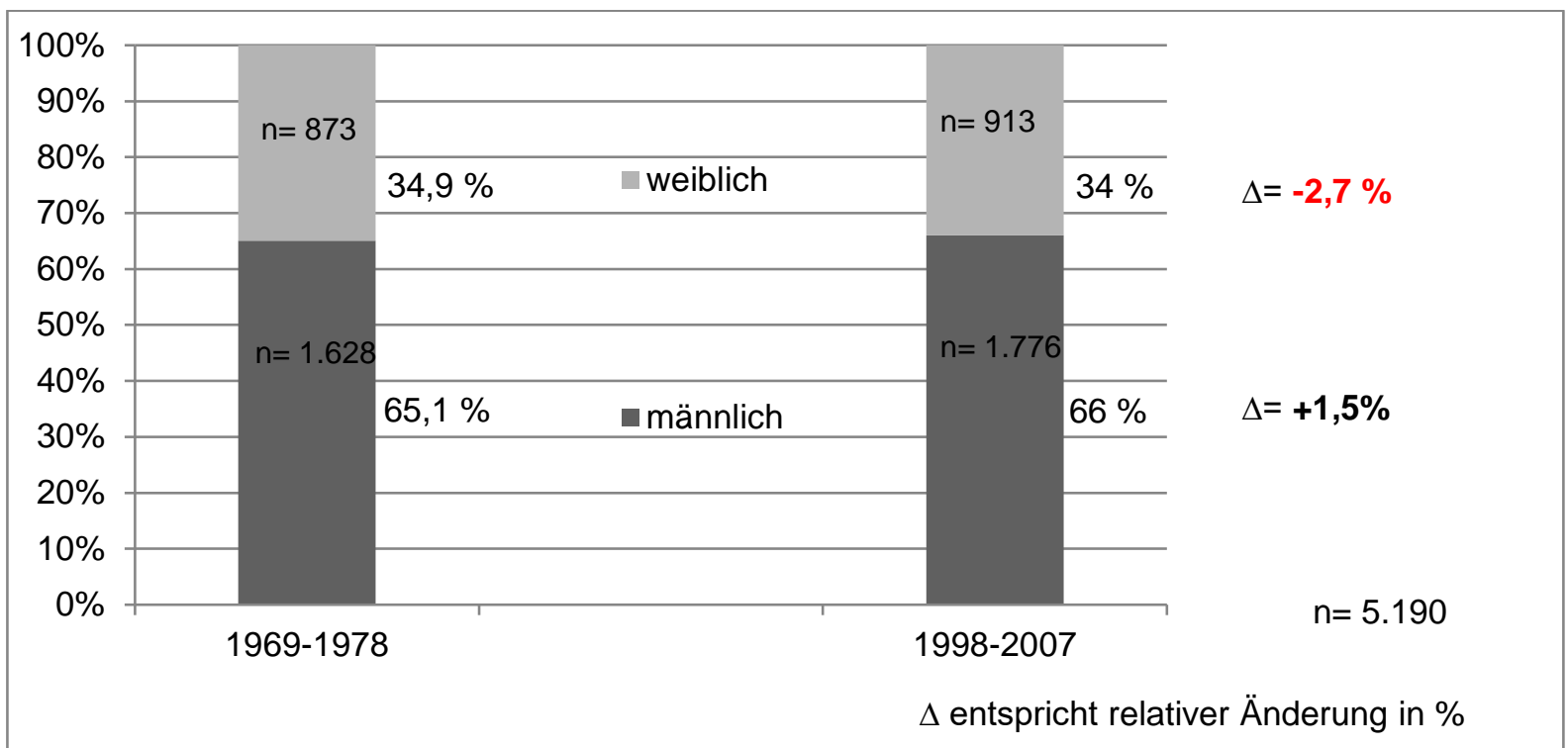

Abb. 1: Geschlechterverteilung 1969-1978 und 1998-2007 


\subsubsection{Sektionsort}

Die Verteilung der Sektionsorte ist Abbildung 2 zu entnehmen.

Die meisten Obduktionen wurden in Form von Außensektionen durchgeführt. Es ergaben sich jedoch große Veränderungen innerhalb der Gruppen. Außensektionen in Hessen sind im zweiten Betrachtungszeitraum neu hinzugekommen ( $n=1.046)$ und waren im ersten Zeitraum praktisch nicht vorhanden $(n=1)$. Desweiteren hat der Anteil der Sektionen im hiesigen Institut zugenommen $(\Delta=+16,1 \%)$. Stark rückläufig war der Anteil der Außensektionen in Niedersachsen $(\Delta=-53,5 \%)$. Die übrigen Sektionsorte fielen durch ihre geringe Zahl kaum ins Gewicht.

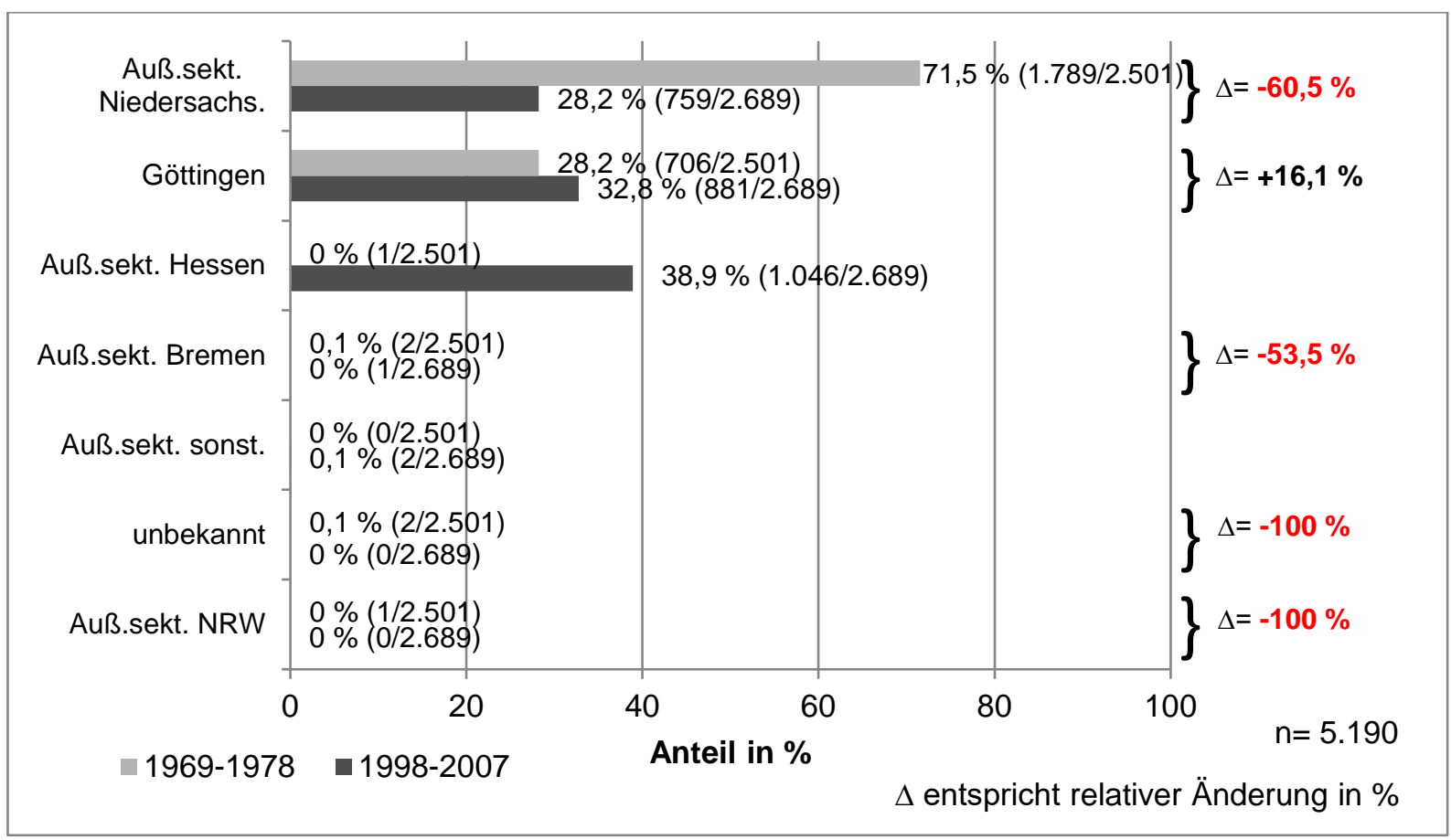

Abb. 2: Sektionsorte 1969-1978 und 1998-2007 


\subsubsection{Sektionsart}

Die Verteilung der Sektionsart ist in Abbildung 3 wiedergegeben.

Die gerichtliche Sektion machte in beiden Zeiträumen den Großteil aller Sektionen aus $(95,1 \%, n=4.938)$. Die relative Zunahme innerhalb dieser Gruppe betrug 5,3 \%. Ebenfalls zugenommen $(\Delta=+293,5 \%)$ hat der Anteil der sonstigen Sektionsarten (Berufsgenossenschaft, Versicherung, privat etc.). Die relative Abnahme der klinischen bzw. Verwaltungssektion betrug 93,5 \%.

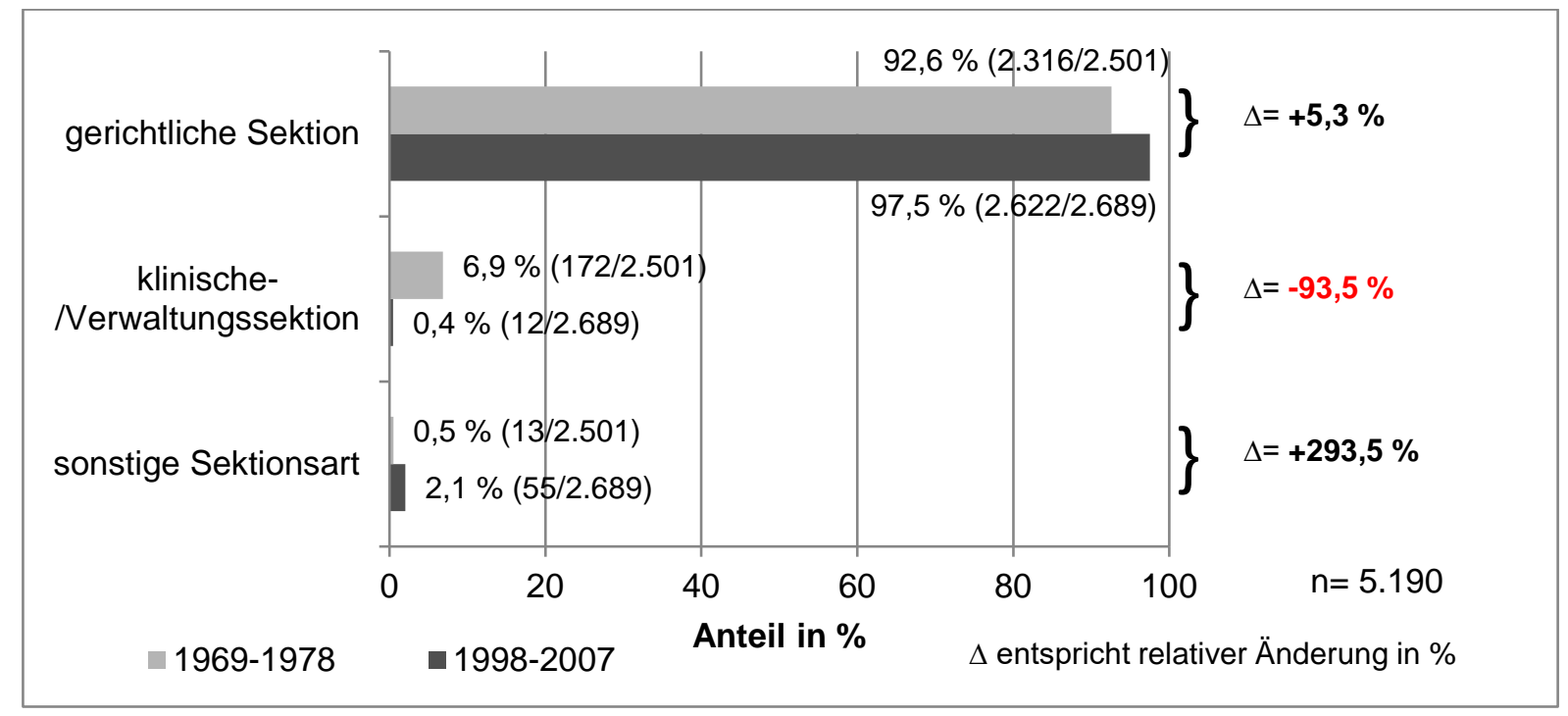

Abb. 3: Sektionsart 1969-1978 und 1998-2007 


\subsubsection{Sterbe-/Auffindeort}

Die Häufigkeit der Sterbe- bzw. Auffindeorte der Verstorbenen können der folgenden Grafik (Abb. 4) entnommen werden.

Die meisten Funde gab es im häuslichen Umfeld ( $n=2.213,42,6 \%, \Delta=+15,5 \%)$. An zweiter Stelle folgten mit 1.259 Fällen die Krankenhäuser, Psychiatrien und Pflegeheime (24,3\%, $\Delta=-21,3 \%)$. Mit 773 Fällen $(7,4 \%, \Delta=-23,8 \%)$ standen die Funde in der Öffentlichkeit an dritter Stelle. Es folgten die Funde im Straßenverkehr mit 385 Fällen $(7,4 \%, \Delta=-18,3 \%)$ sowie am Arbeitsplatz mit 114 Fällen (2,2\%, $\Delta=-65,3 \%)$. Deutlich zugenommen haben Leichenfunde im Polizeigewahrsam und in Justizvollzugsanstalten $(n=69, \Delta=+265,4 \%)$.

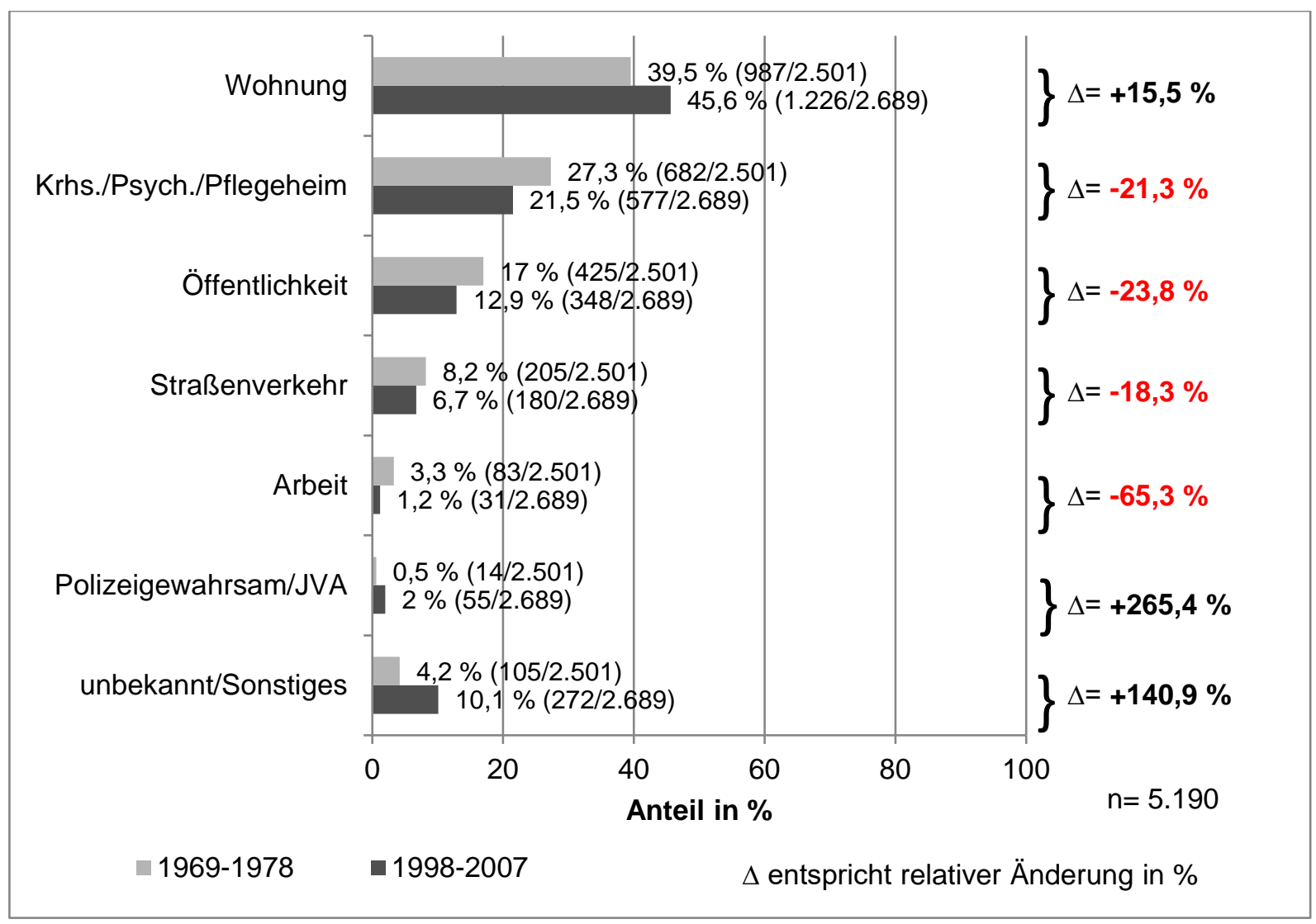

Abb. 4: Sterbe-/Auffindeort 1969-1978 und 1998-2007 


\subsubsection{Leichenidentität}

Die Verteilung der Leichenidentität ist Tabelle 2 zu entnehmen.

Demnach konnte in $97,5 \%$ aller Fälle $(n=5.058)$ die Identität zum Obduktionszeitpunkt bestimmt werden. Der Anteil dieser Gruppe ist um 0,3 \% (relative Änderung) angestiegen. In 92 Fällen $(1,8 \%, \Delta=+32,2 \%)$ war die Identität zum Zeitpunkt der Obduktion zunächst unbekannt, konnte aber im Verlauf ermittelt werden. Der Anteil unbekannter Identitäten fiel von 1,2\% $(n=30)$ auf $0,4 \%(n=10)$.

\begin{tabular}{|l|c|c|c|c|c|c|c|}
\hline & Gesamt & & $\mathbf{1 9 6 9 - 1 9 7 8}$ & & $\mathbf{1 9 9 8 - 2 0 0 7}$ & & rel. Änderung \\
\hline & Anzahl & in \% & Anzahl & in \% & Anzahl & in \% & in \% \\
\hline bekannt & 5.058 & 97,5 & 2.433 & 97,3 & 2.625 & 97,6 & $\mathbf{+ 0 , 3}$ \\
\hline $\begin{array}{l}\text { primär unbekannt } \\
\text { später identifiziert }\end{array}$ & 92 & 1,8 & 38 & 1,5 & 54 & 2 & $\mathbf{+ 3 2 , 2}$ \\
\hline unbekannt & 40 & 0,7 & 30 & 1,2 & 10 & 0,4 & $\mathbf{- 6 9}$ \\
\hline Gesamt & $\mathbf{5 . 1 9 0}$ & 100 & $\mathbf{2 . 5 0 1}$ & 100 & $\mathbf{2 . 6 8 9}$ & 100 & \\
\hline
\end{tabular}

Tab. 2: Leichenidentität 1969-1978 und 1998-2007 


\subsubsection{Sterbealter}

Die Verteilung des Lebensalters zum Todeszeitpunkt ist in Abbildung $5 \mathrm{zu}$ dargestellt.

In 34 Fällen $(0,7 \%, \Delta=-71,4 \%)$ fehlte eine Angabe zum Lebensalter, bzw. konnte das Alter trotz Zusatzuntersuchungen nicht ermittelt werden. In weiteren 14 Fällen $(0,3 \%)$ handelte es sich um eine Totgeburt. In insgesamt 816 Fällen (15,7 \%) konnte das Alter zwischen dem begonnenen ersten Lebensjahr und dem vollendeten 18. Lebensjahr eingeordnet werden. Innerhalb dieser Gruppe gab es einen relativen Rückgang von 53,2 \%. Die Gruppe der 19- bis 40-Jährigen war mit einem Anteil von $31,2 \%(n=1.619)$ vertreten, entsprechend einem relativen Rückgang von $14 \%$. Angestiegen ist der Anteil der Altersgruppe 41. - 65. Lebensjahr ( $\Delta=+31,4 \%)$. Das mittlere Sterbealter lag im ersten Betrachtungszeitraum bei 37,8 Jahren und der gruppierte Median bei 37 Jahren. Im zweiten Betrachtungszeitraum (1998-2007) lag der Mittelwert bei 46,8 Jahren und der gruppierte Median bei 46. Somit war das Sterbealter um 9 Jahre angestiegen.

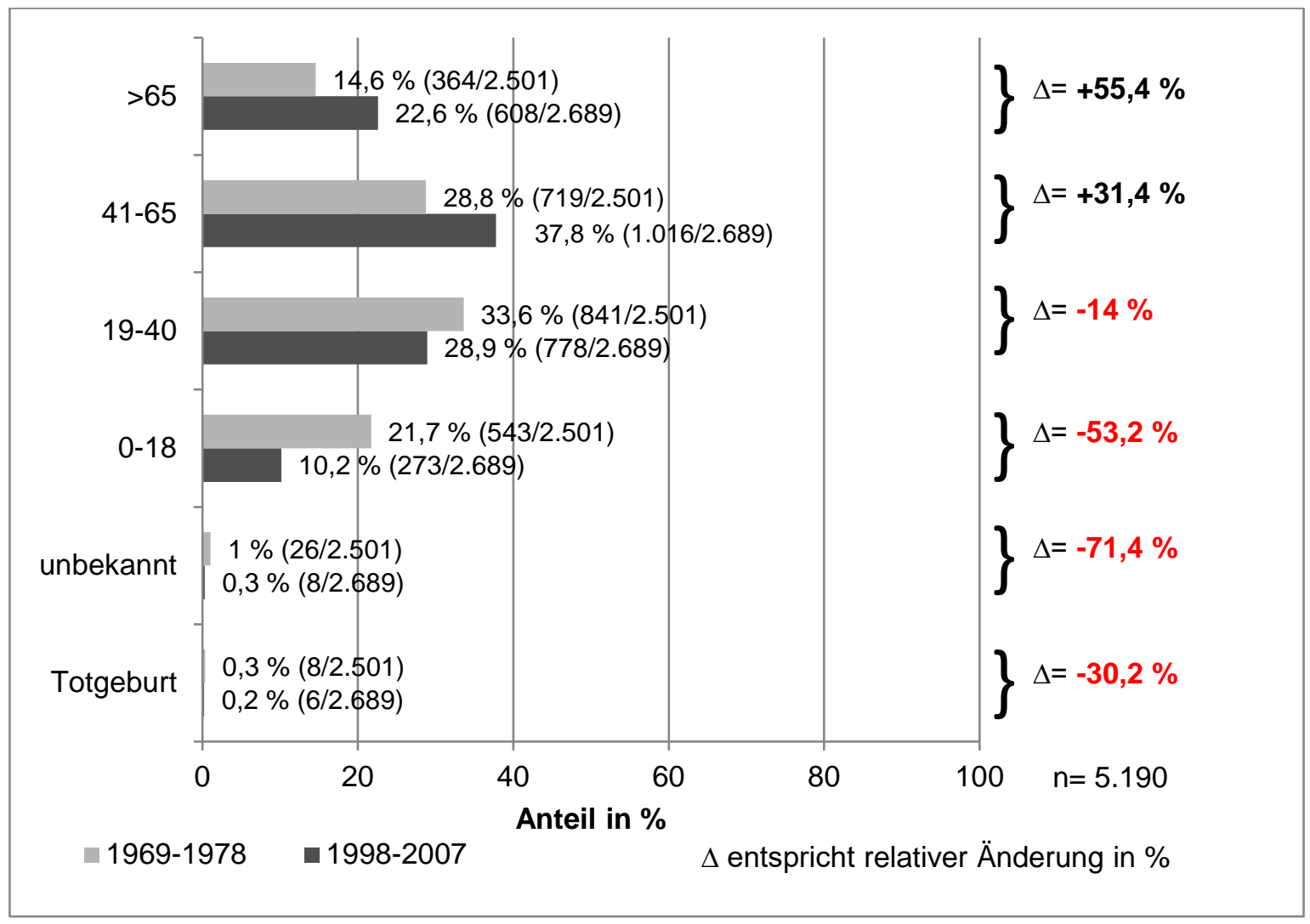

Abb. 5: Sterbealter in Jahren 1969-1978 und 1998-2007 


\subsubsection{Intervall vom Auffindezeitpunkt bis zur Obduktion}

Die Anzahl der Tage, die zwischen dem Zeitpunkt des Leichenfundes und der Obduktion jeweils verstrichen waren, ist Tabelle 3 zu entnehmen.

Demnach betrug die durchschnittliche Dauer im ersten Betrachtungszeitraum (19691978) 2,9 Tage und in den Jahren von 1998 bis 2007 5,8 Tage. Es wurde auch der Median, der in beiden Betrachtungszeiträumen 1 Tag war, ermittelt.

\begin{tabular}{|l|c|c|}
\cline { 2 - 3 } \multicolumn{1}{c|}{} & $1969-1978$ & $1998-2007$ \\
\cline { 2 - 3 } \multicolumn{1}{c|}{} & Tage & Tage \\
\hline Durchschnitt & 2,9 & 5,8 \\
\hline Median & 1 & 1 \\
\hline
\end{tabular}

Tab. 3: Intervall Auffindezeitpunkt bis zur Obduktion 1969-1978 und 1998-2007

\subsubsection{Postmortale Gesamtliegezeit}

Die Zahl der Tage (teilweise geschätzt) zwischen dem Todeszeitpunkt und der Obduktion zeigt Tabelle 4.

Die durchschnittliche postmortale Liegezeit konnte mit 5,4 Tagen (1969-1978) sowie 8,2 Tagen (1998-2007) beziffert werden. Der Median lag in dem früheren Zeitraum bei 2 Tagen, im späteren Zeitraum betrug dieser 1 Tag.

\begin{tabular}{|l|c|c|}
\cline { 2 - 3 } \multicolumn{1}{c|}{} & $1969-1978$ & $1998-2007$ \\
\cline { 2 - 3 } \multicolumn{1}{c|}{} & Tage & Tage \\
\hline Durchschnitt & 5,3 & 8,2 \\
\hline Median & 2 & 1 \\
\hline
\end{tabular}

Tab. 4: Postmortale Gesamtliegezeit 1969-1978 und 1998-2007 


\subsubsection{Leichenzustand}

In Abbildung 6 wird dargestellt, in welchem Zustand sich wie viele Leichen zum jeweiligen Obduktionszeitpunkt befanden.

Demnach wurde bei 4.086 Leichen ( $\Delta=+9,1 \%$ ) der Zustand "frisch/keine Fäulnis“ erhoben. Am zweithäufigsten handelte es sich in 479 Fällen $(\Delta=-46,9 \%)$ um Leichen mit Anzeichen einer leichten Fäulnis. Der Zustand einer mittelgradigen Fäulnis lag in 241 Fällen $(\Delta=+10,8 \%)$ vor. In 253 Fällen $(\Delta=+54,7 \%)$ wurde der Zustand als „starke Fäulniszeichen/Madenbefall“ klassifiziert. Teilskelettierte bzw. skelettierte Leichen lagen in 72 Fällen $(\Delta=-7 \%)$ vor. Letztlich war in 59 Fällen $(\Delta=-85,4 \%)$ der Befund unklar bzw. waren Informationen nicht eruierbar.

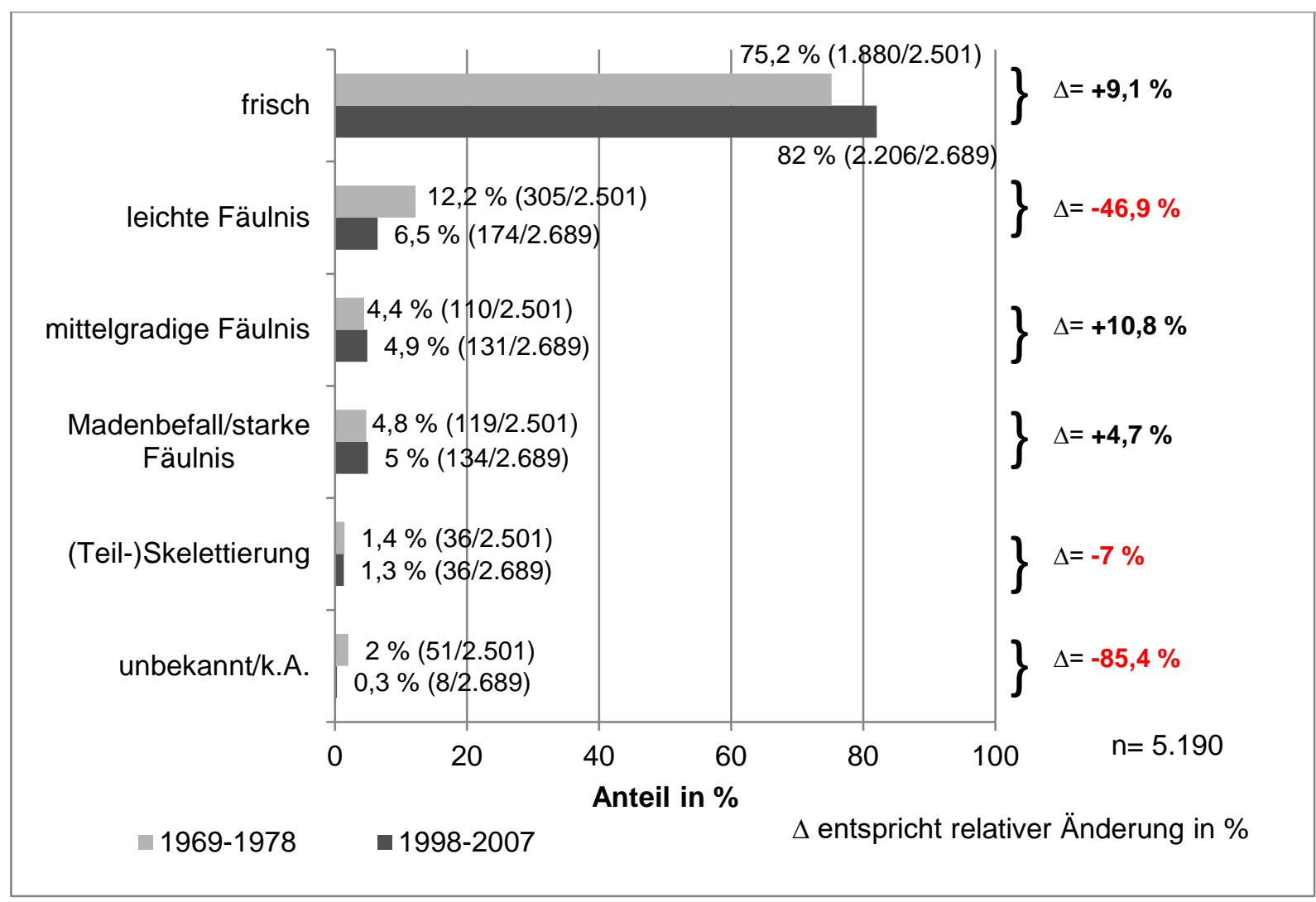

Abb. 6: Leichenzustand 1969-1978 und 1998-2007 


\subsubsection{Todesart nach Obduktion}

Abbildung 7 stellt den Anteil der Todesarten nach Obduktion dar.

Demnach wurden 59,4 \% ( $n=3.075, \Delta=-16,1 \%)$ aller Sektionsfälle einer nicht natürlichen Todesart zugeordnet. Der Anteil natürlicher Todesfälle lag bei 33,4\% ( $\mathrm{n}=1.731, \Delta=+29,8 \%)$. In 371 Fällen $(7,2 \%, \Delta=+29,4 \%)$ wurde die Todesart mit „ungeklärt“ angegeben. Totgeburten wurden in 13 Fällen dokumentiert (in Abb. 7 nicht abgebildet).

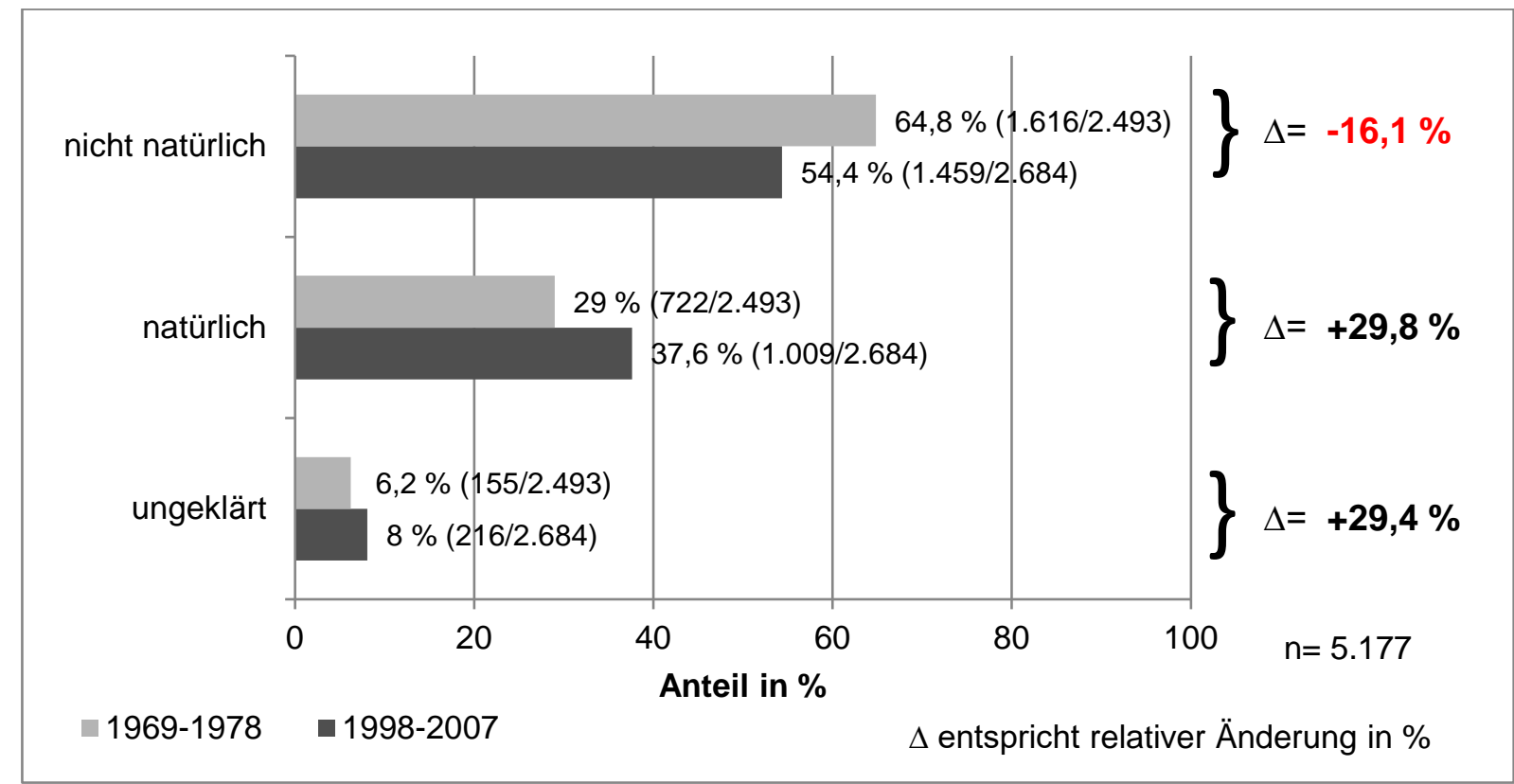

Abb. 7: Todesart nach Obduktion 1969-1978 und 1998-2007 


\subsubsection{Todesart - Kategorie nach Obduktion}

Die Kategorie der Todesart enthielt nähere Angaben zu den Todesumständen. Die Verteilung ist der Abbildung 8 zu entnehmen.

In 4.162 Fällen (80,2 \%) konnte eine Eintragung zur Todesart (Kategorie) vorgenommen werden. Angestiegen sind tödliche Intoxikationen und Suizide $(\Delta=+52,6 \%$ und $\Delta=+35,1 \%)$. Der Anteil männlicher Suizidenten im gesamten Untersuchungszeitraum betrug 73,2 \% (in Abb. 8 nicht abgebildet). Die relative Abnahme der sonstigen Unfälle (z.B. häuslicher Unfall, Sport- und Freizeitunfall etc.) betrug 30,4 \%. Ebenfalls rückläufig war der Anteil der Tötungsdelikte $(\Delta=-58,1 \%)$.

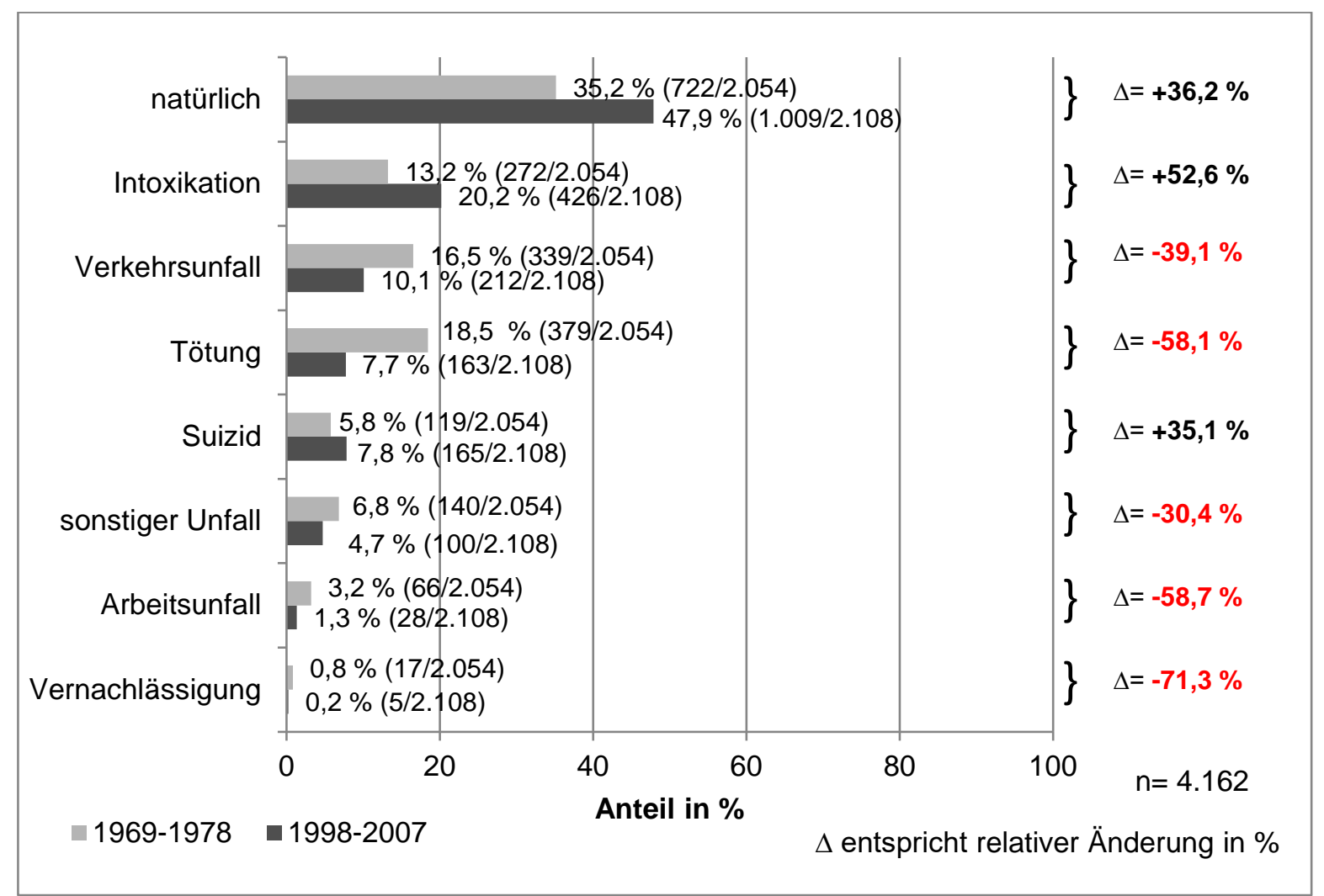

Abb. 8: Todesart - Kategorie nach Obduktion 1969-1978 und 1998-2007 


\subsubsection{Besonderheiten}

Die Verteilung der Besonderheiten zeigt Tabelle 5.

Ein starker Rückgang konnte bei Todesfällen festgestellt werden, die in Verbindung mit einem Schwangerschaftsabbruch standen ( $\mathrm{n}=34, \Delta=-74,6 \%$,). Ebenfalls gesunken war der Anteil von Fällen, in denen ein Sexualdelikt ( $n=28, \Delta=-96,4 \%)$ und eine Schwangerschaft $(n=22, \Delta=-84,5 \%)$ dokumentiert wurden. Des Weiteren nahmen Wasserleichenfunde $(n=220, \Delta=-24,1 \%)$ sowie Verdachtsfälle des SIDS (Sudden Infant Death Syndrom) ab ( $\mathrm{n}=293, \Delta=-53,7 \%)$. Stark angestiegen sind hingegen Todesfälle in Verbindung mit Drogenkonsum ( $\mathrm{n}=259, \Delta=+1.615,4 \%)$.

\begin{tabular}{|l|c|c|c|c|c|c|c|}
\cline { 2 - 9 } \multicolumn{1}{l|}{} & Gesamt & & $\mathbf{1 9 6 9 - 1 9 7 8}$ & & $\mathbf{1 9 9 8 - 2 0 0 7}$ & & rel. Änderung \\
\cline { 2 - 9 } \multicolumn{1}{l|}{ Anzahl } & in \% & Anzahl & in \% & Anzahl & in \% & in \% \\
\hline V.a. auf SIDS & 293 & 26,5 & 199 & 36,5 & 94 & 16,8 & $-\mathbf{5 3 , 7}$ \\
\hline "Drogentoter" & 259 & 23,5 & 14 & 2,6 & 245 & 44 & $\mathbf{+ 1 . 6 1 5 , 4}$ \\
\hline Brandleiche & 220 & 19,9 & 124 & 22,7 & 96 & 17,2 & $-\mathbf{2 4 , 1}$ \\
\hline Mehrleichenfund & 99 & 9 & 46 & 8,4 & 53 & 9,5 & $\mathbf{+ 1 2 , 9}$ \\
\hline Schwangerschaftsabbruch & 60 & 5,4 & 34 & 6,2 & 26 & 4,6 & $-\mathbf{2 5}$ \\
\hline Sexualdelikt & 34 & 3,1 & 27 & 4,9 & 7 & 1,3 & $-\mathbf{7 4 , 6}$ \\
\hline Exhumierung & 28 & 2,5 & 27 & 4,9 & 1 & 0,2 & $\mathbf{- 9 6 , 4}$ \\
\hline V.a. erweiterten Suizid & 23 & 2,1 & 16 & 2,9 & 7 & 1,3 & $\mathbf{- 5 7 , 1}$ \\
\hline Schwangerschaft & 22 & 2 & 19 & 3,5 & 3 & 0,5 & $\mathbf{- 8 4 , 5}$ \\
\hline Nachsektion & 15 & 1,4 & 9 & 1,6 & 6 & 1,1 & $\mathbf{- 3 4 , 6}$ \\
\hline Kindesmisshandlung & 15 & 1,4 & 8 & 1,5 & 7 & 1,3 & $\mathbf{- 1 4 , 2}$ \\
\hline autoerotischer Unfall & 7 & 0,6 & 2 & 0,4 & 5 & 0,9 & $\mathbf{+ 1 4 5 , 1}$ \\
\hline Mors in actu & 3 & 0,3 & 1 & 0,2 & 2 & 0,4 & $\mathbf{+ 9 6 , 1}$ \\
\hline Gesamt & $\mathbf{1 . 1 0 3}$ & 100 & $\mathbf{5 4 6}$ & 100 & $\mathbf{5 5 7}$ & 100 & \\
\hline
\end{tabular}

Tab. 5: Besonderheiten 1969-1978 und 1998-2007 


\subsubsection{Behandlungsfehler}

Die Verteilung der Sektionszahlen, die aufgrund eines Behandlungsfehlervorwurfs durchgeführt wurden, ist in Abbildung 9 dargestellt.

Im Zeitraum von 1969-1978 wurden lediglich 56 Fälle mit Verdacht auf Behandlungsfehler untersucht. Diese Zahl stieg auf 263 an (1998-2007). Der Anteil bestätigter Fälle fiel deutlich ( $\Delta=-84 \%)$, ebenso wie der der ungeklärten Fälle $(\Delta=-61,4 \%)$. Hingegen ist der Anteil der Fälle, in denen ein Behandlungsfehler ausgeschlossen wurde, um 71,1 \% (relative Änderung) gestiegen. Das mittlere Sterbealter innerhalb dieser Gruppe stieg von 39,7 (1969-1978) auf 60,7 (1998-2007) Jahre an (in Abb. 9 nicht abgebildet).

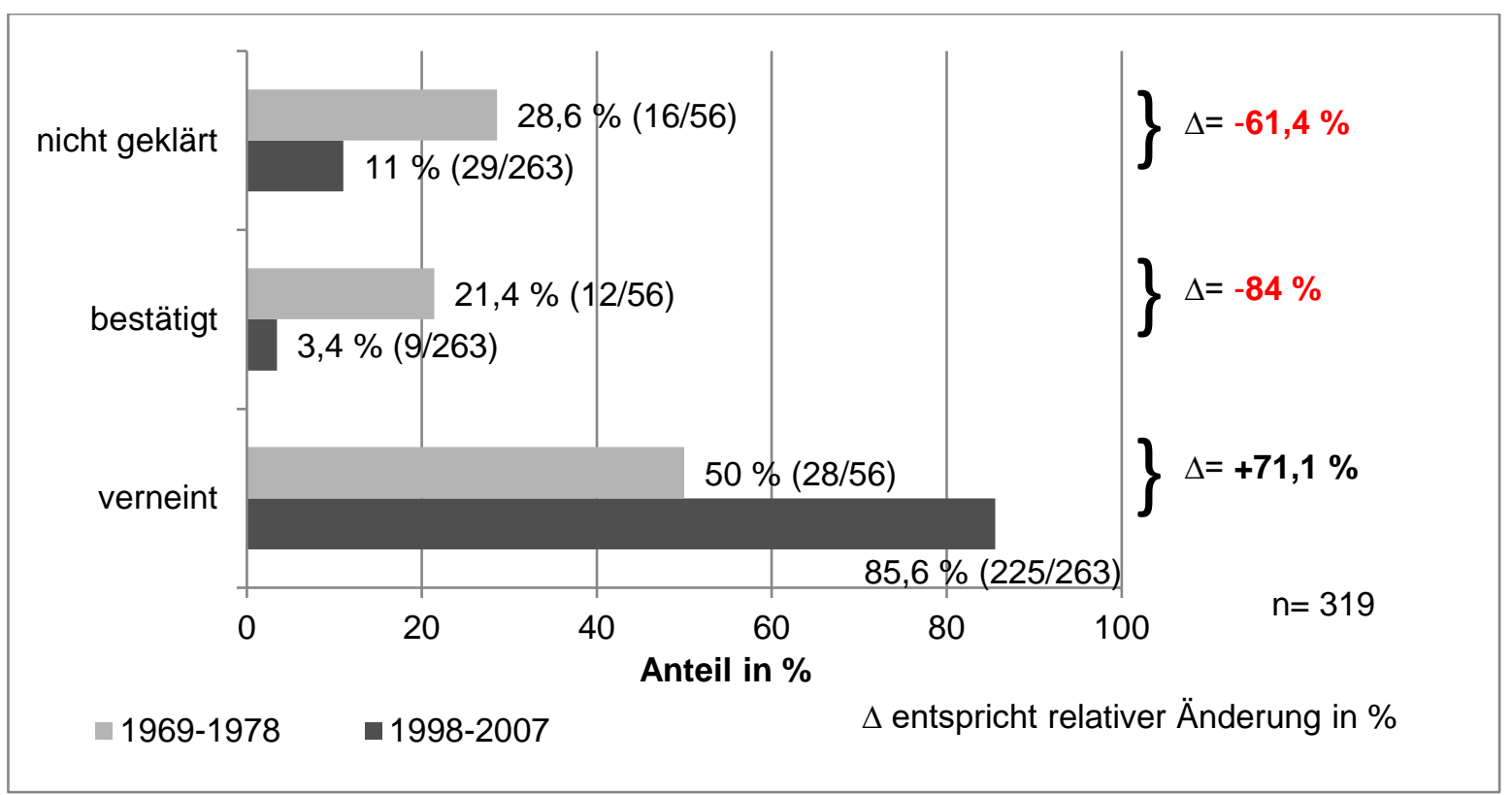

Abb. 9: Behandlungsfehler 1969-1978 und 1998-2007 


\subsubsection{Evidenzgrad der Todesursache}

Der Evidenzgrad der Todesursache lässt sich in der Literatur in drei Gruppen unterteilen (Madea 2007, S. 25):

1. Befunde, die aufgrund ihres Schweregrades und ihrer Lokalisation für sich allein und ohne Einschränkung den Tod eines Menschen erklären, wie z.B. ein rupturiertes Hirnbasisarterienaneurysma mit tödlicher Subarachnoidalblutung oder der rupturierte Myokardinfarkt.

2. Organveränderungen, die den Tod erklären, aber nicht die Akuität des Todeseintritts. Ein Beispiel wäre die akute Koronarinsuffizienz. Das morphologische Substrat, die schwere Arteriosklerose, bestand zweifellos auch bereits am Tag zuvor, eine äußere Belastung wie körperliche Arbeit bei schwülem Wetter ist jedoch das hinzutretende Ereignis für den Todeseintritt zum gegebenen Zeitpunkt.

3. Todesfälle, bei denen trotz sorgsamer Untersuchungen keine Todesursache zu finden ist.

Die Verteilung ist in Abbildung 10 wiedergegeben.

Mit einem Anteil von 65,2\% $(n=3.382, \Delta=+0,7 \%)$ liegen Befunde der Gruppe 1 an erster Stelle. Es folgen der Anteil der Gruppe 2 mit 25,3\% ( $n=1.311, \Delta=-19,1 \%)$ sowie der Gruppe 3 mit 6,1 \% ( $n=314, \Delta=+641,4 \%)$. Der o.g. Einteilung waren $3,5 \%$ der Fälle nicht sicher zuordenbar $(n=183, \Delta=-72,3 \%)$.

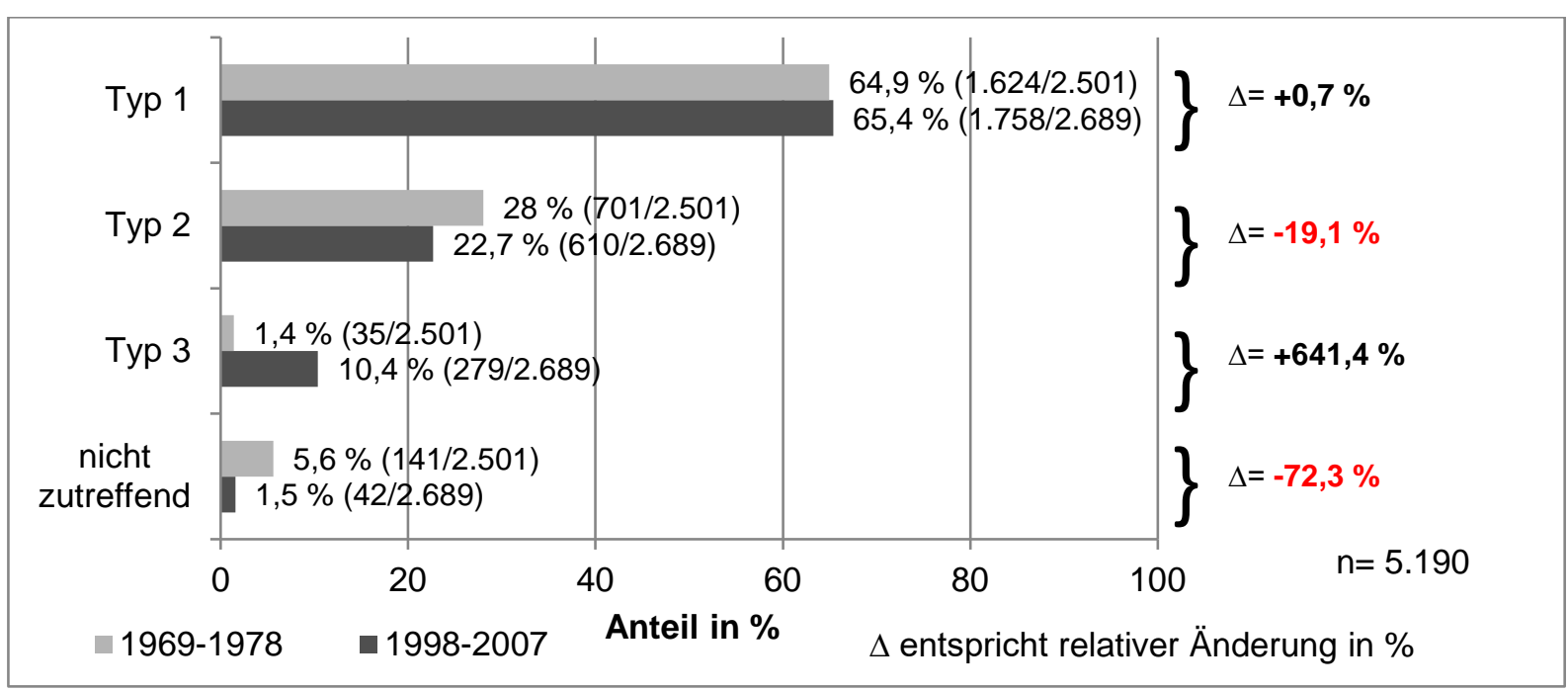

Abb. 10: Evidenzgrad der Todesursache 1969-1978 und 1998-2007 


\subsubsection{Sterbenstyp bei natürlichem Tod}

Die Sterbenstypen lassen sich folgendermaßen einteilen (Madea 2007, S. 25-26):

1. Linearer Sterbenstyp: Grundleiden und Todesursache liegen in einem Organsystem.

2. Divergierender Sterbenstyp: Organspezifisches Grundleiden, jedoch organunspezifische Todesursache.

3. Konvergierender Sterbenstyp: In verschiedenen Organsystemen gelegene Grundleiden führen über eine gemeinsame pathogenetische Endstrecke zum Tod.

4. Komplexer Sterbenstyp: In verschiedenen Organsystemen gelegene Grundleiden mit mehreren organspezifischen Todesursachen.

In dieser Einteilung steht der lineare Sterbenstyp mit 85,6\% $(n=1.470, \Delta=+1,3 \%)$ an der Spitze. Es folgen der divergierende Typ mit 8,3\% ( $n=143, \Delta=-7 \%)$ sowie der komplexe Typ mit 5,4\% ( $n=93, \Delta=+7,3 \%)$. An letzter Stelle steht der konvergierende Typ mit einem Anteil von 0,7\% ( $n=12, \Delta=-74,2 \%)$. Die Verteilung der Sterbenstypen verdeutlicht Abbildung 11.

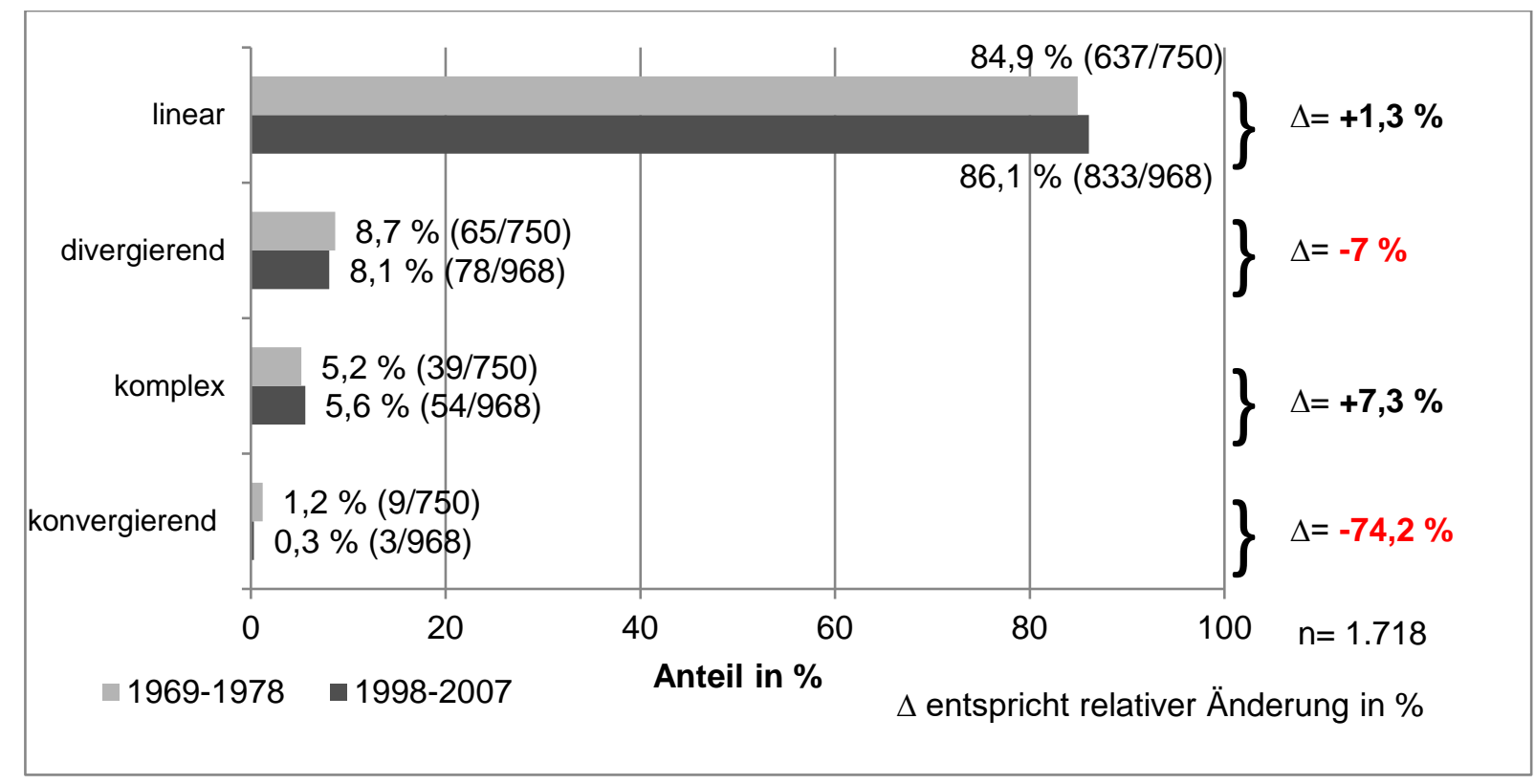

Abb. 11: Sterbenstypen bei natürlichem Tod 1969-1978 und 1998-2007 


\subsubsection{Basis der Todesursachendiagnostik}

Die zur Todesursachenklärung durchgeführten diagnostischen Untersuchungen wurden in Tabelle 6 zusammengefasst.

Der Anteil der Fälle, in denen die Obduktion ohne weiterführende Untersuchung durchgeführt wurde, stieg um 60 \% (relative Änderung). Ebenfalls angestiegen ist der Anteil der Gruppe Alkohologie/Toxikologie ( $\Delta=+53,2$ \%). Die Gruppe der „Sonstigen“ ist verhältnismäßig groß ausgefallen, da bestimmte Untersuchungen und Kombinationen aus dem früheren Zeitraum (u.a. ballistische Untersuchungen, Blutgruppenbestimmungen und Faseruntersuchungen) im späteren Zeitraum kaum noch durchgeführt wurden. Andererseits sind neue Untersuchungsmethoden (z.B. DNA-Analysen) hinzugekommen. Die relative Zunahme dieser Gruppe betrug 54 \%. Demgegenüber stehen Rückgänge bei zusätzlichen Alkoholuntersuchungen $(\Delta=-54,1 \%)$, histologischen Untersuchungen $(\Delta=-74,4 \%)$, den Kombinationen Alkohol/Histologie/Toxikologie $(\Delta=-68,1 \%)$, Alkohol/Histologie $(\Delta=-96,4 \%)$ sowie der Toxikologie $(\Delta=-0,8 \%)$.

\begin{tabular}{|l|c|c|c|c|c|c|c|}
\cline { 2 - 8 } \multicolumn{1}{c|}{} & Gesamt & & $\mathbf{1 9 6 9 - 1 9 7 8}$ & & $\mathbf{1 9 9 8 - 2 0 0 7}$ & & rel. Änderung \\
\cline { 2 - 9 } \multicolumn{1}{c|}{} & Anzahl & in \% & Anzahl & in \% & Anzahl & in \% & in \% \\
\hline Obduktion & 1.787 & 34,4 & 657 & 26,3 & 1.130 & 42 & $\mathbf{+ 6 0}$ \\
\hline Alk./Tox. & 855 & 16,5 & 323 & 12,9 & 532 & 19,8 & $\mathbf{+ 5 3 , 2}$ \\
\hline Alk. & 808 & 15,6 & 541 & 21,6 & 267 & 9,9 & $\mathbf{- 5 4 , 1}$ \\
\hline Sonstiges & 624 & 12 & 235 & 9,4 & 389 & 14,5 & $\mathbf{+ 5 4}$ \\
\hline Histo. & 398 & 7,7 & 312 & 12,5 & 86 & 3,2 & $-\mathbf{7 4 , 4}$ \\
\hline Alk./Histo./Tox. & 282 & 5,4 & 210 & 8,4 & 72 & 2,7 & $\mathbf{- 6 8 , 1}$ \\
\hline Tox. & 250 & 4,8 & 121 & 4,8 & 129 & 4,8 & $-\mathbf{0 , 8}$ \\
\hline Alk./Histo./ & 106 & 2 & 102 & 4,1 & 4 & 0,1 & $\mathbf{- 9 6 , 4}$ \\
\hline Histo./Neuropath. & 61 & 1,2 & 0 & 0 & 61 & 2,3 & - \\
\hline Neuropath. & 19 & 0,4 & 0 & 0 & 19 & 0,7 & - \\
\hline Gesamt & $\mathbf{5 . 1 9 0}$ & 100 & $\mathbf{2 . 5 0 1}$ & 100 & $\mathbf{2 . 6 8 9}$ & 100 & \\
\hline
\end{tabular}

Tab. 6: Basis der Todesursachendiagnostik 1969-1978 und 1998-2007 


\subsubsection{Pflegezustand}

Abbildung 12 zeigt die Verteilung des Pflegezustandes zum Obduktionszeitpunkt.

In 4.223 Fällen konnten Informationen zum Pflegezustand erhoben werden. $95 \%$ $(n=4.013, \Delta=+1,4 \%)$ dieser Fälle wurden als „unauffällig“ bewertet. Zeichen für Vernachlässigung wurden in 172 Fällen festgestellt ( $\Delta=-41,4 \%)$. Gesondert wurden Druckgeschwüre (Dekubitus) dokumentiert. So ließen sich die Informationen über die Dekubitus ersten Grades in 0,2 \% ( $n=10, \Delta=+142,7 \%)$, die Dekubitus zweiten Grades in 0,5\% $(n=21, \Delta=+160 \%)$ sowie die Dekubitus dritten Grades in 0,1\% $(\mathrm{n}=6, \Delta=+420 \%)$ aller Fälle erheben. Ein Dekubitus vierten Grades wurde in einem Fall (1969-1978) dokumentiert.

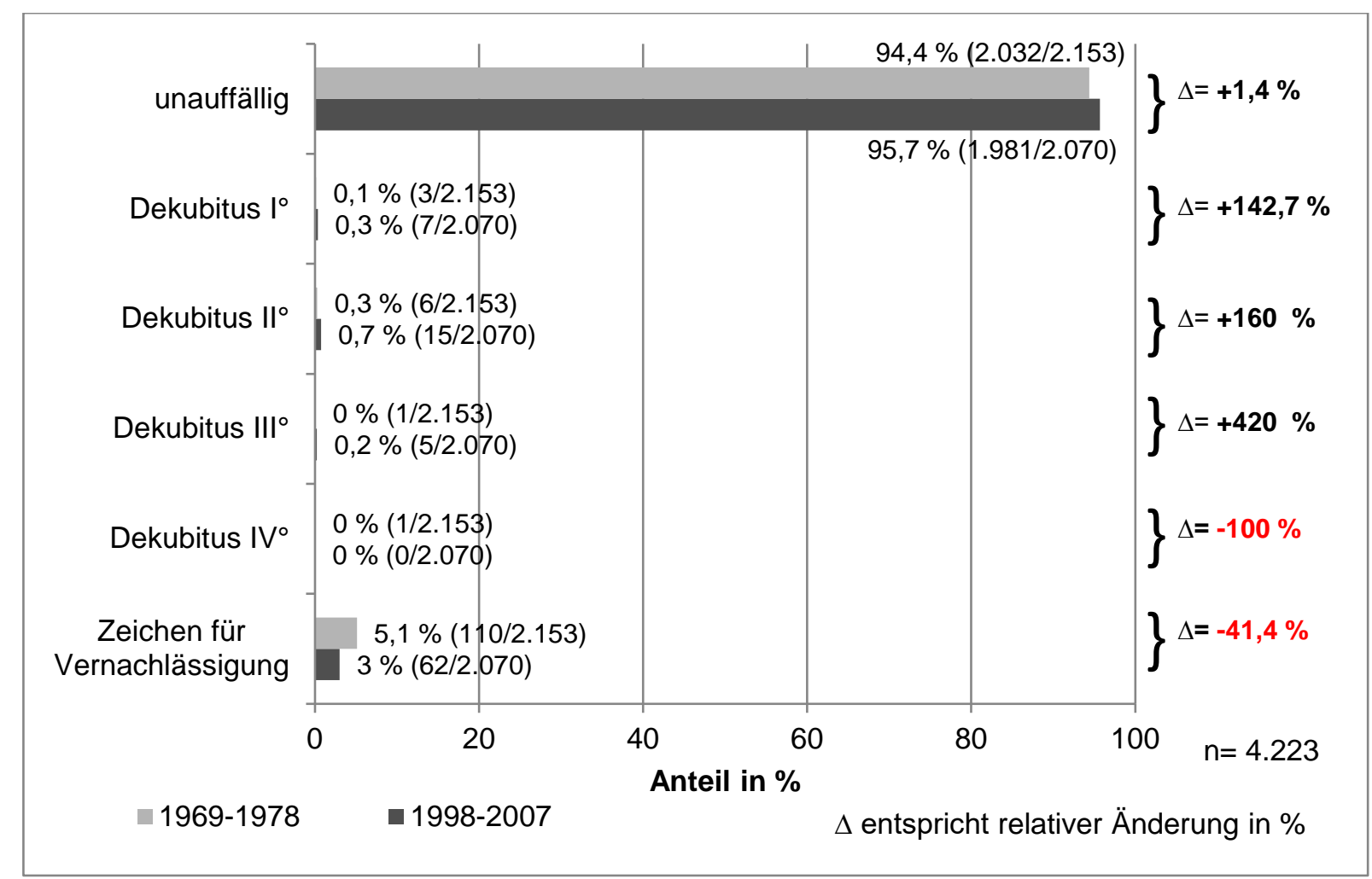

Abb. 12: Pflegezustand 1969-1978 und 1998-2007 


\subsection{Natürliche Todesursachen}

Die Verteilung der natürlichen Todesursachen gibt Tabelle 7 wieder.

Bei Betrachtung der Statistik im natürlichen Todesursachenspektrum fanden sich 1.733 Eintragungen. Die angegebene Zahl übersteigt die tatsächliche Zahl natürlicher Todesfälle, da in einigen Fällen das Zusammenspiel mehrerer Erkrankungen für das Sterbegeschehen eine Rolle spielte.

Herz-Kreislauf-Erkrankungen waren mit großem Abstand führend in dieser Kategorie. Insgesamt verstarben 748 Menschen am Herztod $(\Delta=+69,8 \%)$. Die zweithäufigste Ursache waren Erkrankungen des Respirationstraktes $(n=422)$, wo sich ein relativer Rückgang um 84,6 \% feststellen ließ. Von den 422 Fällen konnten 394 als Infektionen der Atemwege (Pneumonien, Bronchitiden sowie Kombinationen) kategorisiert werden, von denen 346 im Zeitraum von 1969 bis 1978 sowie lediglich 48 zwischen 1998 und 2007 erfasst wurden. Besonders deutlich war der Rückgang bei Säuglingen und Kleinkindern (bis einschließlich 5. Lebensjahr): Von 256 Kindertodesfällen (1969-1978) mit natürlicher Todesart wurde in 228 Fällen ein Atemwegsinfekt als todesursächlich dokumentiert. In den Jahren 1998-2007 waren es lediglich 9 Fälle bei 136 Verstorbenen (nicht abgebildet).

Mit einer Gesamtzahl von 154 Fällen $(\Delta=+41,6 \%)$ waren Erkrankungen des zentralen Nervensystems an dritter Stelle vertreten. Der relative Anstieg dieser Gruppe betrug 41,6\%. Weiterhin konnten 134 Todesfälle mit einer Erkrankung des Gastrointestinaltraktes in Verbindung gebracht werden, was einem relativen Anstieg von $126,5 \%$ entspricht. Infektionserkrankungen (systemische bzw. polylokale Infekte) lagen in 71 Fällen vor ( $\Delta=-45,8 \%)$. Angestiegen sind die Zahlen tödlicher Embolien ( $n=49, \Delta=+156,5 \%)$, tumorbedingter Todesfälle $(n=42, \Delta=+174,5 \%)$, innerer Blutungen $(n=42, \Delta=+515,6 \%)$, endokriner Erkrankungen $(n=24$, $\Delta=+102 \%)$, Stoffwechselentgleisungen $(\mathrm{n}=21, \Delta=+108 \%)$ sowie Erkrankungen des Urogenitaltraktes $(n=17, \Delta=+99,7 \%)$. Bei den Fruchttodesfällen $(n=8)$ wurde ein relativer Rückgang von $16,8 \%$ festgestellt. 


\begin{tabular}{|c|c|c|c|c|c|c|c|}
\hline & \multirow{2}{*}{\begin{tabular}{|l|} 
Gesamt \\
Anzahl
\end{tabular}} & \multirow[b]{2}{*}{ in $\%$} & \multicolumn{2}{|c|}{ 1969-1978 } & \multicolumn{2}{|c|}{ 1998-2007 } & \multirow{2}{*}{$\begin{array}{c}\text { rel. Änderung } \\
\text { in } \%\end{array}$} \\
\hline & & & Anzahl & in $\%$ & Anzahl & in $\%$ & \\
\hline Herz-Kreislauf-System & 748 & 43,2 & 246 & 31,3 & 502 & 53 & $+69,8$ \\
\hline Respirationstrakt & 422 & 24,3 & 356 & 45,2 & 66 & 7 & $-84,6$ \\
\hline ZNS-Erkrankungen & 154 & 8,9 & 57 & 7,2 & 97 & 10,2 & $+41,6$ \\
\hline Gastrointestinaltrakt & 134 & 7,7 & 36 & 4,6 & 98 & 10,4 & $+126,5$ \\
\hline Infektion & 71 & 4,1 & 43 & 5,5 & 28 & 3 & $-45,8$ \\
\hline tödliche Embolien & 49 & 2,8 & 12 & 1,5 & 37 & 3,9 & $+156,5$ \\
\hline Tumoren & 43 & 2,5 & 10 & 1,3 & 33 & 3,5 & $+174,5$ \\
\hline Verbluten & 42 & 2,4 & 5 & 0,6 & 37 & 3,9 & $+515,6$ \\
\hline endokrine Erkrankungen & 24 & 1,4 & 7 & 0,9 & 17 & 1,8 & +102 \\
\hline Stoffwechselentgleisung & 21 & 1,2 & 6 & 0,8 & 15 & 1,6 & +108 \\
\hline Urogenitaltrakt & 17 & 1 & 5 & 0,6 & 12 & 1,3 & $+99,7$ \\
\hline Fruchttod & 8 & 0,5 & 4 & 0,5 & 4 & 0,4 & $-16,8$ \\
\hline Gesamt & 1.733 & 100 & 787 & 100 & 946 & 100 & \\
\hline
\end{tabular}

Tab. 7: Natürliche Todesursachen 1969-1978 und 1998-2007 


\subsection{Nicht natürliche Todesursachen/-umstände}

\subsubsection{Formen der Gewalteinwirkung}

Die dokumentierten Gewalteinwirkungen sind in Tabelle 8 aufgeführt.

Bezogen auf alle Gewalteinwirkungen war die stumpfe Gewalt $(n=885)$ am häufigsten vertreten, gefolgt von der Gruppe „Intoxikation“ mit 791 Dokumentationen. Am dritthäufigsten ( $n=550)$ wurden Eintragungen aus der Gruppe „Ersticken/ Ertrinken“ erhoben. In der Gruppe „sonstiges Ersticken“ wurden insgesamt 112 Fälle erfasst (z.B. Aspiration von Blut, Fremdkörper oder Mageninhalt, Thoraxkompression, Verlegung der Atemwege etc.). Die Gruppe „sonstige Einwirkung“ enthielt die Gewalteinwirkungen chemische Noxen, Verhungern/Verdursten und Bolustod.

\begin{tabular}{|c|c|c|c|c|c|c|c|}
\cline { 2 - 9 } \multicolumn{1}{c|}{} & Gesamt & & $\mathbf{1 9 6 9 - 1 9 7 8}$ & & $\mathbf{1 9 9 8 - 2 0 0 7}$ & & rel. Änderung \\
\cline { 2 - 9 } \multicolumn{1}{c|}{} & Anzahl & in \% & Anzahl & in \% & Anzahl & in \% & in \% \\
\hline Schuss & $\mathbf{2 1 2}$ & 7,4 & $\mathbf{1 0 9}$ & 7,3 & $\mathbf{1 0 3}$ & 7,6 & $\mathbf{+ 5}$ \\
\hline scharfe Gewalt & & & & & & & \\
\hline Schnitt & 28 & 1 & 10 & 0,7 & 18 & 1,3 & $\mathbf{+ 1 0 0}$ \\
\hline Stich & 93 & 3,2 & 63 & 4,2 & 30 & 2,2 & $-47,1$ \\
\hline Kombination & 51 & 1,8 & 22 & 1,4 & 29 & 2,2 & $\mathbf{+ 4 6 , 5}$ \\
\hline Gesamt & $\mathbf{1 7 2}$ & 6 & $\mathbf{9 5}$ & 6,3 & $\mathbf{7 7}$ & 5,7 & $\mathbf{- 9 , 9}$ \\
\hline halbscharf Gewalt & $\mathbf{2 8}$ & 1 & $\mathbf{2 4}$ & 1,6 & $\mathbf{4}$ & 0,3 & $\mathbf{- 8 1 , 5}$ \\
\hline stumpfe Gewalt & $\mathbf{8 8 5}$ & 31,1 & $\mathbf{5 0 3}$ & 33,6 & $\mathbf{3 8 2}$ & $\mathbf{2 8 , 3}$ & $\mathbf{- 1 5 , 6}$ \\
\hline Ersticken/Ertrinken & & & & & & & \\
\hline Strangulation & 200 & 7 & 89 & 5,9 & 111 & 8,2 & $\mathbf{+ 3 8 , 6}$ \\
\hline sonstiges Ersticken & 112 & 3,9 & 75 & 5 & 37 & 2,7 & $-\mathbf{4 5 , 2}$ \\
\hline Ertrinken & 238 & 8,4 & 136 & 9,1 & 102 & 7,6 & $\mathbf{- 1 6 , 7}$ \\
\hline Gesamt & $\mathbf{5 5 0}$ & 19,3 & $\mathbf{3 0 0}$ & 20 & $\mathbf{2 5 0}$ & 18,5 & $\mathbf{- 7 , 4}$ \\
\hline Stromtod & $\mathbf{2 6}$ & 0,9 & $\mathbf{1 5}$ & 1 & $\mathbf{1 1}$ & 0,8 & $\mathbf{- 1 8 , 5}$ \\
\hline thermische Schädigung & & & & & & & \\
\hline Hitzeeinwirkung & 125 & 4,4 & 66 & 4,4 & 59 & 4,4 & $\mathbf{- 0 , 7}$ \\
\hline Kälteeinwirkung & 34 & 1,2 & 17 & 1,1 & 17 & 1,3 & $\mathbf{+ 1 1 , 1}$ \\
\hline Gesamt & $\mathbf{1 5 9}$ & 5,6 & $\mathbf{8 3}$ & 5,5 & $\mathbf{7 6}$ & 5,7 & $\mathbf{+ 1 , 7}$ \\
\hline Intoxikation & $\mathbf{7 9 1}$ & 27,8 & $\mathbf{3 5 3}$ & 23,6 & $\mathbf{4 3 8}$ & $\mathbf{3 2 , 5}$ & $\mathbf{+ 3 7 , 9}$ \\
\hline sonstige Einwirkung & $\mathbf{2 5}$ & 0,9 & $\mathbf{1 7}$ & 1,1 & $\mathbf{8}$ & 0,6 & $\mathbf{- 4 7 , 7}$ \\
\hline Gesamt & $\mathbf{2 . 8 4 8}$ & $\mathbf{1 0 0}$ & $\mathbf{1 1 4 9 9}$ & $\mathbf{1 0 0}$ & $\mathbf{1 . 3 4 9}$ & $\mathbf{1 0 0}$ & \\
\hline
\end{tabular}

Tab. 8: Formen der Gewalteinwirkung 1969-1978 und 1998-2007 


\subsubsection{Anatomische Lokalisation bei stumpfer Gewalteinwirkung}

Die Einwirkung stumpfer Gewalt und ihre Folgen (z.B. Subarachnoidalblutung, Schädel-Hirn-Trauma, Abdominaltrauma, Extremitätenabriss etc.) wurden der anatomischen Lokalisation entsprechend in folgender Tabelle (Tab. 9) eingeordnet.

\begin{tabular}{|l|c|c|c|c|c|c|c|}
\cline { 2 - 9 } \multicolumn{1}{c|}{} & Gesamt & & $\mathbf{1 9 6 9 - 1 9 7 8}$ & & $\mathbf{1 9 9 8 - 2 0 0 7}$ & & rel. Änderung \\
\cline { 2 - 9 } \multicolumn{1}{c|}{} & Anzahl & in \% & Anzahl & in \% & Anzahl & in \% & in \% \\
\hline ZNS & 569 & 64,3 & 379 & 75,3 & 190 & 49,7 & $\mathbf{- 3 4}$ \\
\hline Rumpf & 114 & 12,9 & 88 & 17,5 & 26 & 6,8 & $-61,1$ \\
\hline Kombinationen/Polytrauma & 198 & 22,4 & 32 & 6,4 & 166 & 43,5 & $\mathbf{+ 5 8 3 , 1}$ \\
\hline Gesamt & 4 & 0,4 & 4 & 0,8 & 0 & 0 & $\mathbf{- 1 0 0}$ \\
\hline
\end{tabular}

Tab. 9: Anatomische Lokalisation bei stumpfer Gewalteinwirkung 1969-1978 und 1998-2007 


\subsubsection{Verkehrsteilnehmer}

Die Verteilung der verschiedenen Verkehrsteilnehmer ist in Abbildung 13 aufgeführt. Die Zahl der Dokumentationen $(n=594)$ übersteigt die der (unter 3.1.12.) klassifizierten Verkehrsunfälle $(n=551)$, da in einigen Fällen bei den Protokollen ein Kausalzusammenhang zwischen der Verkehrsteilnahme und dem Todeseintritt nicht eindeutig war, die Verkehrsteilnahme aber dennoch dokumentiert wurde. Ebenfalls gab es Fälle von Suiziden bzw. Tötungsdelikten im Straßenverkehr (entsprechend wurde bei „Todesart/Kategorie nach Obduktion“ die Verkehrsteilnahme nicht erfasst, sondern der übergeordnete Todesumstand). Des Weiteren entzogen sich einige wenige Fälle der Klassifizierung als „typischen Verkehrsunfall“, jedoch wurde auch hier die Art der Verkehrsteilnahme dokumentiert (z.B. als „tödlich verunglückte Pferdekutscheninsassen“ oder „Fallschirmspringer"). Insgesamt wurden im ersten Zeitraum (1969-1978) 368 Eingaben und im zweiten (1998-2007) 226 Eingaben erhoben. Die Fußgänger stehen an erster Stelle $(n=251,42,3 \%, \Delta=-21 \%)$, es folgen Pkw-Fahrer ( $n=88,14,8 \%, \Delta=+29,6 \%)$ und Pkw-Insassen ( $n=81,13,6 \%$, $\Delta=+36,9 \%)$.

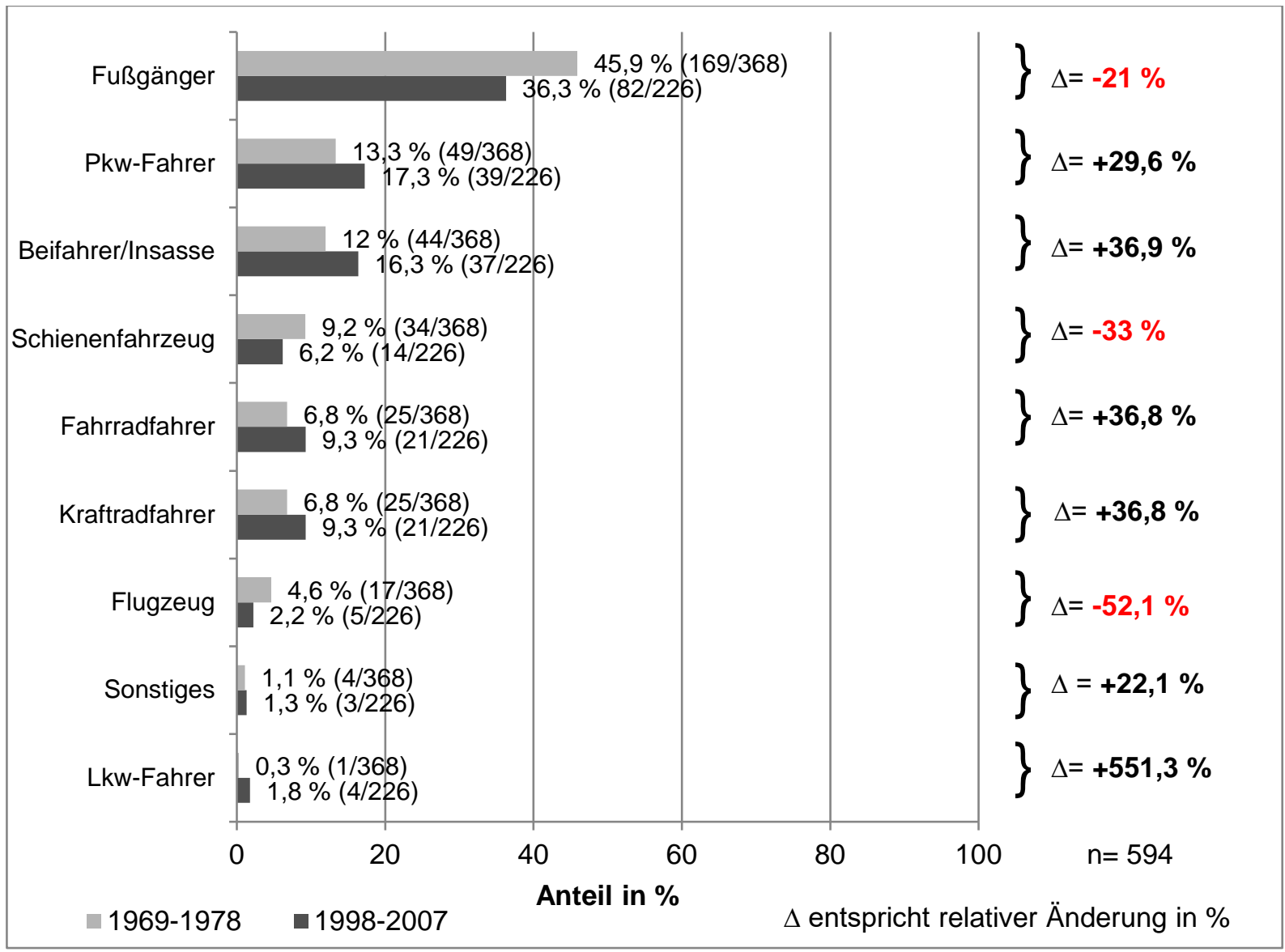

Abb. 13: Verkehrsteilnehmer 1969-1978 und 1998-2008 


\subsubsection{Sturz}

Die Verteilung der sturzbedingten Todesfälle ist Abbildung $14 \mathrm{zu}$ entnehmen. Insgesamt hat die Zahl der Fälle von 120 (1969-1978) auf 143 (1998-2007) zugenommen. 8 Fälle (6 Stürze aus einem Bett, ein Sturz auf einen Heizungskörper und ein Mehrfachsturz) wurden der Gruppe „Sonstiges“ zugeordnet.

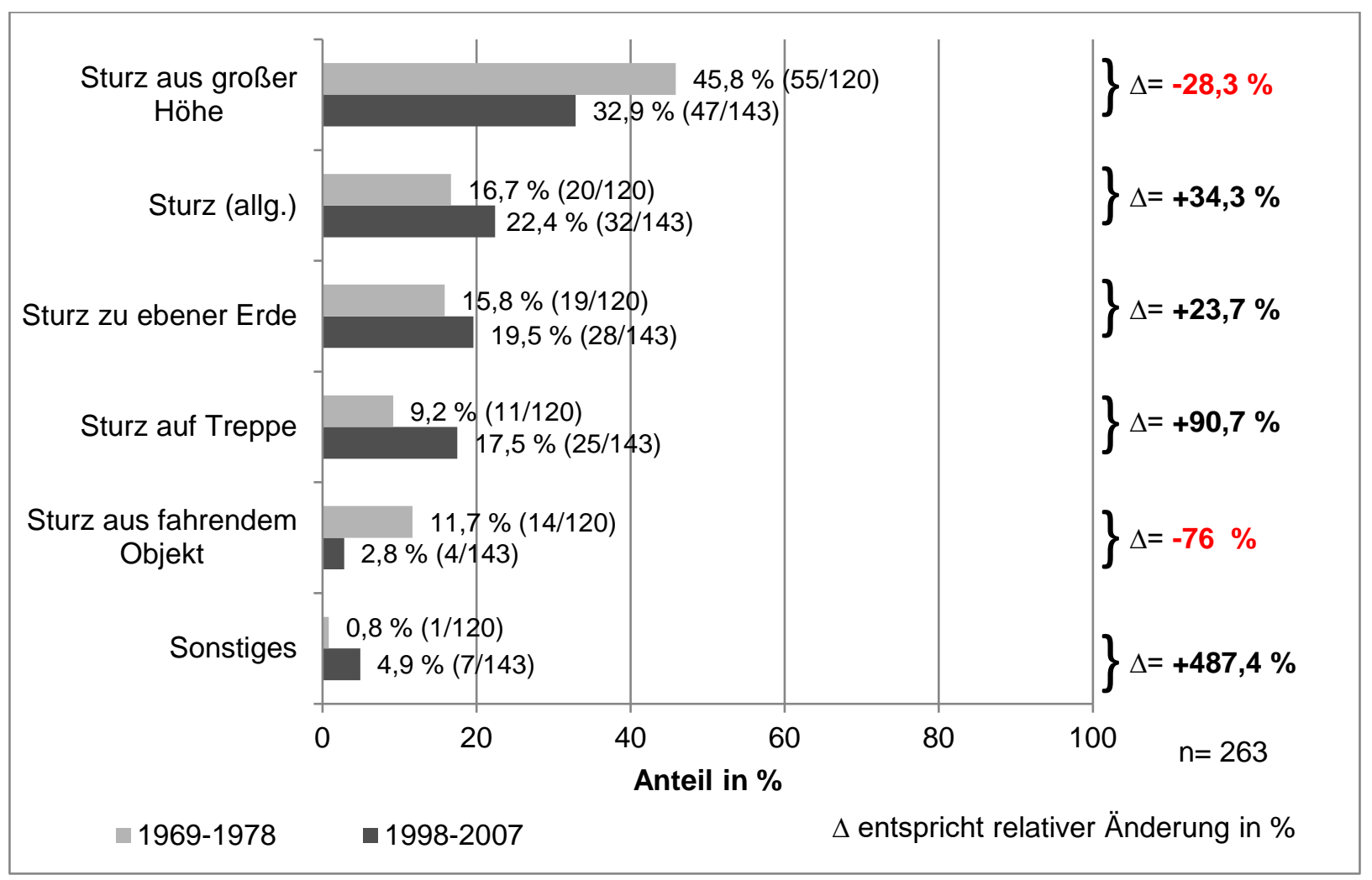

Abb. 14: Sturz 1969-1978 und 1998-2007 


\subsubsection{Schussverletzung}

\subsubsection{Schussart}

Die Verteilung der Schussarten ist der Abbildung $15 \mathrm{zu}$ entnehmen. In den Jahren von 1969-1978 konnten Befunde von 107 Steckschüssen an 52 Verstorbenen sowie 105 Durchschüssen an 34 Verstorbenen festgestellt werden. Im zweiten Untersuchungszeitraum (1998-2007) wurden 53 Steckschussbefunde an 33 Verstorbenen sowie 82 Durchschussbefunde an 58 Verstorbenen erhoben. Ferner lagen in 16 (1969-1978) und 10 (1998-2007) Fällen Kombinationen aus Steck- und Durchschüssen vor. In 9 Fällen wurde auf Angaben zur Schussart verzichtet bzw. waren solche nicht eruierbar.

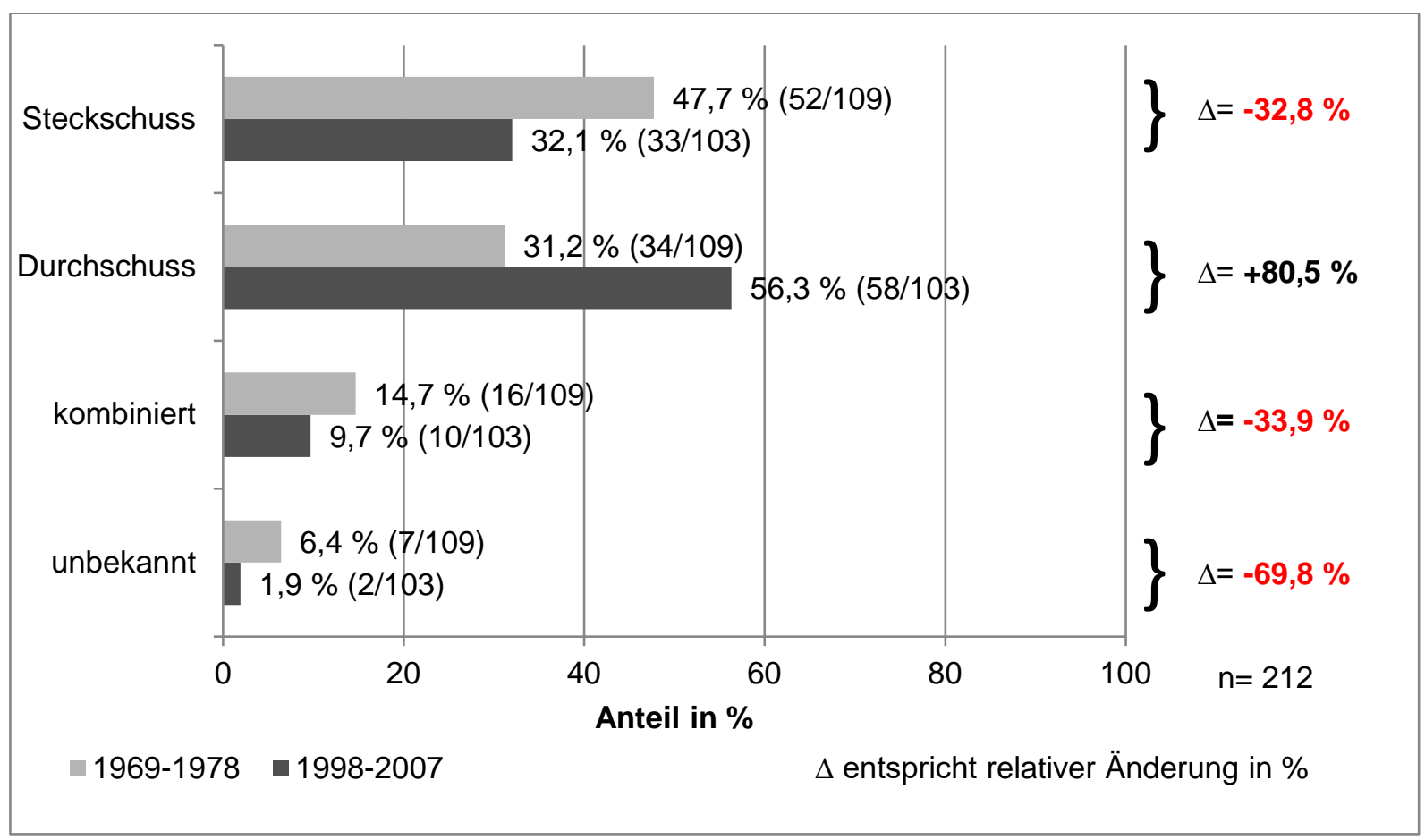

Abb. 15: Schussart 1969-1978 und 1998-2007 


\subsubsection{Tatwaffe (Schuss- bzw. Explosionseinwirkung)}

Die Häufigkeit der verwendeten Schusswaffen bzw. Explosionseinwirkung ist der Tabelle $10 \mathrm{zu}$ entnehmen. Nicht näher bezeichnete Schusswaffen wurden der Gruppe „unbekannt“ zugeordnet. Eine als Kugelschreiber umgebaute Schusswaffe wurde als „Sonstiges“ dokumentiert.

\begin{tabular}{|l|c|c|c|c|c|c|c|}
\cline { 2 - 8 } \multicolumn{1}{c|}{} & Gesamt & & $\mathbf{1 9 6 9 - 1 9 7 8}$ & & $\mathbf{1 9 9 8 - 2 0 0 7}$ & & rel. Änderung \\
\cline { 2 - 8 } \multicolumn{1}{c|}{} & Anzahl & in \% & Anzahl & in \% & Anzahl & in \% & in \% \\
\hline Pistole & 89 & 44 & 40 & 37,4 & 49 & 51,6 & $\mathbf{+ 3 8}$ \\
\hline Gewehr & 46 & 22,8 & 36 & 33,6 & 10 & 10,5 & $-68,7$ \\
\hline unbekannt & 36 & 17,8 & 10 & 9,4 & 26 & 27,3 & $\mathbf{+ 1 9 2 , 8}$ \\
\hline Revolver & 22 & 10,9 & 15 & 14 & 7 & 7,3 & $-47,4$ \\
\hline Explosion & 6 & 3 & 5 & 4,7 & 1 & 1,1 & $\mathbf{- 7 7 , 5}$ \\
\hline Bolzenschussapparat & 2 & 1 & 1 & 0,9 & 1 & 1,1 & $\mathbf{+ 1 2 , 6}$ \\
\hline Sonstiges & 1 & 0,5 & 0 & 0 & 1 & 1,1 & \\
\hline Gesamt & $\mathbf{2 0 2}$ & 100 & $\mathbf{1 0 7}$ & 100 & $\mathbf{9 5}$ & 100 & \\
\hline
\end{tabular}

Tab. 10: Tatwaffe (Schuss- bzw. Explosionseinwirkung) 1969-1978 und 1998-2007

\subsubsection{Erhängen/Strangulation}

Die Verteilung der Gewalteinwirkungen gegen den Hals ist Tabelle $11 \mathrm{zu}$ entnehmen. Insgesamt stieg die Zahl der Todesfälle durch Erhängen von 5 auf 64 an. Todesfälle durch Erdrosselung waren mit einer Gesamtzahl von 58 vertreten $(\Delta=-51 \%)$, gefolgt von der Gewalteinwirkung „Erwürgen“ mit 52 Fällen $(\Delta=-75,9 \%)$.

\begin{tabular}{|l|c|c|c|c|c|c|c|}
\cline { 2 - 9 } \multicolumn{1}{c|}{} & Gesamt & & $\mathbf{1 9 6 9 - 1 9 7 8}$ & & $\mathbf{1 9 9 8 - 2 0 0 7}$ & rel. Änderung \\
\cline { 2 - 9 } \multicolumn{1}{l|}{} & Anzahl & in \% & Anzahl & in \% & Anzahl & in \% & in \% \\
\hline Erdrosseln & 58 & 29 & 36 & 40,5 & 22 & 19,8 & -51 \\
\hline Erwürgen & 52 & 26 & 40 & 44,9 & 12 & 10,8 & $-75,9$ \\
\hline $\begin{array}{l}\text { Erhängen - atypisch } \\
\text { unbekannt) }\end{array}$ & 45 & 22,5 & 4 & 4,5 & 41 & 37 & $\mathbf{+ 7 2 1 , 8}$ \\
\hline Erhängen - typisch & 14 & 7 & 0 & 0 & 14 & 12,6 & \\
\hline Kombinationen & 10 & 5 & 1 & 1,1 & 9 & 8,1 & $\mathbf{+ 6 2 1 , 6}$ \\
\hline sonstige Halskompression & 13 & 6,5 & 2 & 2,3 & 11 & 9,9 & $\mathbf{- 7 3 , 3}$ \\
\hline Gesamt: & $\mathbf{2 0 0}$ & 100 & $\mathbf{8 9}$ & 100 & $\mathbf{1 1 1}$ & 100 & $\mathbf{4 3 1}$ \\
\hline
\end{tabular}

Tab. 11: Erhängen/Strangulation 1969-1978 und 1998-2007 


\subsubsection{Ersticken}

Die Verteilung des Erstickens ist in Abbildung 16 dargestellt.

Insgesamt hat sich die Zahl der in dieser Gruppe erfassten Fälle von 75 (1969-1978) auf 37 (1998-2007) nahezu halbiert.

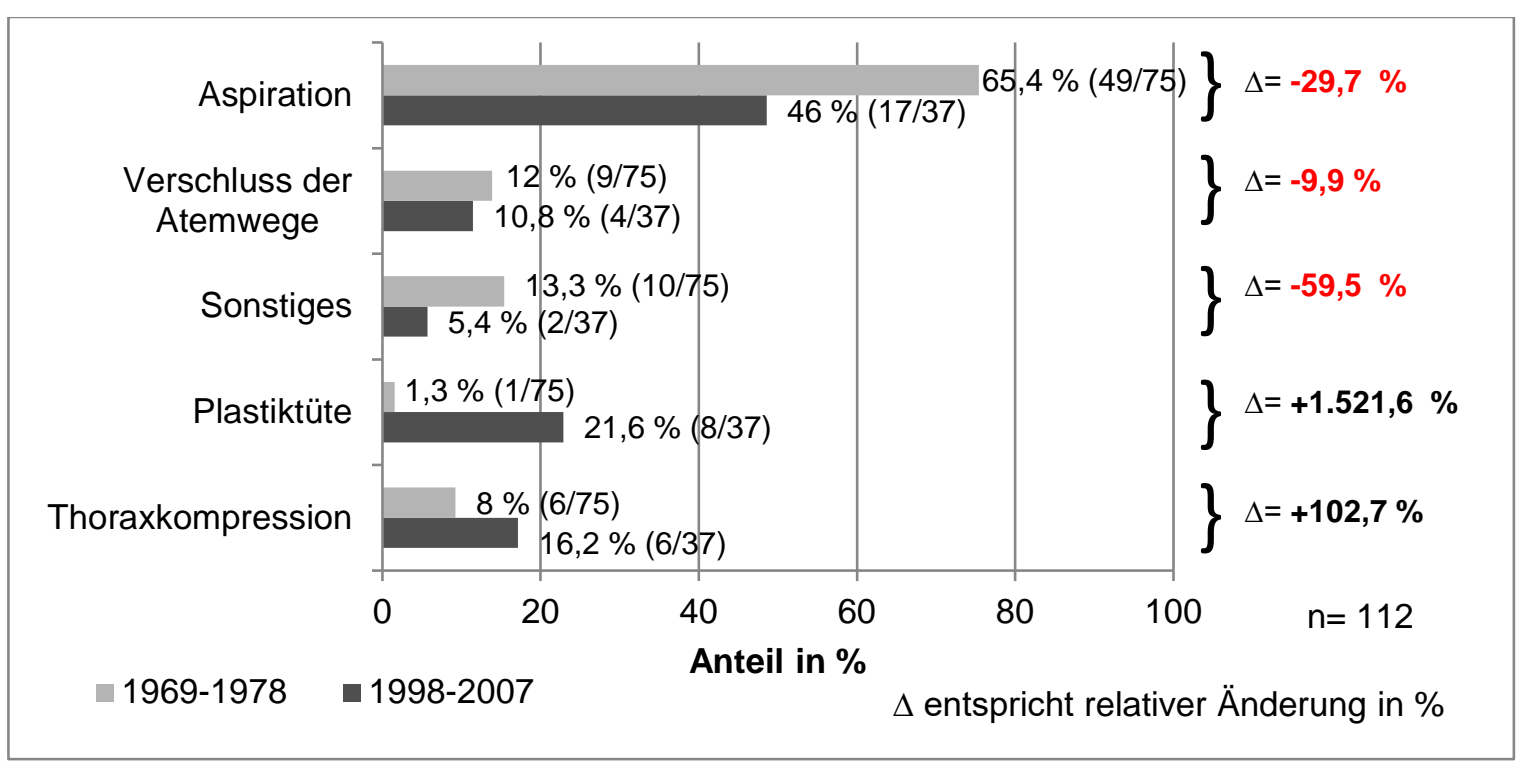

Abb. 16: Ersticken 1969-1978 und 1998-2007

\subsubsection{Ertrinken/Tod im Wasser}

In Tabelle 12 ist die Verteilung von Todesfällen im Wasser bzw. durch Ertrinken wiedergegeben, ebenso Fälle, bei denen von einem mutmaßlichen Ertrinken $(n=3)$ ausgegangen wurde, sowie ein Leichenfall, der im Wasser entsorgt wurde.

\begin{tabular}{|c|c|c|c|c|c|c|c|}
\hline & Gesamt & & 1969-1978 & & 1998-2007 & & rel. Änderung \\
\hline & Anzahl & in \% & Anzahl & in $\%$ & Anzahl & in $\%$ & in $\%$ \\
\hline Ertrinken & 185 & 77,7 & 101 & 74,3 & 84 & 82,3 & $+10,9$ \\
\hline Badewanne & 29 & 12,2 & 14 & 10,3 & 15 & 14,7 & $+42,9$ \\
\hline sog. Badetod & 14 & 5,9 & 13 & 9,6 & 1 & 1 & $-89,7$ \\
\hline Tod nach Beinahe-Ertrinken & 3 & 1,3 & 2 & 1,5 & 1 & 1 & $-33,3$ \\
\hline mutmaßliches Ertrinken & 3 & 1,3 & 3 & 2,2 & 0 & 0 & -100 \\
\hline Sonstiges & 2 & 0,8 & 1 & 0,7 & 1 & 1 & $+33,3$ \\
\hline Entsorgung im Wasser & 1 & 0,4 & 1 & 0,7 & 0 & 0 & -100 \\
\hline Tauchunfall & 1 & 0,4 & 1 & 0,7 & 0 & 0 & -100 \\
\hline Gesamt & 238 & 100 & 136 & 100 & 102 & 100 & \\
\hline
\end{tabular}

Tab. 12: Ertrinken 1969-1978 und 1998-2007 


\subsubsection{Thermische Einwirkung}

\subsubsection{Hitzeeinwirkung}

Die Verteilung der Hitzeeinwirkung ist der Tabelle 13 zu entnehmen.

Die Zahl hitzebedingter Todesfälle betrug im gesamten Zeitraum 125 und war im Verlauf leicht rückläufig. Die Gruppe „Sonstiges“ enthielt Fälle, die den genannten Kategorien nicht eindeutig zuzuordnen waren bzw. Kombinationen.

\begin{tabular}{|l|c|c|c|c|c|c|c|}
\cline { 2 - 8 } \multicolumn{1}{c|}{} & Gesamt & & $\mathbf{1 9 6 9 - 1 9 7 8}$ & & $\mathbf{1 9 9 8 - 2 0 0 7}$ & & rel. Änderung \\
\cline { 2 - 9 } \multicolumn{1}{c|}{} & Anzahl & in \% & Anzahl & in \% & Anzahl & in \% & in \% \\
\hline Verbrennung allg. & 29 & 23,2 & 20 & 30,3 & 9 & 15,2 & $-49,7$ \\
\hline postmortales Verbrennen & 27 & 21,6 & 13 & 19,7 & 14 & 23,7 & $+\mathbf{2 0 , 5}$ \\
\hline Sonstiges/Kombinationen & 24 & 19,2 & 5 & 7,6 & 19 & 32,2 & $+\mathbf{3 2 5 , 1}$ \\
\hline Wohnungsbrand & 14 & 11,2 & 8 & 12,1 & 6 & 10,2 & $\mathbf{- 1 6 , 1}$ \\
\hline Spättodesfall & 13 & 10,4 & 10 & 15,2 & 3 & 5,1 & $-\mathbf{6 6 , 4}$ \\
\hline Autobrand & 12 & 9,6 & 7 & 10,6 & 5 & 8,5 & $\mathbf{- 2 0 , 1}$ \\
\hline Verbrühung & 5 & 4 & 2 & 3 & 3 & 5,1 & $+\mathbf{+ 6 7 , 8}$ \\
\hline Hitzschlag & 1 & 0,8 & 1 & 1,5 & 0 & 0 & $\mathbf{- 1 0 0}$ \\
\hline Gesamt & $\mathbf{1 2 5}$ & 100 & $\mathbf{6 6}$ & 100 & $\mathbf{5 9}$ & 100 & \\
\hline
\end{tabular}

Tab. 13: Thermische Einwirkung 1969-1978 und 1998-2007

\subsubsection{Erfrieren/Unterkühlung}

Die Tabelle 14 gibt einen Überblick über die Verteilung der Kategorie „Erfrieren/Unterkühlung“.

Im gesamten Untersuchungsgut $(n=5.190)$ fanden sich 34 Fälle, die unter „Erfrieren/ Unterkühlung“ erfasst wurden. Eine Unterkühlung konnte in 79,4\% der Fälle ( $n=27$, $\Delta=+45,5 \%)$ ermittelt werden. Bei den restlichen 7 Fällen $(20,6 \%, \Delta=-83,3 \%)$ war der Tod durch Erfrieren eingetreten.

\begin{tabular}{|l|c|c|c|c|c|c|c|}
\cline { 2 - 8 } \multicolumn{1}{c|}{} & Gesamt & & $\mathbf{1 9 6 9 - 1 9 7 8}$ & & $\mathbf{1 9 9 8 - 2 0 0 7}$ & & rel. Änderung \\
\cline { 2 - 8 } \multicolumn{1}{c|}{} & Anzahl & in \% & Anzahl & in \% & Anzahl & in \% & in \% \\
\hline Unterkühlung & 27 & 79,4 & 11 & 64,7 & 16 & 94,1 & $+\mathbf{4 5 , 5}$ \\
\hline Erfrieren & 7 & 20,6 & 6 & 35,3 & 1 & 5,9 & $-83,3$ \\
\hline Gesamt & $\mathbf{3 4}$ & 100 & $\mathbf{1 7}$ & 100 & $\mathbf{1 7}$ & 100 & \\
\hline
\end{tabular}

Tab. 14: Erfrieren/Unterkühlung 1969-1978 und 1998-2007 


\subsubsection{Stromtod}

Die Einwirkung von Elektrizität konnte in 26 Obduktionsfällen erfasst werden (Abb. 17). Die Dokumentation eines Todes durch Niederspannung wurde in 14 Fällen dokumentiert. In 7 Fällen wurde der Tod durch systemische Stromeinwirkung (z.B. durch Elektrogeräte in einer Badewanne) festgestellt.

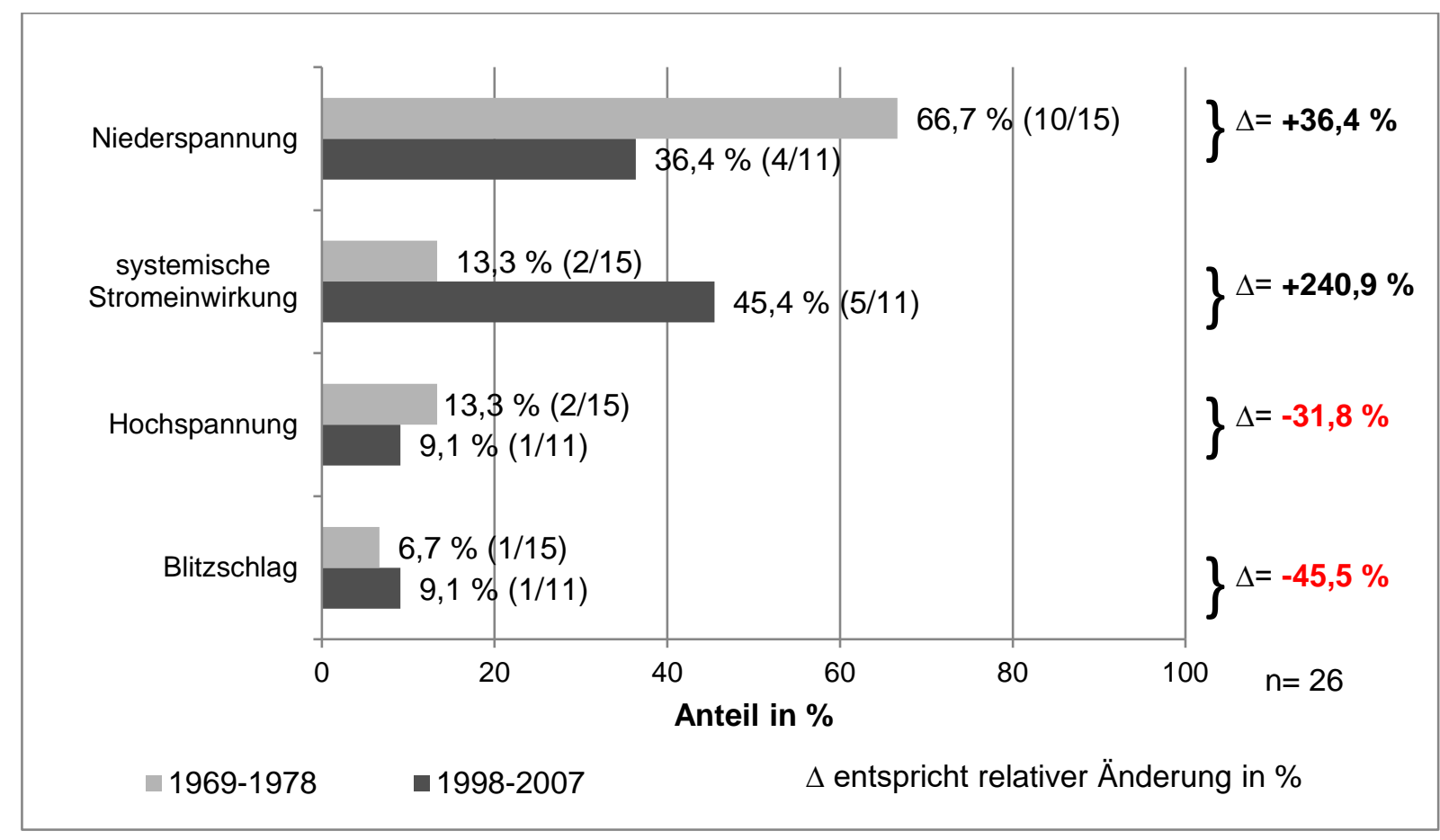

Abb. 17: Stromtod 1969-1978 und 1998-2007

\subsubsection{Sonstige Einwirkungen}

Die sonstigen Formen der Gewalteinwirkungen sind der Tabelle 15 zu entnehmen. Der Bolustod ( $n=11, \Delta=+77,1 \%$ ) stellt eine Ausnahme dar. Er entsteht durch einen reflektorischen Herz-Kreislauf-Stillstand aufgrund vagaler Reizung des KehlkopfNerven-Geflechts durch Fremdkörper.

\begin{tabular}{|l|c|c|c|c|c|c|c|}
\cline { 2 - 8 } \multicolumn{1}{c|}{} & Gesamt & & $\mathbf{1 9 6 9 - 1 9 7 8}$ & & $\mathbf{1 9 9 8 - 2 0 0 7}$ & & rel. Änderung \\
\cline { 2 - 9 } \multicolumn{1}{l|}{} & Anzahl & in \% & Anzahl & in \% & Anzahl & in \% & in \% \\
\hline Bolustod & 11 & 44 & 6 & 35,3 & 5 & 62,5 & $\mathbf{+ 7 7 , 1}$ \\
\hline Verhungern/Verdursten & 10 & 40 & 8 & 47,1 & 2 & 25 & $-\mathbf{4 6 , 9}$ \\
\hline Säure & 2 & 8 & 1 & 5,9 & 1 & 12,5 & $\mathbf{+ 1 1 2 , 5}$ \\
\hline Lauge & 2 & 8 & 2 & 11,7 & 0 & 0 & $\mathbf{- 1 0 0}$ \\
\hline Gesamt & $\mathbf{2 5}$ & 100 & $\mathbf{1 7}$ & 100 & $\mathbf{8}$ & 100 & \\
\hline
\end{tabular}

Tab. 15: Sonstige Einwirkung 1969-1978 und 1998-2007 


\subsubsection{Neugeborene/Föten}

Detailliertere Informationen zu der Kategorie „Neugeborene/Föten“ sind in Tabelle 16 wiedergegeben. Insgesamt war die Zahl der Obduktionsfälle in dieser Gruppe rückläufig. Fälle in Verbindung mit einem Schwangerschaftsabbruch sind vollständig zurückgegangen. Unter „Sonstiges“ wurde der Fall einer Nachsektion mit fraglichem Schwangerschaftsabbruch dokumentiert.

\begin{tabular}{|l|c|c|c|c|c|c|c|}
\cline { 2 - 8 } \multicolumn{1}{c|}{} & Gesamt & & $\mathbf{1 9 6 9 - 1 9 7 8}$ & & $\mathbf{1 9 9 8 - 2 0 0 7}$ & & rel. Änderung \\
\cline { 2 - 9 } \multicolumn{1}{c|}{} & Anzahl & in \% & Anzahl & in \% & Anzahl & in \% & in \% \\
\hline Gelebthaben bewiesen & 14 & 43,8 & 12 & 48 & 2 & 28,6 & $-40,5$ \\
\hline $\begin{array}{l}\text { Gelebthaben nicht } \\
\text { bewiesen oder Totgeburt }\end{array}$ & 10 & 31,2 & 6 & 24 & 4 & 57,1 & $+\mathbf{+ 1 3 8 , 1}$ \\
\hline Schwangerschaftsabbruch & 7 & 21,9 & 7 & 28 & 0 & 0 & -100 \\
\hline Sonstiges & 1 & 3,1 & 0 & 0 & 1 & 14,3 & \\
\hline Gesamt & $\mathbf{3 2}$ & 100 & $\mathbf{2 5}$ & 100 & $\mathbf{7}$ & 100 & \\
\hline
\end{tabular}

Tab. 16: Neugeborene/Föten 1969-1978 und 1998-2007 


\subsubsection{Tod nach medizinischen Maßnahmen}

Die Verteilung der Fälle, die in zeitlichem Zusammenhang mit einer ärztlichen Behandlungsmaßnahme standen, kann der Abbildung 18 entnommen werden.

Zugenommen haben Todesfälle während einer Operation („Mors in tabula“, $\Delta=+113,3 \%)$ und nach einer Operation bzw. nach einem medizinischen Eingriff $(\Delta=+31 \%)$. Rückläufig waren Narkosezwischenfälle ( $\Delta=-83,3 \%)$, Todesfälle nach Medikation ( $\Delta=-61,9 \%)$ und Fälle, die der Gruppe „Sonstiges“ zugeordnet wurden $(\Delta=-55,6 \%)$.

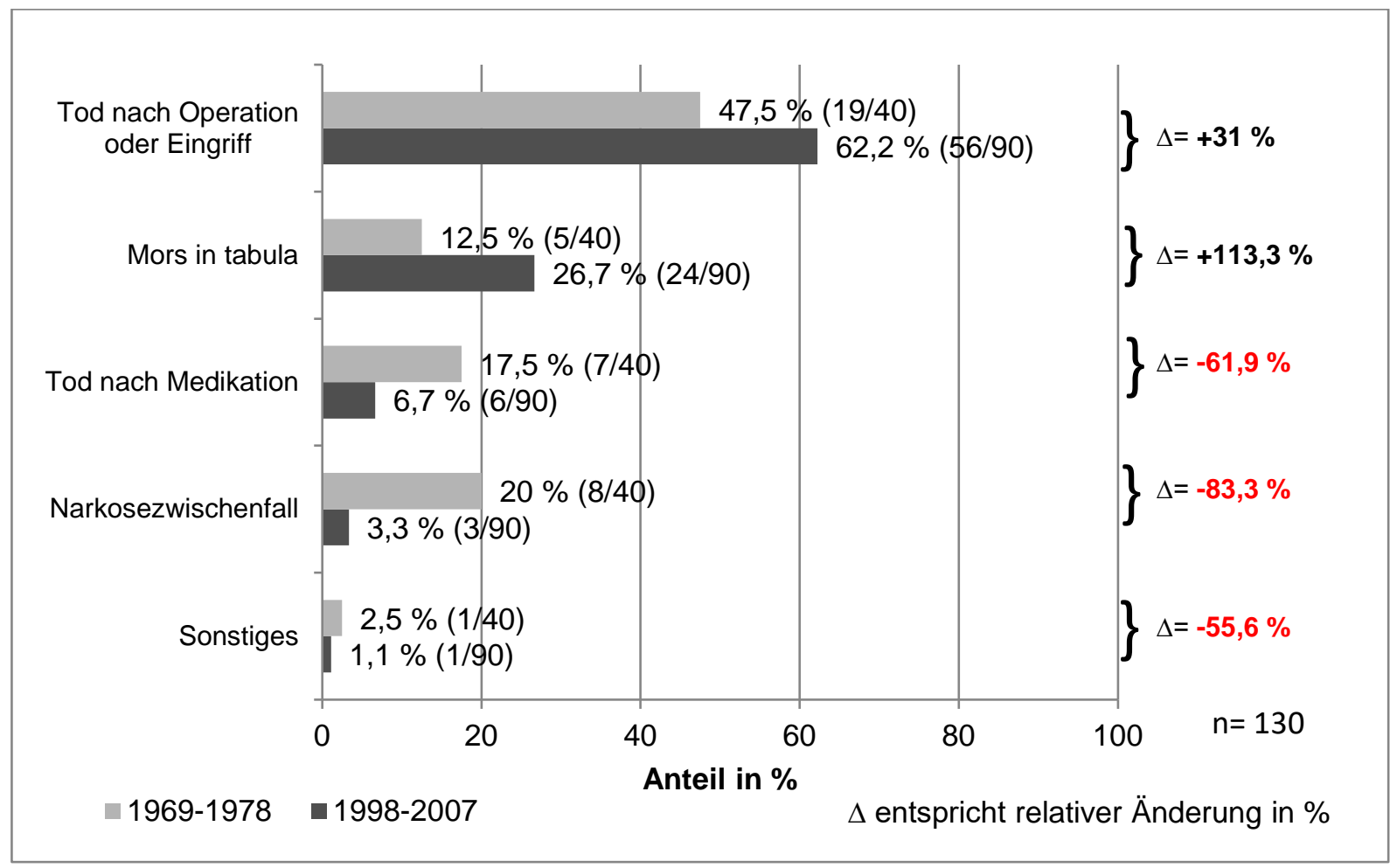

Abb. 18: Tod nach medizinischen Maßnahmen 1969-1978 und 1998-2007 


\subsubsection{Systemische bzw. direkte Todesursachen}

Die Verteilung der Todesursachen, die systemisch bzw. direkt bedingt waren, sind der Tabelle 17 zu entnehmen.

Die häufigste Todesursache in dieser Gruppe war das „zentrale Regulationsversagen“ mit insgesamt 557 Fällen ( $\Delta=-17,7 \%)$, gefolgt von der Kategorie „Polytrauma“ ( $\mathrm{n}=163, \Delta=+1.040,9 \%)$ und dem „Verbluten nach innen“ ( $\mathrm{n}=141$, $\Delta=-59 \%)$. Die Gruppe „Sonstiges“ enthielt Fälle, die den übrigen Kategorien nicht sicher zuzuordnen waren.

\begin{tabular}{|l|c|c|c|c|c|c|c|}
\cline { 2 - 8 } \multicolumn{1}{l|}{} & Gesamt & & $\mathbf{1 9 6 9 - 1 9 7 8}$ & & $\mathbf{1 9 9 8 - 2 0 0 7}$ & & rel. Änderung \\
\cline { 2 - 9 } & Anzahl & in \% & Anzahl & in \% & Anzahl & in \% & in \% \\
\hline zentrales Regulationsversagen & 557 & 48 & 335 & 52,2 & 222 & 42,9 & $\mathbf{- 1 7 , 7}$ \\
\hline Polytrauma & 163 & 14,1 & 16 & 2,5 & 147 & 28,4 & $\mathbf{+ 1 . 0 4 0 , 9}$ \\
\hline Verbluten nach innen & 141 & 12,2 & 106 & 16,5 & 35 & 6,8 & $-\mathbf{5 9}$ \\
\hline Kombinationen & 96 & 8,3 & 76 & 11,8 & 20 & 3,9 & $-\mathbf{6 7 , 3}$ \\
\hline Verbluten nach außen & 62 & 5,3 & 29 & 4,5 & 33 & 6,4 & $\mathbf{+ 4 1 , 3}$ \\
\hline Verbluten (innen \& außen) & 45 & 3,9 & 23 & 3,6 & 22 & 4,3 & $\mathbf{+ 1 8 , 8}$ \\
\hline Organruptur(en) & 44 & 3,8 & 29 & 4,5 & 15 & 2,9 & $\mathbf{- 3 5 , 8}$ \\
\hline Herzbeuteltamponade & 21 & 1,8 & 14 & 2,2 & 7 & 1,3 & $\mathbf{- 3 7 , 9}$ \\
\hline Sonstiges & 30 & 2,6 & 14 & 2,2 & 16 & 3,1 & $\mathbf{+ 4 1 , 9}$ \\
\hline Gesamt & $\mathbf{1 . 1 5 9}$ & 100 & $\mathbf{6 4 2}$ & 100 & $\mathbf{5 1 7}$ & 100 & \\
\hline
\end{tabular}

Tab. 17: Systemische bzw. direkte Todesursachen 1969-1978 und 1998-2007 


\subsubsection{Mittelbare Todesursachen}

Die Häufigkeiten mittelbarer Todesursachen sind in Tabelle 18 dargestellt. Rückläufig waren posttraumatische Pneumonien ( $\mathrm{n}=98, \Delta=-57,2 \%)$, Lungenembolien ( $\mathrm{n}=54$, $\Delta=-25,1 \%)$ sowie Fettembolien $(\mathrm{n}=18, \Delta=-31,6 \%)$. Zugenommen haben septische Geschehen ( $n=34, \Delta=+1.233,3 \%)$, Multiorganversagen $(n=52, \Delta=+52,4 \%)$ und die Gruppe der „Sonstigen“ ( $n=29, \Delta=+190,9 \%)$.

\begin{tabular}{|l|c|c|c|c|c|c|c|}
\cline { 2 - 8 } \multicolumn{1}{c|}{} & Gesamt & & $\mathbf{1 9 6 9 - 1 9 7 8}$ & & $\mathbf{1 9 9 8 - 2 0 0 7}$ & & rel. Änderung \\
\cline { 2 - 9 } & Anzahl & in \% & Anzahl & in \% & Anzahl & in \% & in \% \\
\hline posttraumat. Pneumonie & 98 & 30,2 & 79 & 38 & 19 & 16,2 & $-\mathbf{5 7 , 2}$ \\
\hline posttraumat. Lungenembolie & 54 & 16,6 & 38 & 18,3 & 16 & 13,7 & $\mathbf{- 2 5 , 1}$ \\
\hline Sepsis & 52 & 16 & 28 & 13,4 & 24 & 20,5 & $\mathbf{+ 5 2 , 4}$ \\
\hline Aspiration & 34 & 10,5 & 4 & 1,9 & 30 & 25,6 & $\mathbf{+ 1 . 2 3 3 , 3}$ \\
\hline Sonstiges & 30 & 9,2 & 26 & 12,5 & 4 & 3,4 & $\mathbf{- 7 2 , 6}$ \\
\hline Fettembolie & 29 & 8,9 & 11 & 5,3 & 18 & 15,4 & $\mathbf{+ 1 9 0 , 9}$ \\
\hline Kombinationen & 18 & 5,5 & 13 & 6,2 & 5 & 4,3 & $\mathbf{- 3 1 , 6}$ \\
\hline Anaphylaxie/Allergie & 7 & 2,2 & 7 & 3,4 & 0 & 0 & $\mathbf{- 1 0 0}$ \\
\hline Luftembolie & 2 & 0,6 & 1 & 0,5 & 1 & 0,9 & $\mathbf{+ 7 7 , 8}$ \\
\hline Gesamt & 1 & 0,3 & 1 & 0,5 & 0 & 0 & $\mathbf{- 1 0 0}$ \\
\hline
\end{tabular}

Tab. 18: Mittelbare Todesursachen 1969-1978 und 1998-2007 


\subsubsection{Tötungsdelikte - Gewalteinwirkungen}

Die Verteilung der Arten der Gewalteinwirkung innerhalb der Kategorie „Tötungsdelikte“ ist Tabelle 19 zu entnehmen.

Die häufigste Form der Gewalteinwirkung war die scharfe Gewalt ( $n=171$, $\Delta=+56,8 \%$ ), gefolgt von der Einwirkung durch Schusswaffen ( $\mathrm{n}=166, \Delta=+65,5 \%)$. Am dritthäufigsten ließen sich Eintragungen der Gruppe „stumpfe Gewalt“ vorfinden $(n=143, \Delta=-40,3 \%)$.

\begin{tabular}{|c|c|c|c|c|c|c|c|}
\hline & Gesamt & & 1969-1978 & & 1998-2007 & & rel. Änderung \\
\hline & Anzahl & in $\%$ & Anzahl & in $\%$ & Anzahl & in \% & in $\%$ \\
\hline scharfe Gewalt & 171 & 28,1 & 94 & 23,5 & 77 & 36,8 & $+56,8$ \\
\hline Schuss & 166 & 27,3 & 89 & 22,2 & 77 & 36,8 & $+65,6$ \\
\hline stumpfe Gewalt & 143 & 23,5 & 109 & 27,2 & 34 & 16,3 & $-40,3$ \\
\hline \multicolumn{8}{|l|}{ Ersticken } \\
\hline Strangulation & 41 & 6,7 & 35 & 8,8 & 6 & 2,9 & $-67,2$ \\
\hline sonstiges Ersticken & 29 & 4,7 & 24 & 6 & 5 & 2,4 & $-60,1$ \\
\hline Ertrinken & 18 & 3 & 16 & 4 & 2 & 1 & $-76,1$ \\
\hline Gesamt & 88 & 14,4 & 75 & 18,8 & 13 & 6,3 & $-66,8$ \\
\hline halbscharfe Gewalt & 27 & 4,4 & 23 & 5,8 & 4 & 1,9 & $-66,7$ \\
\hline thermische Schädigung & 10 & 1,6 & 7 & 1,8 & 3 & 1,4 & -18 \\
\hline Intoxikation & 4 & 0,7 & 3 & 0,7 & 1 & 0,5 & $-36,2$ \\
\hline Gesamt & 609 & 100 & 400 & 100 & 209 & 100 & \\
\hline
\end{tabular}

Tab. 19: Art der Gewalteinwirkung bei Tötungsdelikten 


\subsubsection{Intoxikationen}

Die Tabellen 20 und 21 zeigen die Häufigkeit der Substanzen, die im Zusammenhang mit Intoxikationsfällen $(n=791)$ dokumentiert wurden. Diese Zahl übersteigt ebenfalls die unter Abschnitt 3.1.12 kategorisierten Intoxikationsfälle, da Überschneidungen mit übergeordneten Umständen (Tötung, Suizid, Unfall etc.) unvermeidbar waren. Auffallend ist ein starker Anstieg der Todesfälle, die mit „illegalen Drogen“ assoziiert sind. So hat der relative Anteil der Heroinfälle um $723,8 \%$ zugenommen $(n=101)$. Vergiftungen mit Methadon $(n=61)$, Kokain $(n=8)$ und Amphetaminen $(n=6)$ wurden nur im jüngeren Untersuchungszeitraum beobachtet. Rückgänge konnten bei Vergiftungen mit E605 ( $\mathrm{n}=17, \Delta=-100 \%)$, Barbituraten ( $\mathrm{n}=33, \Delta=-97,5 \%)$, Hypnotika $(\mathrm{n}=44, \Delta=-94,1 \%)$, Zyaniden $(\mathrm{n}=12$, $\Delta=-92,7 \%)$, Alkohol $(\mathrm{n}=81, \Delta=-41,7 \%)$ sowie Kohlenmonoxid $(\mathrm{n}=167, \Delta=-35,9 \%)$ festgestellt werden.

\begin{tabular}{|c|c|c|c|c|c|c|c|}
\hline & Gesamt & & 1969-1978 & & 1998-2007 & & rel. Änderung \\
\hline & Anzahl & in $\%$ & Anzahl & in $\%$ & Anzahl & in \% & in $\%$ \\
\hline Morphin/Heroin & 101 & 12,8 & 9 & 2,5 & 92 & 21 & $+723,8$ \\
\hline Mischintoxikationen & 171 & 21,6 & 63 & 17,9 & 108 & 24,7 & $+38,2$ \\
\hline Methadon & 61 & 7,7 & 0 & 0 & 61 & 13,8 & \\
\hline Kokain & 8 & 1 & 0 & 0 & 8 & 1,9 & \\
\hline Amphetamin & 6 & 0,8 & 0 & 0 & 6 & 1,4 & \\
\hline E 605 & 17 & 2,1 & 17 & 4,8 & 0 & 0 & -100 \\
\hline Barbiturate & 33 & 4,2 & 32 & 9,1 & 1 & 0,2 & $-97,5$ \\
\hline Hypnotika & 44 & 5,6 & 41 & 11,6 & 3 & 0,7 & $-94,1$ \\
\hline Zyanid & 12 & 1,5 & 11 & 3,1 & 1 & 0,2 & $-92,7$ \\
\hline Alkohol & 81 & 10,2 & 47 & 13,3 & 34 & 7,8 & $-41,7$ \\
\hline $\mathrm{CO}$ & 167 & 21,1 & 93 & 26,4 & 74 & 16,9 & $-35,9$ \\
\hline sonstige Intoxikation & 90 & 11,4 & 40 & 11,3 & 50 & 11,4 & $+0,7$ \\
\hline Gesamt & 791 & 100 & 353 & 100 & 438 & 100 & \\
\hline
\end{tabular}

Tab. 20: Intoxikation 1969-1978 und 1998-2007

Aufgrund der hohen Anzahl an Mischintoxikationen ( $n=171,21,6 \%$ ) wurden die Kombinationen der Substanzen mit Zuhilfenahme des Freitextes der Protokolle rekonstruiert (Tab. 21). Im ersten Untersuchungszeitraum (1969-1978) konnten die meisten Mischintoxikationen $(n=46,73,1 \%)$ als Alkoholmischintoxikationen 
klassifiziert werden, von denen 43 (68,3 \%) kombinierte Alkohol-MedikamentenIntoxikationen waren. In weiteren 3 Fällen lag eine Mischintoxikation aus Kohlenmonoxid und Alkohol vor. Mischintoxikationen mit Medikamenten wurden in 12 Fällen (19 \%) ermittelt. Bei Betrachtung des jüngeren Zeitraumes (1998-2007) dominieren andere Substanzen das Gesamtbild. Mischintoxikationen wurden überwiegend im Beisein der Betäubungsmittel Heroin, Kokain, Methadon sowie Amphetamin dokumentiert. Lediglich $10,2 \%$ der Fälle $(n=11)$ bzw. 8,3 \% $(n=9)$ waren als Mischintoxikation mit Alkohol und Medikamenten bzw. isolierte Medikamentenintoxikationen vertreten.

\begin{tabular}{|c|c|c|c|c|c|c|c|}
\hline & Gesamt & & 1969-1978 & & $1998-2007$ & & rel. Änderung \\
\hline & Anzahl & in \% & Anzahl & in $\%$ & Anzahl & in \% & in $\%$ \\
\hline Heroin + Medikamente & 20 & 11,7 & 1 & 1,6 & 19 & 17,6 & $+1.008,3$ \\
\hline Heroin + Alkohol & 10 & 5,8 & 0 & 0 & 10 & 9,3 & \\
\hline Heroin + Methadon & 4 & 2,3 & 0 & 0 & 4 & 3,7 & \\
\hline Heroin + Alkohol + Medikamente & 9 & 5,2 & 0 & 0 & 9 & 8,3 & \\
\hline Heroin + Methadon + Kokain & 1 & 0,6 & 0 & 0 & 1 & 0,9 & \\
\hline Methadon + Alkohol & 6 & 3,5 & 0 & 0 & 6 & 5,6 & \\
\hline Methadon + Medikamente & 19 & 11,1 & 0 & 0 & 19 & 17,6 & \\
\hline Methadon + Alkohol + Heroin + Medikamente & 1 & 0,6 & 0 & 0 & 1 & 0,9 & \\
\hline Methadon + Heroin + Alkohol + Medikamente & 1 & 0,6 & 0 & 0 & 1 & 0,9 & \\
\hline Methadon + Kokain & 1 & 0,6 & 0 & 0 & 1 & 0,9 & \\
\hline Methadon + Kokain + Medikamente & 2 & 1,2 & 0 & 0 & 2 & 1,9 & \\
\hline Alkohol + Kokain & 1 & 0,6 & 0 & 0 & 1 & 0,9 & \\
\hline Alkohol + Medikamente & 54 & 31,6 & 43 & 68,3 & 11 & 10,2 & $-85,1$ \\
\hline Alkohol + Heroin + Codein & 1 & 0,6 & 0 & 0 & 1 & 0,9 & \\
\hline Alkohol + Methadon + Medikamente & 2 & 1,2 & 0 & 0 & 2 & 1,9 & \\
\hline Heroin + Kokain & 2 & 1,2 & 0 & 0 & 2 & 1,9 & \\
\hline Alkohol $+\mathrm{THC}+$ Heroin & 1 & 0,6 & 0 & 0 & 1 & 0,9 & \\
\hline Medikamente & 21 & 12,3 & 12 & 19 & 9 & 8,3 & $-56,3$ \\
\hline sonstige & 10 & 5,8 & 4 & 6,3 & 6 & 5,6 & $-12,5$ \\
\hline Amphetamin + Medikamente & 1 & 0,6 & 0 & 0 & 1 & 0,9 & \\
\hline $\mathrm{CO}+$ Alkohol & 4 & 2,3 & 3 & 4,8 & 1 & 0,9 & $-80,6$ \\
\hline Gesamt & 171 & 100 & 63 & 100 & 108 & 100 & \\
\hline
\end{tabular}

Tab. 21: Mischintoxikationen 1969-1978 und 1998-2007 


\section{Diskussion}

\subsection{Diskussion der Methode}

Die im Rahmen dieser Arbeit ausgewerteten 5.190 Protokolle waren nicht standardisiert und wiesen eine zum Teil stark abweichende Struktur und Terminologie auf. Dies ist unter anderem auf die verschiedenen Dokumentatoren zurückzuführen. Die vom Obduzenten abhängigen Angaben (inter-observer variability) bzw. abweichende Interpretationen beeinflussen die Qualität der Daten maßgeblich. So gab es Fälle (z.B. Polytraumata bei Unfällen), bei denen von mehreren Verletzungen, die potenziell tödlich hätten verlaufen können, nur die zum Tode führende als todesursächlich dokumentiert wurde (z.B. Schädel-Hirn-Trauma). In anderen Fällen wurde als Todesursache lediglich „Polytrauma“ dokumentiert, sodass eine anatomische Zuordnung der zum Tode führenden Verletzung nicht erfolgen konnte. Ebenfalls ließen sich inhaltlich unvollständige, zum Teil auch widersprüchliche Angaben zu Todesumständen nicht vermeiden. So konnte beispielsweise nicht immer eindeutig zwischen einem Unfall und einem Suizid unterschieden werden (z.B. bei Stürzen).

\subsection{Allgemeine Betrachtung zum Obduktionsgut}

Insgesamt sind 5.190 Sektionen in die Studie eingegangen, von denen 2.501 (48,2 \%) in den Jahren von 1969 bis 1978 sowie 2.689 (51,8 \%) in den Jahren von 1998 bis 2007 ausgewertet wurden. Dieser Anstieg der Sektionshäufigkeit ist auf verschiedene Gründe zurückzuführen. Innerhalb des 40-jährigen Zeitraumes kam es zu erheblichen Veränderungen innerhalb des rechtsmedizinischen Versorgungsgebietes, die es erschwerten, die Sektionszahl in Relation zu dem Einzugsgebiet zu setzen. Die früher noch strikt auf Niedersachsen beschränkte Zuständigkeit hatte sich nach Jahrzehnten auf Nordhessen ausgeweitet. Durch die Gründung der $\mathrm{MHH}$ (Medizinische Hochschule Hannover) im Jahre 1961 und den dortigen Lehrstuhl für Rechtsmedizin (1977) sowie deren Zweigstelle in Oldenburg wurde der rechtsmedizinische Zuständigkeitsbereich des hiesigen Instituts für Nordniedersachsen weiter eingeschränkt. Es sei noch erwähnt, dass der aktuelle 
Zuständigkeitsbereich seit dem Jahr 2008 verkleinert wurde, da die Region Nordhessen nur noch bedingt durch die Rechtsmedizin Göttingen versorgt wird. Die Sektionszahl des hiesigen Instituts liegt seit 2008 bei durchschnittlich 100-150 pro Jahr. Durch die sich verändernden Versorgungsbereiche wäre eine genaue Bestimmung der Sektionsfrequenz nur mit sehr hohem Aufwand möglich. Demnach sollten Veränderungen im Einzugsbereich als möglicher Faktor für Verzerrungen der Ergebnisse gesehen werden.

In der vorliegenden Arbeit ging der leichte Anstieg gerichtlicher Sektionen mit einem erheblichen Rückgang bei den Verwaltungssektionen einher. Der Rückgang der klinischen bzw. Verwaltungssektion wurde ebenfalls an anderen rechtsmedizinischen Instituten beobachtet. So konnte ein Rückgang von 29 \% (1978-1980) auf 0,2 \% (1993-1995) am rechtsmedizinischen Institut in Frankfurt am Main festgestellt werden (Knobloch 1996, Herden 2008). Ebenfalls wurde ein Rückgang von 37 \% (19781982) auf 5,5 \% (1994-1998) in Hannover (Schulz 2007) nachgewiesen. Bundesweit konnte ein Rückgang der Sektionsfrequenz von 4,2 \% (1994) auf 3,1 \% (1999) an allen pathologischen Prosekturen ermittelt werden, während der Anteil richterlich angeordneter Sektionen auf dem niedrigen Niveau von ca. $2 \%$ stagnierte (Brinkmann et al. 2002). Die Gesamtsektionsfrequenz lag demnach im Jahr 1999 bei 5,3 \%. Zum Vergleich: Im Jahre 1992 betrug die Obduktionsfrequenz (klinischpathologisch und rechtsmedizinisch) in Schweden $22 \%$, in Finnland $31,1 \%$, in Dänemark $16 \%$, in der Schweiz (2002) $20 \%$ und in Österreich (1999) 30-35\% (Bundesärztekammer 2005, Brinkmann et al. 2002). Als mit ursächlich für den Rückgang klinischer Sektionen wird die uneinheitliche Rechtslage diskutiert, die durch die jeweiligen Landesgesetze geregelt wird (Madea et al. 2006b, Dettmeyer und Madea 2002). Die früher angewandte Widerspruchslösung, in der Angehörige innerhalb einer festgesetzten Frist einer Sektion widersprechen konnten, wurde in vielen Bundesländern durch die Zustimmungs- bzw. Einwilligungslösung ersetzt. Diese erfordert ein aktives Einholen der Einwilligung bei den Angehörigen, wenn der Verstorbene nicht schon zu Lebzeiten einer Sektion zugestimmt hatte. Folglich fiel der Anteil der klinischen Sektionen, wie auch von Georgii und Meliss (1992) unabhängig bestätigt wurde. Weitere Gründe für den Rückgang liegen in „mangelnder Erfahrung klinisch tätiger Ärzte, Angehörigengespräche zu führen, Angst vor Aufdeckung eigener Fehler, fehlendem Bewusstsein über den 
Erkenntniswert einer Obduktion, Kostendenken und Sparzwang, unklarer Rechtslage und geringen Attraktivität von Publikationen auf der Basis von Obduktionsergebnissen“ (Bundesärztekammer 2005, S. 8-9). Das fehlende Engagement der klinisch tätigen Ärzte, adäquate Angehörigengespräche zu führen, wurde mehrfach kritisiert (Sperhake und Püschel 2003, Brinkmann et al. 2002). Sperhake und Püschel (2003) empfahlen zur Vertrauensbildung und höheren Akzeptanz ein durch Pathologen geführtes Angehörigengespräch.

Trotz der Verbesserung diagnostischer Methoden (Bildgebung, Laborchemie etc.) gibt es nach wie vor Diskrepanzen zwischen klinischer und autoptischer Diagnose (Bauer et al. 1991, Modelmog 1993). Modelmog (1993) wies bei fast 100-prozentiger Sektionsquote („Görlitzer Studie“) eine fehlende Übereinstimmung von 47,2 \% zwischen klinischer und autoptischer Diagnose nach. In etwa $25 \%$ dieser Fälle hätte die autoptische Diagnose eine klinische Konsequenz gehabt („Fehldiagnose“). Weiterhin konnte nachgewiesen werden, dass die Häufigkeit der Abweichungen bzw. Übereinstimmungen stark mit dem Sterbeort korrelieren. Gerade bei nicht im Krankenhaus Verstorbenen lässt sich die genaue Todesursache nicht feststellen. Daher ist es schwer nachvollziehbar, dass klinische Sektionen in diesen Fällen äußerst selten durchgeführt werden. Zur Lösung des Problems wurde eine gesetzliche Regelung für die Durchführung von Verwaltungssektionen bei medizinisch unklaren Todesfällen gefordert (Dettmeyer und Madea 2002, Madea et al. 2006a).

Die gerichtliche Sektion ist gemäß $\S \S 87$ ff. StPO geregelt. Sie dient zur Klärung der Frage nach einem nicht natürlichen Tod, einem Fremdverschulden und der Sicherung von Beweisen für einen möglichen Strafprozess (Bundesärztekammer 2005, s. §§ 87 ff. StPO). Die tatsächliche Anordnung unterliegt jedoch einem großen Ermessensspielraum der Staatsanwaltschaft (Madea et al. 2006b). Schätzungsweise wird nur jeder 10. bis 20. unklare Todesfall gerichtlich obduziert (Madea et al. 2006a). In lokalen Untersuchungen zu Angaben aus den Todesbescheinigungen $(n=2.170 ; n=5.603 ; n=3.670)$ wurde die Klassifizierung der natürlichen Todesart (vor möglichen Obduktionen) in 91,3 \% (Nick 2006), 93,7 \% (Berster 2006) und 95 \% (Koch 2004) der Fälle ermittelt. Das Statistische Bundesamt gab für das Jahr 2007 den Anteil nicht natürlicher Todesfälle mit ca. 4-6,5 \% an. Die „Görlitzer Studie“ ergab jedoch in 8,5-9 \% aller Fälle eine nicht natürliche Todesart (Modelmog 1993) und lässt zu Recht an der Validität der Todesursachenstatistik zweifeln. Zahlreiche 
internationale Untersuchungen bestätigten ebenfalls Diskrepanzen zwischen Leichenschaudiagnosen und Autopsiebefunden (Dash et al. 2014, Ravakhah 2006, Coradazzi et al. 2003, Ashworth 1991). Brinkmann et al. (1997b) schätzten in einer bundesweiten multizentrischen Studie die Zahl unerkannter nicht natürlicher Todesfälle auf 11.000-22.000 pro Jahr, darunter 1.200-2.400 Tötungsdelikte. Ursächlich seien unter anderem die Schwächen des deutschen Leichenschausystems und die nicht ausreichende Ausbildung der Ärzte im Leichenschauwesen. Madea (2007) führte eine Reihe von Problemen bei der ärztlichen Leichenschau auf (s. Abb. 19). Den niedergelassenen Ärzten, die häufig gesamte Familien betreuen, würde es im Falle einer häuslichen Leichenschau unter Anwesenheit Angehöriger schwer fallen, die Aufgabe des „sachlich-neutralen Gutachters“ professionell auszuführen. Unter dieser emotionalen Situation sei es schwierig, eine nicht natürliche bzw. ungeklärte Ursache zu attestieren, da man dem Vertrauensverhältnis keinen Schaden zufügen möchte. Weiterhin wurde von Beeinflussungsversuchen durch polizeiliche Mitarbeiter berichtet. Diese drängen auf die Attestierung eines natürlichen Todes oder fordern unter Umständen eine neue Bescheinigung, sofern eine ungeklärte oder nicht natürliche Ursache durch andere Kollegen attestiert wurde (Rothschild 2009, Vennemann et al. 2001, Brinkmann et al. 1997b, Thomsen und Schewe 1994). Das Verhalten der polizeilichen Ermittler lässt sich durch den „Versuch der Vermeidung als unnötig angesehene Ermittlungsarbeit“ erklären (Rothschild 2009).

Ein weiteres tabuisiertes Problem ist die geringe Honorierung der ärztlichen Leichenschau mit ca. 15-34 Euro (Hefer und Wenning 2006). In einer Befragung zufällig ausgesuchter leichenschauender Ärzte (Vennemann et al. 2001) hatten lediglich $25 \%$ angegeben, die Leichenschau an einem vollständig entkleideten Leichnam durchzuführen. $20 \%$ der niedergelassenen Ärzte wurden durch Angehörige beeinflusst, zwei Drittel berichteten über nachteilige Folgen nach Angabe einer nicht natürlichen bzw. unklaren Todesart. Bei der Einschätzung typischer Fallkonstellationen gab lediglich ein Fünftel aller Ärzte bezüglich eines „Mors in tabula" die Todesart als ungeklärt an und drei Viertel aller Internisten würden eine Pneumonie 14 Tage nach Schenkelhalsfraktur mit ständiger Bettlägerigkeit als natürlich klassifizieren. Unter solchen Umständen sei das Übersehen nicht natürlicher Anhaltspunkte praktisch vorprogrammiert (Vennemann et al. 2001). Viele 
Ärzte sehen sich mit dem Aufgabenkanon der Leichenschau überfordert, die neben Feststellung der Todesart dem Arzt zusätzliche "hoheitliche" Aufgaben (Verbrechensverfolgung, Todesursachenstatistik für gesundheitspolitische Entscheidungen und die Seuchenbekämpfung) abverlangt (Hefer und Wenning 2006). Daher fordern die Autoren eine Intensivierung der Ausbildung leichenschauender Ärzte bzw. die Durchführung der Leichenschau durch spezialisierte Leichenschauärzte (Scheib 2002, Rothschild 2009, Vennemann et al. 2001, Püschel 2009).

- Tabelle 2.2. Ursachenkomplexe für die Misere der ärztlichen Leichenschau

Strukturelle Ursachen

- Keine bundeseinheitliche Regelung

- Unzureichend ausgestaltete, zu wenig ausdifferenzierte Todesbescheinigungen

- Mangelnde Verbalisierung der Aufgabenkomplexe im Sinne einer Checkliste

- Für bestimmte Fallgruppierungen objektive Überforderung des Leichenschauers ohne flexible Lösungsmöglichkeiten (2. fachärztliche Leichenschau oder 2. Leichenschau durch Hausarzt)

- Fehlende Definition des natürlichen und nichtnatürlichen Todes mit einseitiger Ausrichtung auf »Fremdverschulden«

- Verquickung ärztlicher mit kriminalistischen Aufgaben

Ursachen auf Seiten des Arztes

- Leichenschau wird als Aufgabe jenseits des eigentlichen ärztlichen Heilauftrags gesehen.

- Fehlende Vorbildung in der Handhabung von Problemfällen (spurenarmer gewaltsamer Tod, fortgeschrittene Leichenerscheinungen, unerwartete Todesfälle, Leichnam nicht identifiziert).

- Neigung als Leichenschauer, sich und den Angehörigen »Ärger vom Hals zu halten « mit leichtfertiger Attestierung eines natürlichen Todes (Motiv: Verlust von Patienten oder einer Anstellung als Heimarzt, Gerede über den Arzt, der nicht einmal in der Lage sei, einen natürlichen Tod festzustellen).

- „Obrigkeitshörigkeit« gegenüber der Polizei mit oftmals allzu willfähriger Gewährung von Auskünften oder Ausfüllung weiterer Leichenschauscheine.

- Resignation verantwortungsbewusst handelnder Ärzte im Hinblick auf die kriminalpolizeiliche Handhabung nicht geklärter Todesfälle.

- Unzureichende Honorierung im Hinblick auf eine der verantwortungsvollsten ärztlichen Diagnosen.

Ursachen auf Seiten der Ermittlungsbehörden

- Pressionen von Seiten der Polizei auf Attestierung eines natürlichen Todes

- Statt Ermittlungen zur Aufklärung nicht geklärter Todesfälle Beschaffung weiterer Leichenschauscheine (entgegen der Verpflichtung zur Meldung an die StA nach $§ 159$ StPO) oder vollständiger »Ermittlungsquietismus«

Situative Ursachen

- Pressionen von Seiten der Angehörigen oder der Heimleitung auf Attestierung eines natürlichen Todes

- Durchführung der Leichenschau erschwert bis objektiv unmöglich (Leichen im Freien, in der Öffentlichkeit, fortgeschrittene Leichenerscheinungen, übergroßes Körpergewicht, keine Gehilfen zur Verfügung)

Abb. 19: Madea 2007, S.10. Mit freundlicher Genehmigung des Springer Verlags

Von den wenigen ungeklärten bzw. nicht natürlich gemeldeten Fällen (bundesweit ca. $8 \%$ ) wird nur jeder vierte Fall einer rechtsmedizinischen Untersuchung zugeführt (Brinkmann et al. 2002). In vergleichbaren Ländern wird die Attestierung eines nicht natürlichen bzw. ungeklärten Todes in 15-20 \% der Fälle vorgenommen (Brinkmann und Püschel 1991). Als Konsequenz einer niedrigen Sektionsrate steigt die Exhumierungsrate (Banaschak et al. 1998), welches als strukturelle Schwäche im deutschen Leichenschau- und Todesermittlungssystem interpretiert werden kann. In 
der Studie konnte eine umgekehrte Proportionalität zwischen Sektionshäufigkeit und Exhumierungsrate („Reziprozitätsprinzip“) ermittelt werden.

\subsection{Geschlechterverteilung}

In beiden Zeiträumen war der Anteil männlicher Verstorbener etwa doppelt so hoch wie der weiblicher Verstorbener. Dieses Verhältnis wurde bereits in anderen Sektionsstudien beobachtet (Moschkau et al. 2011, Oehme 2012, Opitz 2012, Schulz 2007, Larsen und Lynnerup 2011). Geschlechtsspezifische Attribute wie beispielsweise Gewaltbereitschaft, Fehleinschätzung eigener Fähigkeiten, Rivalität und Risikobereitschaft lassen Männer häufiger eines nicht natürlichen Todes versterben (Bennet et al. 2005, Baxendale et al. 2012). Doch auch unter den natürlichen Todesarten sind Männer stärker vertreten. Ein möglicher Grund ist die zunächst häufigere Attestierung eines ungeklärten Todes bei plötzlich Verstorbenen, der sich im Laufe der rechtsmedizinischen Untersuchung als natürliche Todesart herausstellt.

\subsection{Sterbealter}

In der vorliegenden Arbeit stieg der statistische Mittelwert des Sterbealters von 37,8 (1969-1978) auf 46,8 Jahre an. Die deutlichen Veränderungen in der Altersverteilung stimmen mit der demographischen Entwicklung sowie dem Rückgang der Säuglingsund Kindersterblichkeit (Statistisches Bundesamt - Eheschließung, Geborene, Gestorbene) überein. Eine annähernde Übereinstimmung des durchschnittlichen Sterbealters (im jüngeren Zeitraum) konnte auch in weiteren rechtsmedizinischen Studien ermittelt werden. Schulz ermittelte ein Durchschnittsalter von 45,7 Jahren und Herden (2008) gab 52,9 Jahre an. Der Rückgang der Säuglings- und Kindersterblichkeit in Industrienationen wurde von Razum und Breckenkamp (2007) seit Beginn des 20. Jahrhunderts mit wachsendem Wohlstand, sozialen und hygienischen Fortschritten sowie Verbesserungen der pädiatrischen Versorgung (Impfungen und antibiotischer Therapie) begründet. Seit 1970 wurde insbesondere die Frühgeborenenüberlebensrate durch „leistungsfähige und kostenintensive Geburts- und Perinatalmedizin“ verbessert (Razum und Breckenkamp 2007, S. 2953). Weiterhin lässt sich der Anstieg des mittleren Sterbealters durch den 
höheren Anteil der Fälle erklären, die aufgrund des Verdachts eines Behandlungsfehlers obduziert wurden. Die Verstorbenen hatten in den Jahren 1998-2007 ein deutlich höheres mittleres Sterbealter (60,7 Jahre).

\subsection{Todesarten}

Beim Vergleich der Verteilung der Todesarten dieser Studie (nicht natürlich: 59,4 \%, natürlich: 33,4 \% und ungeklärt: 7,2 \%) mit den Ergebnissen anderer Autoren zeigt sich ein weitgehend übereinstimmendes Bild:

Schulz (2007) gab folgende Verteilung an: 52,1 \% nicht natürliche, 34,4 \% natürliche und 12,3 \% ungeklärte Todesfälle. Bei Jäger (2008) war der Anteil der nicht natürlichen Todesarten 42,4 \%, der Anteil der natürlichen Fälle 53,3 \%, und 4,3 \% der Fälle wurden als ungeklärt klassifiziert. Opitz (2012) ermittelte einen Anteil nicht natürlicher Fälle von 69,3 \% und einen Anteil natürlicher Fälle von 25,6 \%. 4,8 \% der Fälle wurden als ungeklärt angegeben. In der Auswertung von Kausche (1998) lag der Anteil natürlicher Todesfälle bei 30,5 \%, der Anteil der nicht natürlichen bei $68 \%$ und in 1,2 \% der Fälle war die Ursache ungeklärt. Die Diskrepanz des Anteils ungeklärter Fälle ist groß (1,2-12,3 \%). Die in dieser Arbeit ermittelten Werte liegen in der oberen Hälfte, jedoch bedarf der relative Anstieg von 29,4 \% bei dieser Todesart innerhalb von 40 Jahren einer Erklärung. Grundsätzlich hat sich die Todesursachenfeststellung mithilfe der Entwicklung diagnostischer Methoden (Alkohologie, Toxikologie, Histologie, humangenetische Untersuchung, molekularpathologische Untersuchungen) über die Jahrzehnte verbessert. Madea et al. (2006b) konstatierten, dass unter Ausschöpfung weiterführender Untersuchungen ca. 95-97\% aller Todesfälle geklärt werden können. Sogar Erkrankungen, für die kein morphologisches Korrelat erkennbar ist (z.B. Herzrhythmusstörungen), können mit Hilfe molekular-pathologischer Untersuchungen diagnostiziert werden. Der Grund für den dennoch hohen Anteil ungeklärter Fälle ist die Haltung der Staatsanwaltschaft gegenüber einer „medizinischen“ Aufklärung. Sollte sich während einer Sektion kein Anhalt auf ein Fremdverschulden ergeben, werden in der Regel keine weiterführenden Untersuchungen in Auftrag gegeben (Madea et al. 2006b). 


\subsection{Natürliche Todesart}

Im natürlichen Todesursachenspektrum dominieren Erkrankungen des HerzKreislauf-Systems nach wie vor das Gesamtbild. Vom Statistischen Bundesamt wurde für das Jahr 2012 ein Anteil von 40,2 \% für Herz-Kreislauf-Erkrankungen innerhalb aller Todesarten (natürlich und nicht natürlich) ermittelt (Statistisches Bundesamt 2013). Im vorliegenden Obduktionsgut wurde innerhalb der Gruppe "natürliche Todesart" ein Anteil von $49 \%$ ermittelt. Bei der Altersgruppe der Kinder (bis einschließlich 5. Lebensjahr) wurde in den Jahren 1969-1978 in $89 \%$ (s. 3.2.) der Fälle eine respiratorische Erkrankung als todesursächlich bzw. mit ursächlich angegeben. Im jüngeren Zeitraum (1998-2007) lag dieser Anteil bei lediglich 6,6 \%. Ursächlich für den Rückgang der Kindersterblichkeit (bis 5. Lebensjahr) in Industrienationen zwischen 1970 und 2004 sei eine bessere Verfügbarkeit von Antibiotika und Impfungen, die zu einem Rückgang der Kindersterblichkeitsrate von 27/1000 auf 6/1000 führte (Razum und Breckenkamp 2007).

\subsection{Nicht natürliche Todesart}

\subsubsection{Intoxikation}

Bei Betrachtung der Todesumstände nicht natürlich klassifizierter Todesfälle ist ein starker Anstieg erfasster Drogentodesfälle erkennbar. In dem hier untersuchten Kollektiv ist die Anzahl der Todesfälle mit Rauschgiftbeteiligung um mehr als das 17Fache angestiegen. Die Anzahl Drogentoter in Westdeutschland wurde im Jahr 1973 mit 106 beziffert (Bundeskriminalamt 2010). Der Wert stieg diskontinuierlich an und lag im Jahr 2007 bei 1396 Rauschgifttodesfällen (s. Abb. 20). Seit dem Jahr 2000 $(n=2.030)$ ist eine rückläufige Tendenz zu beobachten (2013; $n=1.002)$. Durch die stärkere Verbreitung des Heroins in den 80er Jahren und dem Beginn der Methadonsubstitutionstherapie Anfang der 90er Jahre, stehen diese Substanzen im Vordergrund (Bundeskriminalamt 2010). Die rückläufige Tendenz von Herointoten bei gleichzeitigem Anstieg Methadon-assoziierter Todesfälle ist ebenfalls in einer dänischen Studie (Simonsen et al. 2011) zu beobachten. Riemann (2003) gab mehrere Gründe für den Rückgang Herointoter im Frankfurter Raum an: Es seien im 
Rahmen der Drogenhilfe Methadonsubstitutionsbehandlungen, Therapieplätze, betreute Wohngemeinschaften und Konsumräume mit Fachpersonal und sterilem Zubehör eingeführt worden, um die Zahl der Drogentoten und drogenassoziierten Erkrankungen (Hepatitiden, HIV etc.) zu senken.

Heinemann et al. (1998) berichteten ebenfalls über den starken Anstieg der Methadon-assoziierten Todesfälle der Jahre 1990-1996, stellten jedoch klar, dass bei Substituierten die Letalität signifikant niedriger ist als bei Nicht-Substituierten. Intoxikationsfälle mit E 605, Barbituraten, Hypnotika und Zyankali sind vollständig bzw. sehr stark zurückgegangen. Für den Rückgang spielen verschärfte Abgabebedingungen bzw. seltener therapeutischer Einsatz eine Rolle.

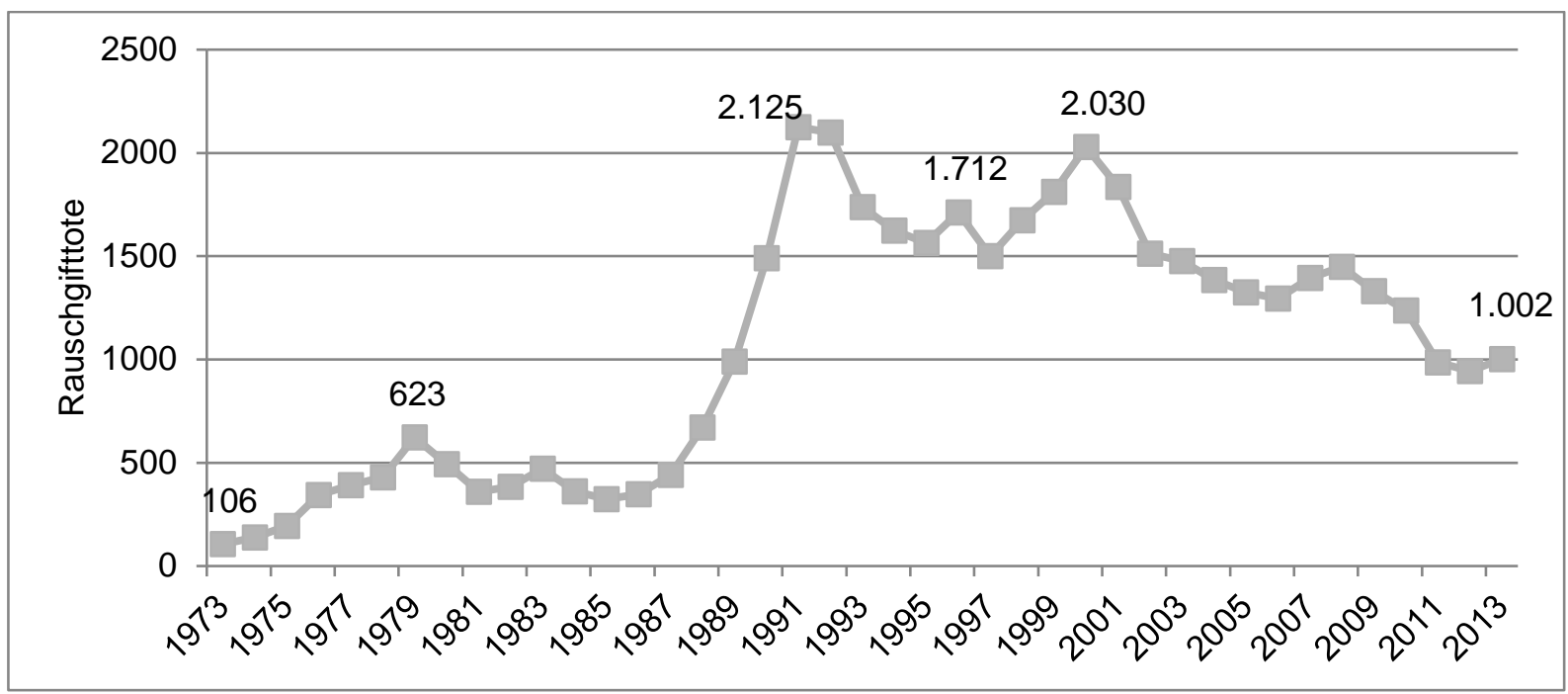

Abb. 20: Zahl der Rauschgifttoten in der Bundesrepublik Deutschland (1973-1990 alte Bundesländer, 1991 alte Länder mit Gesamt-Berlin, ab 1992 gesamtes Bundesgebiet). Quelle: Bundeskriminalamt 2002 und 2014

\subsubsection{Unfälle (Arbeitsunfälle/Verkehrsunfälle)}

Opfer von Arbeits- bzw. Verkehrsunfällen können einer rechtsmedizinischen Untersuchung unterzogen werden, um die Kausalität zwischen Unfallereignis und Todeseintritt festzustellen. Durch Untersuchungen kann der Unfallhergang analysiert werden, um beispielsweise ein Fremdverschulden nachzuweisen. Daher können sich für Angehörige von Unfalltoten Ersatzansprüche, beispielsweise in Form einer Hinterbliebenenrente, ergeben (Schieche et al. 2000). 
In dieser Arbeit kam es zu Überschneidungen zwischen der Kategorisierung von Arbeits- und Verkehrsunfällen, sofern eine Dokumentation über einen Arbeits- bzw. Wegeunfall vorlag. Daher gab es die Möglichkeit, die Form der Verkehrsbeteiligung zusätzlich zu erfassen. In unserer Auswertung nahm die Anzahl kategorisierter Verkehrsunfälle von 339 auf 212 und die der Arbeitsunfälle von 66 auf 28 ab. Die Zahl der tödlichen Arbeitsunfälle war bundesweit von 4.262 (1970, alte Bundesländer) auf $812 \mathrm{im} \mathrm{Jahr} 2007$ gesunken (BAuA - Zeitreihen 2009). Ebenso ist die Zahl der Verkehrstoten von 21.332 auf 4.949 gesunken, obwohl in diesem Zeitraum gleichzeitig der Fahrzeugbestand von 20,8 Mio. auf 50,9 Mio. angestiegen ist (Statistisches Bundesamt 2011). Für den Rückgang spielen Verbesserungen der Sicherheitssysteme, strengere Vorschriften in Bezug auf die passive Sicherheit (Helm- und Gurtpflicht) im Straßenverkehr und die Senkung der Höchstgrenze des Blutalkoholkonzentrationswertes eine wichtige Rolle. Weitere Gründe sind Verbesserungen in der Verkehrserziehung, Fortschritte in der medizinischen Erstversorgung, vermehrte Verkehrskontrollen und Einrichtung von Fußgängerzonen und Radwegen, die geschützte (z.B. Pkw-Insassen) von ungeschützten (z.B. Fußgänger und Fahrradfahrer) trennen (Statistisches Bundesamt 2011). Diese führten auch zu einer simultanen Abnahme der Arbeitsunfälle, da in den 70er Jahren etwa $45 \%$ (alte Bundesländer) als Wegeunfälle klassifiziert wurden (BAuA Zeitreihen - Unfallgeschehen 2009).

\subsubsection{Suizide}

Der Anteil der Suizide im untersuchten Sektionsgut war zunehmend und stellt einen Gesamtanteil von 9,2 \% der nicht natürlichen Todesfälle dar. Jäger (2008) und Schulz (2007) ermittelten einen Anteil von 38,1 \% und 18,8 \%. Die Daten des Statistischen Bundesamtes zeigen einen Rückgang der bundesweit erfassten Sterbefälle durch vorsätzliche Selbstbeschädigung von 18.451 (1980) auf 9.402 (2007). In der Altersgruppe der 20- bis 44-Jährigen (Europäische Union) liegt der Anteil der Suizide bei etwa einem Drittel und stellt die zweithäufigste Todesursache dar (Niederländer 2006). Die bundesweite Tendenz stimmt nicht mit der in dieser Untersuchung vorliegenden Entwicklung überein. Hierfür gibt es mehrere Ursachen: Zum einen wird nicht jeder Suizid zwangsläufig obduziert, zum anderen gibt es eine 
Reihe von Grenzfällen (borderline cases), die eine klare Trennung zwischen Suiziden und Unfällen erschweren (Huusko und Hirvonen 1988). Hierdurch entstehe ein nicht unerhebliches Dunkelfeld. Schmidtke et al. (2009) gaben ebenfalls an, dass man gerade bei älteren Personen und Schwerstkranken von einer hohen Dunkelziffer ausgehen müsse, da ähnlich wie bei den Tötungsdelikten, ebenfalls „spurenarme“ Möglichkeiten der Tötung (z.B. Intoxikation) häufig praktiziert werden, die dem leichenschauenden Arzt nicht immer auffallen. Vennemann et al. (2006) gehen von einer Unterschätzung der amtlichen Suizidrate aus, da durch unterschiedliche Erfassung bei den Gesundheitsämtern systematische Fehler in der Gesamtstatistik auftreten. Bezüglich der Geschlechterverteilung (männlich: 73,2 \%, weiblich: 26,8 \%) finden sich Übereinstimmungen mit denen anderer Institute. Schulz (2007) ermittelte einen Anteil männlicher Suizidenten von $74 \%$, Jäger (2008) von 68,2 \%, Alamuti (1997) von 66\%, Kausche (1998) von $63 \%$ und Riemann (2003) von 70,3\%.

\subsubsection{Tötungsdelikte}

Die Zahl der Fälle die der Kategorie Tötungsdelikte zugeordnet wurde, ging von 339 (1969-1978) auf 163 (1998-2007) herunter. Schulz (2007) gab einen Rückgang von 208 (1978-1982) auf 148 (1994-1998) Fälle an. Ebenfalls rückläufig war die Zahl der erfassten Tötungsdelikte der Jahre 1981-1983 $(n=191)$ und 1999-2001 $(n=142)$ im rechtsmedizinischen Institut Frankfurt am Main (Alamuti 1997, Herden 2008).

In der vorliegenden Arbeit war die häufigste Art der Gewalteinwirkung in der Kategorie „Tötungsdelikte" die scharfe Gewalt, gefolgt von der Schusseinwirkung und der stumpfen Gewalt. Riemann (2003) und Jäger (2008) kamen zu einer übereinstimmenden Verteilung. Oehme (2012) gab als häufigste Gewalteinwirkung die scharfe Gewalt, gefolgt von der stumpfen Gewalt und der Strangulation an. Schulz (2007) und Fischer et al. (1994) ermittelten als führende Gewalteinwirkung die stumpfe Gewalt, gefolgt von der scharfen Gewalt und dem Ersticken an dritter Stelle. Internationale Studien zeigen deutlich höhere Anteile von Tötungsdelikten, die durch Schusswaffen begangen wurden. Nordrum et al. (1998) ermittelten für die Stadt Tromsø (Norwegen) den Schusswaffengebrauch als zweithäufigste Gewalteinwirkungsart im Zusammenhang mit Tötungsdelikten. Fernandez und La Harpe (1996) konnten in ihrer Untersuchung eine annähernd gleiche Häufigkeit von scharfer 
Gewalt und Schusseinwirkung bei Tötungsdelikten im Kanton Genf feststellen. In der Studie von Price et al. (1997) wurde nachgewiesen, dass $70 \%$ aller Tötungsdelikte in den USA auf Schusswaffengebrauch zurückzuführen sind. Eine Korrelation zwischen der Häufigkeit von Tötungsdelikten durch Schusswaffengebrauch und dem Besitz von Schusswaffen wurde durch Killias (1993) bestätigt.

Die Zahl der erfassten vollendeten Tötungsdelikte (alte Bundesländer) lag 1970 bei 779 (Bundeskriminalamt 1970). Diese ging nach der Wiedervereinigung auf 692 (2007) leicht herunter (Bundeskriminalamt 2007). Die tatsächliche Zahl von Tötungsdelikten ist Gegenstand vieler Untersuchungen („Dunkelfeldforschung“) und wird von Rechtsmedizinern und Kriminologen deutlich höher geschätzt als die Angaben der polizeilichen Kriminalstatistik. Die Erforschung des Dunkelfeldes gestaltet sich als sehr schwierig: Da eine Opferbefragung naturgemäß nicht stattfinden kann, müssen andere Methoden mit eingeschränkter Aussagekraft angewandt werden. Harbort (2001) beschrieb in seinem Werk, dass Serienmörder immer wieder Morde gestanden, die ihnen nicht vorgeworfen wurden. Den Opfern wurde eine natürliche bzw. ungeklärte Todesursache attestiert (oder sie waren als vermisst gemeldet) und eine umfangreiche Aufklärung blieb aus. Der Versuch der Ermittlungsbehörden, nachträglich doch noch Beweise und Befunde zu sichern, spiegelt sich in einer hohen Zahl von Exhumierungen wider - Deutschland besitzt eine der höchsten Exhumierungsraten weltweit (Brinkmann et al. 2002). Diese können wegen postmortalen Fäulnisveränderungen nicht immer die nötigen Erkenntnisse liefern. Die „Projektstudie über das Mobilitätsverhalten von Serien- und Intensivtätern“ führte eine anonyme Befragung an 302 Strafgefangenen durch. $26 \%$ $(\mathrm{n}=80)$ nahmen an der Befragung teil. Die Zahl der unerkannten Delikte war acht Mal häufiger als die der ermittelten. $77 \%$ der Befragten beantworteten Fragen zu der Rubrik Kapitalverbrechen. 9 \% dieser Befragten haben dabei ein Kapitalverbrechen gestanden, von denen Ermittlungsbehörden keine Kenntnis hatten. Eine weitere Methodik der Dunkelfeldforschung besteht darin, Zufallsentdeckungen hochzurechnen, wie zum Beispiel in der multizentrischen Studie von Brinkmann et al. (1997a und 1997b). Weiterhin kann man Vergleiche zwischen Regionen mit einem mutmaßlich hohen Dunkelfeld (BRD) und Regionen, in denen bestimmte Todesfälle nahezu lückenlos aufgeklärt wurden (DDR), ziehen. Vock et al. (1999) untersuchten tödliche Kindesmisshandlungen im Zeitraum 1985-1990, die in der DDR nahezu 
doppelt so häufig ermittelt wurden. Aufgrund einer Sektionsanordnung für alle kindlichen Leichenfälle einschließlich des 16. Lebensjahres, sei hier ein niedrigeres Dunkelfeld zu erwarten. In einer weiteren Studie (Du Chesne et al. 1996) wurde im gerichtlich angeordneten Sektionsgut in $3 \%$ aller Fälle eine Kindstötung nachgewiesen. Bei der Vergleichsgruppe, in der die Sektionen aufgrund von wissenschaftlichem Interesse („Kindstodsforschungsprogramm“) erfolgte, wurde in 2,8\% der Fälle ein Tötungsdelikt nachgewiesen. Die Ergebnisse lassen „Ineffektivität der staatsanwaltschaftlichen Auswahlkriterien bei Kindertodesfällen vermuten“ (Du Chesne et al. 1996, S.26). Aus diesen Studien kann man schlussfolgern, dass eine höhere Sektionsfrequenz zur Aufdeckung von nicht natürlichen Todesfällen bzw. Tötungsdelikten führt.

Scheib (2002) arbeitete in seiner Dissertation „Die Dunkelziffer bei Tötungsdelikten aus kriminologischer und rechtsmedizinischer Sicht" mögliche Ursachen des großen Dunkelfeldes auf, die seiner Ansicht nach durch systematische Mängel in der Todesermittlungskette zu erklären sind, beginnend bei der Praxis der ärztlichen Leichenschau.

Die Autoren Sellin und Weber (2001) sowie Rückert (2002) führten Recherchen bei Ermittlern, Rechtsmedizinern, Richtern und Staatsanwälten durch und schrieben in ihren Werken in exemplarischen Fallbeispielen über Mängel im deutschen Ermittlungssystem und das Desinteresse der Politiker, Reformen durchzuführen. Durch eine Reformierung des bestehenden Leichenschau- und Ermittlungssystems würden sich die Behauptungen der Kriminologen und Rechtsmediziner bewahrheiten und ein Anstieg der Mord- bzw. Tötungsdelikte wäre die Folge.

\subsubsection{Behandlungsfehler}

In der vorliegenden Arbeit führten die vermehrten Verdachtsmomente in Bezug auf Behandlungsfehler zu einem Rückgang bestätigter Fehler (-84 \%) und zu einem starken Anstieg der Fälle, in denen ein Behandlungsfehler ausgeschlossen wurde (+71,1 \%). Über deutliche Zunahmen von Sektionen aufgrund eines Behandlungsfehlervorwurfs wurde auch in anderen Studien berichtet (Casali et al. 2014, Dettmeyer und Madea 1999, Preuß et al. 2006, Schulz 2007). Trotz dieser Ergebnisse sollte der hohe Stellenwert der Sektion in diesen Fällen nicht außer Acht 
gelassen werden, da nur durch eine objektive Klärung die Patienten- und Rechtssicherheit gewährleistet und Personal von Vorwürfen entlastet werden kann (Preuß et al. 2006). Gleichzeitig bedeutet die arztstrafrechtlich durchgeführte Sektion Gewissheit für Angehörige und bietet die Grundlage für etwaige Schadensersatzund Schmerzensgeldansprüche.

\subsubsection{Weiterführende Untersuchungen}

Bei der Anordnungspraxis der weiterführenden forensischen Untersuchungen wurden ebenfalls Veränderungen festgestellt. So wurde im jüngeren Zeitraum häufiger eine Sektion ohne weiterführende Untersuchung durchgeführt. Entsprechend ergibt sich ein starker Rückgang der fünf häufigsten Kombinationen weiterführender Untersuchungen (1. Alkohologie/Toxikologie - 2. Alkohologie 3. Histologie - 4. Alkohologie/Histologie/Toxikologie - 5. Alkohologie/Histologie). Die zunehmende Gleichgültigkeit in Bezug auf eine umfassende Fallaufklärung kann auch den Anstieg (+29,4 \%) ungeklärter Todesursachen erklären, da die Staatsanwaltschaften zum einen nur an der Klärung von Todesfällen interessiert sind, in denen ein Fremdverschulden in Betracht kommt, zum anderen auch im Justizwesen ein Kosten- und Sparzwang spürbarer geworden ist (Röhl 2000).

\subsubsection{Schwangerschaftsabbrüche}

Todesfälle in Verbindung mit Schwangerschaftsabbrüchen waren rückläufig. Die in dieser Untersuchung erfassten Fälle waren „illegale“ Abtreibungsversuche mit tödlichem Verlauf. Seit Änderung zur Strafbarkeit des Schwangerschaftsabbruches (1974 und 1993) durch das Bundesverfassungsgericht entwickelte sich diesbezüglich eine zunehmende gesellschaftliche Akzeptanz. Ein entsprechender Indikationskatalog wurde definiert (Beck und Woppen 1998, Schwenzer 2010). Durch kostenfreie Beratungsstellen und die Möglichkeit, medizinisch fachgerechte Abtreibungen vorzunehmen, erübrigten sich die früher heimlich und illegal durchgeführten Eingriffe, die nicht selten aufgrund der Hygiene und des Fehlens von Fachpersonal bzw. apparativer Ausstattung tödlich verliefen. 


\subsection{Fazit}

Durch gesellschaftliche Veränderungen innerhalb eines 40-jährigen Zeitraumes wurden Rückgänge bei Verkehrsunfällen, Arbeitsunfällen und tödlich verlaufenden Schwangerschaftsabbrüchen festgestellt. Gleichzeitig war ein Panoramawandel im Bereich des Intoxikationsprofils mit einem deutlichen Anstieg erfasster Rauschgifttodesfälle bemerkbar. Hierbei sollte der hohe Stellenwert der Sektion (mit weiterführenden Untersuchungen) nicht außer Acht gelassen werden und eine höhere Sektionsfrequenz angestrebt werden, um mögliche Veränderungen des Sterbegeschehens, insbesondere im nicht natürlichen Todesursachenspektrum, präzise erfassen und die hierfür nötigen präventiven bzw. gesundheitspolitischen Maßnahmen umsetzen zu können. Die übrigen Entwicklungen lassen sich in Einklang bringen mit den Ergebnissen anderer Institute, wobei die staatsanwaltschaftliche Haltung und der Ermessensspielraum für die Fallaufklärung von wesentlicher Bedeutung sind. Bedenklich ist der Anstieg ungeklärter Todesfälle bei Verbesserung diagnostischer Möglichkeiten. Vorschläge zur Durchführung von Verwaltungssektionen wurden mehrfach ausgesprochen, es fehlt letztlich an dem Willen politischer Entscheidungsträger, diese umzusetzen. Die vorliegende Arbeit hebt die Notwendigkeit eines bundesweit einheitlichen Standards zur Erfassung forensischer Sektionsdaten hervor, um eine direkte Vergleichbarkeit der Ergebnisse zwischen den Instituten zu gewährleisten. Das Deutsche Forensische Sektionsregister (DFS) als multizentrische Studie ermöglicht die zentrale und einheitliche Erfassung forensischer Sektionsdaten. Durch statistische Auswertungen der Daten sind epidemiologische Entwicklungen feststellbar, die zur Entwicklung präventiver Strategien und der Qualitätssicherung im Gesundheitswesen beitragen können (Bratzke et al. 2004). 


\section{Zusammenfassung}

Durch Veränderungen in unserer Gesellschaft können sich potenziell Verschiebungen im Profil der forensisch fassbaren Obduktionsfälle ergeben. Es wurde eine retrospektive Auswertung der Sektionsprotokolle ( $n_{\text {ges }}=5.190$ ) des Göttinger Instituts für Rechtsmedizin vorgenommen, wobei zwei getrennte Jahrzehnte miteinander verglichen wurden (1969-1978, $\mathrm{n}_{1}=2.501$, und 1998-2007, $\left.\mathrm{n}_{2}=2.689\right)$. Den größten Anteil (95\%) machten gerichtliche Obduktionen aus, der Anteil der Verwaltungssektionen fiel um $94 \%$. Die nicht natürlichen Todesfälle waren mit $59 \%$ (-16\%), die natürlichen mit $33 \%$ (+30\%) und die ungeklärten mit $7 \%$ (+29 \%) vertreten. Die Geschlechterverteilung blieb nahezu unverändert ( $m=66 \%$; w= 34 \%). Das mittlere Sterbealter stieg um 9 Jahre von 37,8 auf 46,8 Jahre. In der Gruppe der natürlichen Todesart gab es starke Rückgänge bei respiratorischen Erkrankungen (-85 \%) und Infektionen (-46 \%). Zunahmen konnten bei den kardiovaskulären Erkrankungen (+70\%), den gastrointestinalen Erkrankungen (+127\%), den tödlichen Embolien (+157 \%) sowie den Tumorerkrankungen (+175\%) beobachtet werden. Unter den nicht natürlichen Todesursachen stiegen die Intoxikationsfälle um $38 \%$ an. Tödliche Vergiftungen mit Methadon, Amphetaminen und Kokain wurden nur im jüngeren Zeitraum beobachtet. Opiatintoxikationen stiegen deutlich an. Starke Rückgänge hingegen konnten bei Intoxikationen mit Zyaniden, E 605, Barbituraten sowie Hypnotika ermittelt werden. Der Anteil der Tötungsdelikte sank um 58 \%. Ebenfalls stark rückläufig waren Arbeitsunfälle (-59\%) sowie Verkehrsunfälle (-39 \%). Suizide nahmen um $35 \%$ zu. Ferner wurden mehr als viermal so viele Obduktionen aufgrund des Verdachts eines Behandlungsfehlers angeordnet, die jedoch nicht zu mehr bestätigten Fehlern führten. Die juristische Anordnungspraxis beschränkte sich im jüngeren Zeitraum häufiger nur auf die Obduktion (+60\%). Weitere histologische Untersuchungen (-74\%), Alkoholuntersuchungen (-54\%) sowie Kombinationen aus histologischer und Alkoholuntersuchung (-96\%) wurden hingegen seltener durchgeführt.

Durch die heutige Anordnungspraxis scheint sich eine zunehmende Gleichgültigkeit in Bezug auf eine umfassende Fallaufklärung entwickelt zu haben. 
Die Ergebnisse dieser Studie liefern für die jeweiligen Zeitabschnitte überwiegend Übereinstimmungen mit denen anderer Institute (Frankfurt, Magdeburg, Hamburg, München, Dresden), sind jedoch differenziert zu betrachten, da die Fallaufklärungsrate sehr stark mit der Anordnungspraxis und den Ermessensspielräumen einzelner Staatsanwaltschaften vergesellschaftet ist. Die in dieser Arbeit erhobenen Ergebnisse innerhalb eines 40-jährigen Zeitraumes erlauben Rückschlüsse auf die Wirkung von gesundheitspolitischen Gesetzesänderungen bzw. Maßnahmen und spiegeln diese im Obduktionsgut wider. Andererseits gibt es Entwicklungen innerhalb unseres Obduktionsguts, die eine Grundlage für Gesetzesänderungen (Verwaltungssektion, ungeklärte Todesfälle) darstellen und politisch umgesetzt werden sollten. Daher sind gerade vor dem Hintergrund schließender Institute sowie des zunehmenden Kosten- und Sparzwangs im Gesundheits- und Justizwesen Auswertungen forensischer Sektionsdaten als Teil einer möglichen bundesweiten multizentrischen Studie (Deutsches Forensisches Sektionsregister) anzusehen und können präventiv zum Schutz der Gesellschaft (z.B. unentdeckte Tötungsdelikte, Medikamentensicherheit, Dopingfälle, Drogentodesfälle, Behandlungsfehler, Verkehrssicherheit, unerkannte Gefahrenquellen etc.) und der Rechtssicherheit genutzt werden. 


\section{Literaturverzeichnis}

Alamuti N: Zur Phänomenologie der Obduktionen im Zentrum der Rechtsmedizin in Frankfurt a.M. (1981-1983). Med. Diss. Frankfurt am Main 1998

Ashworth TG (1991): Inaedequacy of death certification: proposal for change. J Clin Pathol $\underline{44}$, 265-268

Banaschak S, Eisenmenger W, Kuznik J, Brinkmann B (1998): Exhumierungen und kein Ende. Eine vergleichende Analyse. Arch Kriminol 202, 38-43

Bauer MT, Potratz D, Göller T, Wagner A, Schäfer R (1991): Qualitätskontrolle durch Autopsie. Wie häufig korrigiert der Obduktionsbefund die klinische Diagnose? DMW $\underline{116}, 801-807$

Baxendale S, Cross D, Johnston R (2012): A review of the evidence on the relationship between gender and adolescents' involvement in violent behavior. Aggress Violent Behav, 17, 297-310

Beck L, Woppen C (1998): Schwangerschaftsabbruch aus rechtlicher und ärztlicher Sicht. Gynäkologe 31, 297-303

Bennett S, Farrington DP, Huesmann LR (2005): Explaining gender differences in crime and violence: The importance of social cognitive skills. Aggress Violent Behav $\underline{10}, 263-288$

Berster DA: Beurteilung von Auswirkungen der Gesetzesänderung des NordrheinWestfälischen Bestattungsgesetzes vom 17.06.2003 auf die Effektivität des Leichenschauwesens durch kritische Überprüfung von Todesbescheinigungen in einem ländlichen Gebiet (Kreis Steinfurt). Med. Diss. Münster 2006 
Bratzke H, Parzeller M, Köster F (2004): Deutsches Forensisches Sektionsregister startet: Ein Beitrag zur Qualitätssicherung. Dtsch Arztebl, 101, A1258-1260

Brinkmann B, Püschel K (1991): Definition natürlicher, unnatürlicher, unklarer Tod. Todesursachenklärung: Derzeitige Praxis. MedR $\underline{5}, 233-238$

Brinkmann B, Banaschak S, Bratzke H, Cremer U, Drese G, Erfurt C, Giebe W, Lang C, Lange E, Peschel O, Philipp KP, Püschel K, Riße M, Tutsch-Bauer E, Vock R, Du Chesne A (1997a): Fehlleistungen bei der Leichenschau in der Bundesrepublik Deutschland. Ergebnisse einer multizentrischen Studie (I). Arch Kriminol 199, 1-12

Brinkmann B, Banaschak S, Bratzke H, Cremer U, Drese G, Erfurt C, Giebe W, Lang C, Lange E, Peschel O, Philipp KP, Püschel K, Riße M, Tutsch-Bauer E, Vock R, Du Chesne A (1997b): Fehlleistungen bei der Leichenschau in der Bundesrepublik Deutschland. Ergebnisse einer multizentrischen Studie (II). Arch Kriminol 199, 65-74

Brinkmann B, Du Chesne A, Vennemann B (2002): Aktuelle Daten zur Obduktionsfrequenz in Deutschland. Dtsch Med Wochenschr 127, 791-795

Bundesanstalt für Arbeitsschutz und Arbeitsmedizin (2009): Zeitreihen - Unfallgeschehen - Gesamtzahlen. Sicherheit und Gesundheit bei der Arbeit. http://www.baua.de/de/Publikationen/Fachbeitraege/Suga-2009.pdf (aufgerufen am 05.05.2013)

Bundesärztekammer (2005): Stellungnahme zur „Autopsie“ (Langfassung) http://www.bundesaerztekammer.de/fileadmin/user upload/downloads/AutLang.pdf (aufgerufen am 01.03.2013) 
Bundeskriminalamt - Rauschgiftkriminalität: Bundeslagebild 2010 - Tabellenanhang. Tab. 5.3

http://www.bka.de/nn 231632/SharedDocs/Downloads/DE/Publikationen/Jahresberic hteUndLagebilder/Rauschgiftkriminalitaet/2010RauschgiftJahreskurzlageTabellen,te $\underline{\text { mplateld=raw, property=publicationFile.pdf/2010RauschgiftJahreskurzlageTabellen.p }}$ df (aufgerufen am 10.09.2014)

Bundeskriminalamt - Polizeiliche Kriminalstatistik (1970)

http://www.bka.de/nn 229340/SharedDocs/Downloads/DE/Publikationen/Polizeiliche Kriminalstatistik/pksJahrbuecherBis2011/pks1970,templateld=raw,property=publicati onFile.pdf/pks1970.pdf (aufgerufen am 19.09.2014)

Bundeskriminalamt - Polizeiliche Kriminalstatistik (1975)

http://www.bka.de/nn 229340/SharedDocs/Downloads/DE/Publikationen/Polizeiliche Kriminalstatistik/pksJahrbuecherBis2011/pks1975,templateld=raw,property=publicati onFile.pdf/pks1975.pdf (aufgerufen am 19.09.2014)

Bundeskriminalamt - Polizeiliche Kriminalstatistik (1990)

http://www.bka.de/nn 229340/SharedDocs/Downloads/DE/Publikationen/Polizeiliche Kriminalstatistik/pksJahrbuecherBis2011/pks1990,templateld=raw,property=publicati onFile.pdf/pks1990.pdf (aufgerufen am 19.09.2014)

Bundeskriminalamt - Polizeiliche Kriminalstatistik (2002)

http://www.bka.de/nn 242508/SharedDocs/Downloads/DE/Publikationen/Polizeiliche Kriminalstatistik/pksJahrbuecherBis2011/pks2002,templateld=raw,property=publicati onFile.pdf/pks2002.pdf (aufgerufen am 22.09.2014)

Bundeskriminalamt - Polizeiliche Kriminalstatistik (2007)

http://www.bka.de/nn 229340/SharedDocs/Downloads/DE/Publikationen/Polizeiliche Kriminalstatistik/pksJahrbuecherBis2011/pks2007,templateld=raw, property=publicati onFile.pdf/pks2007.pdf (aufgerufen am 19.09.2014) 
Bundeskriminalamt - Polizeiliche Kriminalstatistik (2010)

http://www.bka.de/nn 229340/SharedDocs/Downloads/DE/Publikationen/Polizeiliche Kriminalstatistik/pksJahrbuecherBis2011/pks2010,templateld=raw,property=publicati onFile.pdf/pks2010.pdf (aufgerufen am 19.09.2014)

Bundeskriminalamt - Polizeiliche Kriminalstatistik (2014)

http://www.bka.de/SharedDocs/Downloads/DE/Publikationen/PolizeilicheKriminalstati stik/2014/pks2014Jahrbuch,templateld=raw,property=publicationFile.pdf/pks2014Jah rbuch.pdf

(aufgerufen am 07.08.2015)

Bundesministerium für Gesundheit - Drogenbeauftragte der Bundesregierung (2012): Drogen- und Suchtbericht

http://www.drogenbeauftragte.de/fileadmin/dateien-dba/Presse/Downloads/12-05-

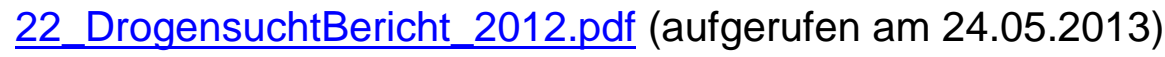

Casali MB, Mobilia F, Sordo SD, Blandino A, Genovese U (2014): The medical malpractice in Milan-Italy. A retrospective survey on 14 years of judicial autopsies. Forensic Sci Int 242, 38-43

Coradazzi AL, Morganti ALC, Montenegro MRG (2003): Discrepancies between clinical diagnoses and autopsy findings. Braz J Med Biol Res $\underline{36}, 385-391$

Dash SK, Behera BK, Patro S (2014): Accuracy in certification of cause of death in a tertiary care hospital-a retrospective analysis. J Forensic Leg Med 24, 33-6

Dettmeyer R, Madea B (1999): Rechtsmedizinische Gutachten im arztstrafrechtlichen Ermittlungsverfahren. MedR $\underline{12}, 533-539$

Dettmeyer R, Madea B (2002): Unsichere und uneinheitliche Rechtslage. Dtsch Arztebl 99, 2311-2314 
Du Chesne A, Bajanowksi T, Brinkmann B (1997): Spurenarme Tötungsdelikte an Kindern. Arch Kriminol 199, 21-26

Fernandez CC, La Harpe R (1996): Vorsätzliche Tötungsdelikte im Kanton Genf (1971 - 1990). Arch Kriminol 197, 111-117

Fischer J, Kleemann WJ, Tröger HD (1994): Types of trauma in cases of homicide. Forensic Sci Int $\underline{68}, 161-7$

Gatterburg A, Koischwitz C (2000): Verordnete Vertuschung. Der Spiegel, 37/2000 http://magazin.spiegel.de/EpubDelivery/spiegel/pdf/17322776 (aufgerufen am 05.05.2013)

Georgii A, Meliss RR (1992): Häufigkeit klinischer Obduktionen unter der Widerspruchs- gegenüber der Zustimmungslösung an der Medizinischen Hochschule Hannover. Pathologe $\underline{13}, 190-195$

Harbort S (2003): Das Hannibal-Syndrom. 9. Auflage; Piper Verlag, München

Hefer B, Wenning M (2006): Ärztliche Leichenschau und Todesbescheinigung. Folge 3. Rheinisches Arztebl $\underline{9}$, 15-17

Heinemann A, Ribbat J, Püschel K, Iwersen S, Schmoldt A (1998): Rauschgifttodesfälle mit Methadonbeteiligung (Hamburg 1990-1996). Rechtsmedizin $\underline{8}, 55-$ 60

Herden A: Zur Phänomenologie der Obduktionen im Zentrum für Rechtsmedizin in Frankfurt am Main 1999-2001. Med. Diss. Frankfurt 2008

Huusko R, Hirvonen J (1988): The problem of determining the manner of death as suicide or accident in borderline cases. Rechtsmedizin $\underline{100}, 207-213$ 
Jäger A: Statistische Analyse der Obduktionen dreier rechtsmedizinischer Institute Berlins der Jahre 1999-2003. Med. Diss. Berlin 2008

Kausche B: Statistische Analyse der Obduktionen des Institutes für Rechtsmedizin Magdeburg 1985-1995. Med. Diss. Magdeburg 1998

Killias M (1993): International correlations between gun ownership and rates of homicide and suicide. Can Med Assoc J 148, 1721-1725

Knobloch HG: Zur Phänomenologie der Obduktionen im Zentrum der Rechtsmedizin in Frankfurt am Main 1978-1980. Med. Diss. Frankfurt 1996

Koch G: Analyse von Todesbescheinigungen in einem ländlich abgegrenzten Gebiet in der Peripherie eines rechtsmedizinischen Einzugsbereiches im Kreis Lippe. Med. Diss. Münster 2004

Larsen ST, Lynnerup N (2011): Medico-legal autopsies in Denmark. Dan Med Bull $\underline{58}, 1-4$

Latsch G, Meyer C (2005): Tote klagen nicht. Der Spiegel, Ausgabe 37/2005 http://magazin.spiegel.de/EpubDelivery/spiegel/pdf/41768188 (aufgerufen am 05.06.2013)

Madea B: Die ärztliche Leichenschau. Rechtsgrundlagen, Praktische Durchführung, Problemlösung. 2. Auflage; Springer Verlag, Heidelberg 2006

Madea B: Praxis Rechtsmedizin. Befunderhebung Rekonstruktion Begutachtung. 2. Auflage; Springer, Berlin Heidelberg 2007

Madea B, Dettmeyer R: Basiswissen Rechtsmedizin. Springer, Heidelberg 2007 
Madea B, Püschel K, Lignitz E, Dettmeyer R (2006a): Verwaltungssektionen - Inhalt, Zweck, Notwendigkeit, gesetzliche Regelungen. Rechtsmedizin 16, 13-22

Madea B, Püschel K, Lignitz E, Dettmeyer R (2006b): Verwaltungssektion. Dringende Notwendigkeit. Dtsch Arztebl 103, A 914-918

Madea B, Dettmeyer R, Schmidt P: Sektionsrecht; in: Madea B (Hrsg.): Praxis Rechtsmedizin - Befunderhebung, Rekonstruktion, Begutachtung. 2. Auflage; Springer, Heidelberg 2007, 65-69

Modelmog D: Todesursachen sowie Häufigkeit pathologisch-anatomischer Befundkomplexe und Diagnosen in einer mittelgroßen Stadt bei fast 100\%iger Obduktionsquote. Deutsche Hochschulschriften 491. Hänsel-Hohenhausen, Egelsbach 1993

Moschkau N, Kunz S, Fieseler S, Graw M, Zinka B (2011): Sektionsdaten des Instituts für Rechtsmedizin der Universität München aus dem Jahr 2003. Rechtsmedizin 21, 541-548

Nestler K, Gradistanac C, Wittekind C (2008): Evaluation des klinischen Nutzen der Obduktion. Pathologe $\underline{29}$, 449-454

Nick MB: Todesumstände und Todesursachen der Bevölkerung der Gemeinden des Landkreises Harburg/Niedersachen. Med. Diss. Hamburg 2006

Niederländer E (2006): „Die Todesursachen in der EU“ in Statistik kurz gefasst Bevölkerung und soziale Bedingungen.

http://ec.europa.eu/eurostat/documents/3433488/5440977/KS-NK-06-010-DE.PDF (aufgerufen am 06.07.2013)

Nordrum I, Eide TJ, Jørgensen L (1998): Medicolegal Autopsies of violent deaths in northern Norway 1972-1992. Forensic Sci Int 92, 39-48 
Oehme C: Statistisch-deskriptive Auswertung der Sektionsdaten des Instituts für Rechtsmedizin der Universität München aus dem 2007. Med. Diss. München 2012

Opitz T: Zur Phänomenologie der Obduktionen im Institut für Rechtsmedizin der TU Dresden (1998 - 2003). Med. Diss. Dresden 2012

Preuß J, Dettmeyer R, Madea B (2006): Begutachtung behaupteter letaler Behandlungsfehler im Fach Rechtsmedizin. Rechtsmedizin 16, 367-382

Price JH, Everett SA, Bedell AW, Telljohann SK (1997): Reduction of firearm-related violence through firearm safety counseling. The role of family physicians. Arch Fam Med $\underline{6}, 79-83$

Projektstudie über das Mobilitätsverhalten von Serien- und Intensivtätern. Durchgeführt von der Fachhochschule der Polizei. Wiesbaden 1996

Püschel K (2009): Quo vadis „ärztliche Leichenschau“? Rechtsmedizin 19, 389-390

Ravakhah K (2006): Death certificates are not reliable: Revivification of the autopsy. South Med J $\underline{99}$, 728-733.

Razum O, Breckenkamp J (2007): Kindersterblichkeit und soziale Situation: Ein internationaler Vergleich. Dtsch Arztebl 104, A2950-2956

Riemann J: Zur Phänomenologie der Obduktionen im Zentrum für Rechtsmedizin in Frankfurt am Main 1993-1995. Med. Diss. Frankfurt 2003

Röhl KF (2000): Justiz als Wirtschaftsunternehmen. Budgetierung, Controlling und Professionalisierung der Justizverwaltung. Deutsche Richterzeitung, 220-230

Rothschild MA (2009): Probleme bei der ärztlichen Leichenschau. Rechtsmedizin $\underline{19}$, 407-412 
Rückert S (2000): Sparen mit tödlichen Folgen. Zeit Online Wissen, Ausgabe $20 / 2000$

http://pdf.zeit.de/2000/20/200020.kriminalitaet .xml.pdf (aufgerufen 13.07.2013)

Rückert S: Tote haben keine Lobby. 1. Auflage; Ullstein Verlag, München 2002

Rückert S (2003): Mord an der Rechtsmedizin. Zeit Online Wissen, Ausgabe 47/2003 http://pdf.zeit.de/2003/47/Rechtsmedizin-Inst .pdf (aufgerufen am 27.07.2013)

Scheib, K: Die Dunkelziffer bei Tötungsdelikten aus kriminologischer und rechtsmedizinischer Sicht. Jur. Diss. Berlin 2002

Schieche C, Schmeling A, Strauch H, Geserick G (2000): Tödliche Arbeitsunfälle in Berlin von 1990-1995 aus rechtsmedizinischer Perspektive. Rechtsmedizin 10, 138143

Schmidtke A, Sell R, Löhr C, Gajewska A, Schaller S (2009): Epidemiologie und Demographie des Alterssuizids. Suizidprophylaxe $\underline{36}, 12-20$

Schulz Y: Statistisch-deskriptive Auswertung des Obduktionsgutes des Institutes für Rechtsmedizin der Medizinischen Hochschule Hannover der Jahre 1978-1982 sowie 1994-1998. Med. Diss. Hannover 2007

Schwarze EW (2001): Die Bedeutung der Autopsie - heute und morgen. Verh Dtsch Ges Path $\underline{85}, 118-131$

Schwarze EW, Pawlitschko J (2003): Autopsie in Deutschland. Derzeitiger Stand, Gründe für den Rückgang der Obduktionszahlen und deren Folgen. Dt. Ärzteblatt $\underline{100}$, A2802-2808

Schwenzer T (2010): Schwangerschaftsabbruch. Gynäkologe 43, 35-43 
Sellin F, Weber K: Todesursache: natürlich. Warum die meisten Morde unentdeckt bleiben. Rowohlt Verlag, Reinbek 2001

Simonsen KW, Hansen C, Rollmann D, Kringsholm B, Breum Müller I, Johansen SS, Linnet K (2011): Drug-related death in Denmark in 2007. Danish medical bulletin $\underline{58}$, A4307

Sperhake J, Püschel K (2003): Das Hamburger Sektionsgesetz vom 09. Februar 2000 - Entwicklung der Sektionszahlen in Hamburgs Prosekturen. Pathologe 24, 204-206

Statistisches Bundesamt (2011): Unfallentwicklung auf deutschen Straßen 2010 https://www.destatis.de/DE/PresseService/Presse/Pressekonferenzen/2011/Unfaelle 2010/pressebroschuere unfaelle.pdf? blob=publicationFile (aufgerufen am 10.07.2013)

Statistisches Bundesamt (2013): Todesursachen in Deutschland 2012 https://www.destatis.de/DE/Publikationen/Thematisch/Gesundheit/Todesursachen/To desursachen2120400127004.pdf? blob=publicationFile

(aufgerufen am 20.12.2013)

Thomsen H, Schewe G (1994): Ärztliche Leichenschau. Probleme im ärztlichen Bereich, bei Ermittlungsbehörden und bei landesrechtlichen Regelungen. Arch Kriminol 193, 79-89

Vennemann B, Du Chesne A, Brinkmann B (2001): Die Praxis der ärztlichen Leichenschau. Dtsch Med Wochenschr $\underline{126}, 712-716$

Vennemann M, Berger K, Richter D, Baune BT (2006): Unterschätzte Suizidraten durch unterschiedliche Erfassung in Gesundheitsämtern. Dtsch Arztebl, 103, 12221226 
Vock R, Trauth W, Bonte W, Betz P, Gerling I, Graw M, Hartge K, Hilgermann R, Höhmann E, Kampmann H et al. (1999): Tödliche Kindesmisshandlung (durch physische Gewalteinwirkung) in der Bundesrepublik Deutschland im Zeitraum 1.1.1985 bis 2.10.1990. Arch Kriminol 203, 73-85

Wirth I, Strauch H: Rechtsmedizin - Grundwissen für die Ermittlungspraxis. Kriminalistik Verlag, Heidelberg 2006, 58-59 


\section{Abkürzungsverzeichnis}

Alk.

allg.

Auß.sekt.

BRD

$\mathrm{CO}$

$\mathrm{CoHb}$

DDR

Histo.

JVA

k.A.

Krhs.

Lkw

Psych.

Neuropath.

NRW

posttraumat.

Pkw

SIDS

StPO

sonst.

THC

Tox.

V.a.

ZNS
Alkohologie

allgemein

Außensektion

Bundesrepublik Deutschland

Kohlenmonoxid

Carboxyhämoglobin

Deutsche Demokratische Republik

Histologie

Justizvollzugsanstalt

keine Angabe

Krankenhaus

Lastkraftwagen

Psychiatrie

Neuropathologie

Nordrhein-Westfalen

posttraumatisch

Personenkraftwagen

Sudden Infant Death Syndrome (plötzlicher Kindstod)

Strafprozessordnung

sonstige

Tetrahydrocannabinol

Toxikologie

Verdacht auf

zentrales Nervensystem 


\section{Datenerhebungsbogen}

\section{Basisdaten}

\subsection{ID}

Die ID ist eine vom Computerprogramm für jeden Datensatz automatisch vergebene Nummer, die von 0 aufwärts entsprechend der Anzahl der Fälle gezählt wird.

\subsection{Laufende Nummer}

Eine vom Institut vergebene laufende Nummer für jeden Fall.

\subsection{Sektionsjahr}

$x x x=$ Angabe in Jahren

\subsection{Sektionsmonat}
1 = Januar
$7=$ Juli
2 = Februar
$8=$ August
3 = März
9 = September
$4=$ April
$10=$ Oktober
$5=$ Mai
$11=$ November
$6=$ Juni
$12=$ Dezember

\subsection{Sektionsart}

1 = gerichtliche Sektion

2 = klinische-/Verwaltungssektion

3 = sonstige (Privat-, Versicherungs- und berufsgenossenschaftliche) Sektion 


\subsection{Sektionsort}

$0=$ unbekannt/fehlende Angabe

4 = Außensektion Bremen

1 = Göttingen

$5=$ Außensektion Nordrhein-Westfalen

$2=$ Außensektion Niedersachsen

$6=$ sonstige Außensektion

3 = Außensektion Hessen

\subsection{Sterbe- /Auffindeort}

$1=$ Wohnung

$5=$ Krankenhaus/Psychiatrie/Pflegeheim

2 = Öffentlichkeit

$6=$ Polizeigewahrsam $/$ JVA

$3=$ Straßenverkehr

7 = unbekannt/Sonstiges

$4=$ Arbeit

\subsection{Leichenidentität}

$0=$ unbekannt

1 = bekannt

2 = primär unbekannt \& später identifiziert

\subsection{Geburtsjahr}

$\operatorname{xxxx}=$ Jahr

$0=$ unbekannt

\subsection{Alter}

$x x=$ Lebensalter in Jahren

$0=$ unbekannt

\subsection{Geschlecht}

1 = männlich

$2=$ weiblich

\subsection{Leichenfundortbesichtigung}

$0=$ keine Besichtigung

$1=$ Besichtigung 


\subsection{Intervall zwischen Leichenfund und Obduktion}

$x x=$ Angabe in Tagen

$0=$ unbekannt bzw. nicht seriös abschätzbar

\subsection{Postmortale Gesamtliegezeit}

$x x=$ Angabe in Tagen

\subsection{Leichenzustand}

$0=$ unbekannt $/$ keine Angabe

3 = mittelgradige Fäulnis

$1=$ frisch

$4=$ Madenbefall/starke Fäulnis

2 = leichte Fäulnis

$5=($ Teil-)Skelettierung

\subsection{Todesart vor Obduktion}

$1=$ natürlich

3 = nicht geklärt

2 = nicht natürlich

\subsection{Todesart nach Obduktion}

$1=$ natürlich

3 = nicht geklärt

2 = nicht natürlich

\subsection{Todesart: Kategorie nach Obduktion}

$0=$ nicht angegeben

$5=$ Suizid

1 = natürlich

$6=$ Tötung

$2=$ Verkehrsunfall

$7=$ Intoxikation

$3=$ Arbeitsunfall

8 = Vernachlässigung

4 = sonstiger Unfall

$9=$ Sonstiges 


\subsection{Besonderheiten/Todesumstände}

$0=$ keine Besonderheiten

$8=$ Mehrleichenfund

$1=$ Sexualdelikt

$9=$ Verdacht auf erweiterten Suizid

$2=$ Drogentodesfall

$10=$ Verdacht auf SIDS

$3=$ Exhumierung

11 = autoerotischer Unfall

$4=$ Nachsektion

$12=$ Wasserleiche

$5=$ Mors in actu

$13=$ Kindesmisshandlung

$6=$ Schwangerschaft

$14=$ Brandleiche

7 = Schwangerschaftsabbruch

$15=$ sonstige

\subsection{Behandlungsfehler}

$0=$ nicht zutreffend

2 = bestätigt

$1=$ verneint

3 = nicht geklärt

\subsection{Evidenzgrad der Todesursache}

$0=$ nicht zutreffend

$2=$ Gruppe 2

$1=$ Gruppe 1

$3=$ Gruppe 3

\subsection{Sterbenstyp bei natürlichem Tod}

1 = linearer Sterbenstyp

2 = divergierender Sterbenstyp
3 = konvergierender Sterbenstyp

4 = komplexer Sterbenstyp

\subsection{Basis der Todesursachenklärung (weiterführende Untersuchungen)}

$1=$ Obd

2 = Obd. + Alkohol

$3=$ Obd. + Alkohol + Toxikologie

4 = Obd. + Toxikologie

$5=$ Obd. + Histologie

$6=$ Obd. + Histologie + Toxikologie

$7=$ Obd. + Mikrobiologie 
8 = Obd. + Mikrobiologie + Histologie

$9=$ Obd. + Mikrobiologie + Histologie + Toxikologie

$10=$ Sonstige Kombinationen

$11=$ Obd. + Histologie + Alkohol

12 = Obd. + Alkohol + Histologie + Toxikologie

13 = Obd.+ Histologie + Mikrobiologie + Neuropathologie

14 = Obd. + Histologie + Alkohol + Toxikologie + Neuropathologie

$15=$ Obd.+ Histologie + Neuropathologie

$16=$ Neuropathologie

$17=$ Sonstiges

\subsection{Pflegezustand}

$1=$ unauffällig

$5=$ Dekubitus III $^{\circ}$

2 = Zeichen für Vernachlässigung

$6=$ Dekubitus $\mathrm{IV}^{\circ}$

$3=$ Dekubitus $^{\circ}$

7 = nicht beurteilbar/nicht kommentiert

$4=$ Dekubitus $\|^{\circ}$

\section{Natürliche Todesursachen}

\subsection{Respirationstrakt}

$1=$ Pneumonie

$2=$ Pneumonie mit Komplikation

$3=$ akute Bronchitits

4 = COLD-Emphysem

$5=$ Asthma-Anfall
$6=$ Pneumothorax

7 = Lungenarterienthrombembolie

$8=$ Bronchitis

$9=$ Pneumonie + Bronchitis

$10=$ Sonstiges 


\subsection{Herz-Kreislauf-System}

1 = akute Koronarinsuffizienz bei stenos. Koronarsklerose

2 = akute Koronarinsuffizienz bei Koronarthrombose

$3=$ akuter Herzinfarkt

4 = alter Herzinfarkt (Re-Infarkt)

$5=$ Herzbeuteltamponade bei Herzinfarkt

$6=$ Herzbeuteltamponade bei Aortenruptur

$7=$ Herzhypertrophie (normal: 4-5 Promille des KG)

8 = Herzhypertrophie über $500 \mathrm{~g}$

$9=$ Kardiomyopathie

$10=$ angeborene Herzfehler

$11=$ Myokarditis

$12=$ Cor pulmonale

$13=$ Aortenaneurysma

$14=$ Gefäßanomalie

$15=$ Klappenerkrankung

$16=$ Herzversagen (allgemein)

$17=$ Gefäßerkrankung allgemein

$18=$ Herz-Kreislauf-Versagen

$19=$ Sonstiges

\subsection{Zentrales Nervensystem}

$1=$ Hirnblutung

$2=$ Hirninfarkt

3 = Hirnödem

$4=$ intravitaler Hirntod/hypox. Hirnschaden

$5=$ Enzephalitis

$6=$ Meningitis
$7=$ Status epilepticus

8 = subdurales Hämatom

$9=$ subarachnoidale Blutung

$10=$ Hirngefäßerkrankung

$11=$ Sonstiges 


\subsection{Gastrointestinaltrakt}

$1=$ Peritonitis

$2=$ Ileus

$3=$ Gastroenteritis

$4=$ Enterokolitis

$5=$ Dyspepsie/ Dystrophie (Kinder)

$6=$ Coma hepaticum

\subsection{Urogenitaltrakt}

$1=$ Urämie

2 = Pyelonephritis

\subsection{Endokrines System}

1 = Coma diabeticum

2 = Hypoglykämie

$3=$ thyreotoxische Krise

\subsection{Tödliche Embolie}

$1=$ Thrombembolie

$2=$ Fettembolie

\subsection{Verbluten}

1 = Hämoptoe

$2=$ Melaena

3 = Hämatothorax

4 = Hämaskos

\subsection{Infekte}

$1=$ Sepsis

2 = Grippe
$7=$ akute Pankreatitis

$8=$ Ösophagusvarizenruptur

$9=$ Organperforation

$10=$ Magenulkus

$11=$ Sonstiges

$3=$ Sonstiges

4 = hypertensive Krise

$5=$ Sonstiges

3 = Luftembolie

$4=$ Sonstiges

$5=$ Verbluten (allg.) nach innen

$6=$ Gerinnungsstörung $/$ Koagulopathie

$7=$ Sonstiges

3 = polylokale Infekte

$4=$ Sonstiges 


\subsection{Stoffwechselentgleisungen}

1 = Alkoholentzugsdelir

$2=$ Anorexia nervosa

\subsection{Tumoren}

$1=$ Tumorkachexie

2 = Herz-Kreislauf-Versagen

\subsection{Fruchttod}

1 = intrauterine Asphyxie

2 = Fruchtwasseraspiration

$3=$ Missbildung

\subsection{SIDS}

$1=\operatorname{SIDS}$
3 = Stoffwechselentgleisung (allg.)

$4=$ Sonstiges

$3=$ lokale Komplikation

$4=$ Sonstiges

4 = Dystrophie

$5=$ Sonstiges

\section{Nicht natürliche Todesursachen}

\subsection{Gewalteinwirkungen}

\subsubsection{Stumpfe Gewalt}

1 = Sturz

$3=$ Schlag $/$ Tritt

$2=$ Verkehrsunfall

$4=$ Sonstiges

\subsubsection{Scharfe Gewalt}

1 = Schnitt

$3=$ Schnitt \& Stich

$2=$ Stich 


\subsubsection{Halbscharfe Gewalt/Hiebverletzung}

$1=$ Hieb

\subsubsection{Schuss}

$1=$ Steckschuss

$2=$ Durchschuss

\subsubsection{Tatwaffe (Schuss- bzw. Explosionseinwirkung)}

$1=$ Pistole

$2=$ Revolver

$3=$ Gewehr

\subsubsection{Strangulation}

$1=$ Erhängen (typisch)

2 = Erhängen (atypisch)

$3=$ Erdrosseln

4 = Erwürgen

\subsubsection{Ersticken}

$1=$ Aspiration

$2=$ Verschluss der Atemwege

$3=$ Thoraxkompression

\subsubsection{Ertrinken/Tod im Wasser}

$1=$ Tod nach Beinahe-Ertrinken

$4=$ Tauchunfall

2 = sog. Badetod

$5=$ Sonstiges

$8=$ Sonstiges

4 = Plastiktüte

$5=$ Sonstiges
3 = mutmaßliches Ertrinken

$5=$ Halskompression (allgemein)

$6=$ Kombinationen

$7=$ Erhängen (allgemein)

\subsubsection{Physikalische Noxen}

[Freitext] 


\subsubsection{Chemische Noxen}

1 = Säure

$2=$ Lauge

\subsubsection{Thermische Schädigung}

1 = Hitze

$2=$ Kälte

\subsubsection{Elektrizität}

1 = Niederspannung

3 = systemische Stromeinwirkung

$2=$ Hochspannung

$4=$ Blitzschlag

\subsubsection{Intoxikation}

$1=$ Alkohol

$7=E 605$

$2=$ Mischintoxikation

$8=$ Barbiturate

$3=$ Morphin $/$ Heroin

9 = Hypnotika allgemein

$4=$ Methadon

$10=$ Zyanid

$5=$ Kokain

$11=\mathrm{CO}$

$6=$ Amphetamin

$12=$ sonstige Intoxikation

\subsubsection{Verhungern/Verdursten}

1 = Verhungern/Verdursten

2 = Sonstiges

\subsubsection{Sonstige Einwirkung}

$1=$ Bolustod 


\subsection{Todesumstände}

\subsubsection{Verkehrsunfall}
1 = Fußgänger
$6=$ Fahrradfahrer
$2=$ Pkw-Fahrer
$7=$ Schienenfahrzeug
$3=$ Lkw-Fahrer
8 = Flugzeug
$4=$ Pkw-Insasse/Beifahrer
$9=$ Sonstiges
$5=$ Kraftradfahrer

\subsubsection{Tod nach medizinischen Maßnahmen}

$1=$ Tod nach Medikation

4 = Tod nach Operation oder Eingriff

$2=$ Narkosezwischenfall

$5=$ Sonstiges

$3=$ Mors in tabula

$\mathrm{x}=$ Fachrichtung (Angabe im Freitext)

\subsubsection{Neugeborene/Foeten}

1 = Gelebthaben bewiesen

$2=$ Gelebthaben nicht bewiesen oder Totgeburt

3 = Schwangerschaftsabbruch

$4=$ Sonstiges

\subsubsection{Tatwerkzeuge (stumpflscharf)}

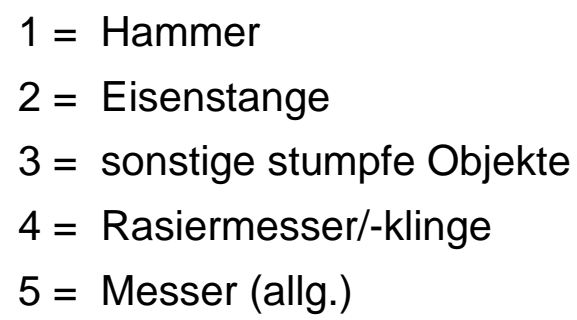

$6=$ Axt $/$ Beil

$7=$ Trokar

$8=$ Flasche/Glas

$9=$ Dolch

$10=$ sonstige scharfe Objekte 


\subsubsection{Systemische bzw. direkte Todesursachen}

$1=$ Verbluten nach innen

$2=$ Verbluten nach außen

$3=$ Verbluten nach innen und außen

4 = Herzbeuteltamponade

$5=$ zentrales Regulationsversagen
$6=$ Polytrauma

7 = Organruptur(en)

$8=$ Pneumothorax

$9=$ Kombination

$10=$ Sonstiges

$6=$ Sepsis

$7=$ Kombinationen

8 = Luftembolie

$9=$ Anaphylaxie

$10=$ Sonstiges

4. Quantitative und qualitative Toxikologie-IAlkoholbefunde

\section{Freitext}




\section{Danksagung}

Ich möchte mich herzlich bei allen bedanken, die zu der Entstehung dieser Arbeit beigetragen haben oder mich auf dem Wege unterstützt haben.

Allen voran danke ich meinem Doktorvater und Betreuer Herrn Prof. Dr. W. Grellner für die Überlassung der interessanten Fragestellung und die Möglichkeit, meine Doktorarbeit durchführen zu dürfen.

Ebenso bedanken möchte ich mich bei allen Mitarbeitern des Instituts für Rechtsmedizin, die mich während dieser Zeit helfend unterstützt haben.

Außerdem gebührt den Mitarbeitern des Instituts für Medizinische Statistik (David Ellenberger, Hans Joachim Helms) und der Medizinischen Informatik (Prof. U. Sax) ein Dankeschön für die freundliche Unterstützung bei der Erstellung der Datenbank und der statistischen Auswertung, die dieser Arbeit zugrunde liegen. 


\section{Lebenslauf}

Aus Gründen des Datenschutzes wird bei der Online-Publikation auf den Abdruck des Lebenslaufes verzichtet. 